\title{
Host relationships and geographic distribution of species of Acanthobothrium Blanchard, 1848 (Onchoproteocephalidea, Onchobothriidae) in elasmobranchs: a metadata analysis
}

\author{
Francisco Zaragoza-Tapia', Griselda Pulido-Flores ${ }^{1,2}$, \\ Scott L. Gardner², Scott Monks ${ }^{1,2}$ \\ I Universidad Autónoma del Estado de Hidalgo, Centro de Investigaciones Biológicas, Apartado Postal 1-10, \\ C.P. 42001, Pachuca, Hidalgo, México 2 Harold W. Manter Laboratory of Parasitology, University of Nebras- \\ ka-Lincoln, Lincoln, NE 68588-0514, USA \\ Corresponding author: Scott Monks (monks.scott@gmail.com) \\ Academic editor: Boyko Georgiev | Received 6 September 2019 | Accepted 7 April 2020 | Published 11 June 2020 \\ http://zoobank.org/95F2582D-A68C-4728-868D-EEDD5D97B7ED \\ Citation: Zaragoza-Tapia F, Pulido-Flores G, Gardner SL, Monks S (2020) Host relationships and geographic \\ distribution of species of Acanthobothrium Blanchard, 1848 (Onchoproteocephalidea, Onchobothriidae) in \\ elasmobranchs: a metadata analysis. ZooKeys 940: 1-49. https://doi.org/10.3897/zookeys.940.46352
}

\begin{abstract}
Species of Acanthobothrium have been documented as parasites of the spiral intestine of elasmobranchs. Results of a metadata analysis indicate that 114 species of elasmobranchs have been reported as hosts of 200 species of Acanthobothrium. The metadata analysis revealed that $3.7 \%$ of species of sharks and $14.9 \%$ of species of rays that have been reported as hosts to date; some species are parasitized by more than one species of Acanthobothrium. This work provides a Category designation, as proposed by Ghoshroy and Caira (2001), for each species of Acanthobothrium. These Category designations are a tool to facilitate comparisons among members of Acanthobothrium for descriptions of new species in the future.
\end{abstract}

\section{Keywords}

Biodiversity, Elasmobranchii, Eucestoda, geographic distribution, rays, sharks

Copyright Francisco Zaragoza-Tapia et al. This is an open access article distributed under the terms of the Creative Commons Attribution License (CC BY 4.0), which permits unrestricted use, distribution, and reproduction in any medium, provided the original author and source are credited. 


\section{Introduction}

According to Last et al. (2016b), there are 34 families comprised of 516 valid species of sharks and 26 families that include 633 valid species of rays. Since that publication, six new species of sharks and rays were described by: Yokota and Carvalho (2017) (two species of rays), Vaz and Carvalho (2018) (one species of shark), Rutledge (2019) (one species of ray), Grace et al. (2019) (one species of shark) and Concha et al. (2019) (one species of ray). This brought the current number of recognized species to 517 species of sharks and 637 species of rays.

Elasmobranchs (sharks, skates and rays) are host to a great variety of parasites in nature, particularly helminths. Acanthobothrium Blanchard, 1848 (Onchoproteocephalidea) is the most diverse genus that has been reported as parasite of the spiral intestine of elasmobranchs (Caira and Jensen 2017). At the present time, 201 species of Acanthobothrium are considered to be valid (Maleki et al. 2013; Caira and Jensen 2017; Rodríguez-Ibarra et al. 2018; Franzese and Ivanov 2018; Maleki et al. 2019; Zaragoza-Tapia et al. 2019, 2020). The genus consists of species that exclusively parasitize elasmobranchs as adults and, in many cases, individual species are thought to parasitize only a single species of elasmobranch (Caira 2011; Caira and Jensen 2017). Therefore, the genus Acanthobothrium is an excellent model for future studies of hostparasite co-speciation.

The main goal of this work is to provide a revised checklist based on a metadata analysis of the host relationships of members of Acanthobothrium and their geographic distribution based on records that have been generated from different parts of the world. The checklist focuses on the 201 valid species of Acanthobothrium and reports correlated with the genera and species of elasmobranchs, and includes the geographical distribution of each.

The number of species of Acanthobothrium continues to grow and there are still regions of the world without a single report of this genus (see Figure 1). For some time, the process of distinguishing new species of Acanthobothrium from existing species has become more and more unwieldy because of the large number of species. As an identification tool, Ghoshroy and Caira (2001) developed a categorical method for identifying species for initial comparisons. Therefore, in order to provide an update to this method, categorical designations are provided in the present checklist for each species of Acanthobothrium in the manner proposed by Ghoshroy and Caira (2001). The categories are based on and obtained from the combination of four quantitative characters: total length of the worm; the number of proglottids comprising the strobila; the number of testes per proglottid; and symmetry of the ovarian lobes. This categorical designation allows parasitologists working with this genus to postulate a group of similar species, those of the same category designation, for comparison of a new species or to aid in the preliminary identification of known species. As an additional aid, in the checklist the accession number, if known, of type specimens of each species is provided. 


\section{Materials and methods}

The checklist, updated until March 2020, was based on bibliographical information from two sources of information: 1. a compilation of the records of species of Acanthobothrium as originally described, complemented by information gathered from Global Cestode Database (Caira et al. 2019) and from recent compilation studies (e.g., Ghoshroy and Caira 2001; Campbell and Beveridge 2002; Fyler and Caira 2006; Caira and Jensen 2017); and 2. information for the distribution and taxonomy of elasmobranchs that integrated a bibliographical search using different databases of literature published to date (e.g., Del Moral-Flores et al. 2015; Last et al. 2016b; Merlo-Serna and GarcíaPrieto 2016; Alves et al. 2017) and data from FishBase (Froese and Pauly 2019).

In the checklist, the species of Acanthobothrium are arranged in alphabetical order. The scientific names and geographic distribution of elasmobranchs have been updated based on Last et al. (2016a, 2016b), Amaral et al. (2018) and Froese and Pauly (2019). The regional classification scheme of the geographic distribution of the hosts is according to Last et al. (2016b) with additional information from Froese and Pauly (2019). The following abbreviations are used for biogeographic regions (see Figure 1):

$\begin{array}{llll}\text { ARC } & \text { Arctic Ocean; } & \text { NIO } & \text { Northern Indian Ocean; } \\ \text { ECA } & \text { Eastern Central Atlantic; } & \text { SOC } & \text { Southern (Antarctic) Ocean; } \\ \text { ECP } & \text { Eastern Central Pacific; } & \text { WCA } & \text { Western Central Atlantic; } \\ \text { EIO } & \text { Eastern Indian Ocean; } & \text { WCP } & \text { Western Central Pacific; } \\ \text { ENA } & \text { Eastern North Atlantic; } & \text { WIO } & \text { Western Indian Ocean; } \\ \text { ENP } & \text { Eastern North Pacific; } & \text { WNA } & \text { Western North Atlantic; } \\ \text { ESA } & \text { Eastern South Atlantic; } & \text { WNP } & \text { Western North Pacific; } \\ \text { ESP } & \text { Eastern South Pacific; } & \text { WSA } & \text { Western South Atlantic; } \\ \text { MED } & \text { Mediterranean Sea; } & \text { WSP } & \text { Western South Pacific. }\end{array}$

Information for each species of Acanthobothrium presented herein includes the name of the species, authority (original description referenced in the literature cited), abbreviation of the name of the collection where specimens are deposited and the accession numbers of the specimens, followed by the status of the specimens (holotype, paratype, neotype, syntype or voucher). The acronym "NR" was used for data that are not reported in the original source. Localities (type or/and additional localities) were given and referenced in the literature cited. A Category designation was supplied for all species using the categorical method proposed by Ghoshroy and Caira (2001).

The categorical method was developed as a system of grouping species of Acanthobothrium based on the combination of four qualitative characters: the total length of worms- $\leq 15 \mathrm{~mm}=\mathrm{S}$ (short) or $>15 \mathrm{~mm}=\mathrm{L}$ (long); the number of proglottids comprising the strobila- $\leq 50=\mathrm{F}$ (few) or $>50=\mathrm{M}$ (many); the number of testes per proglottid- $\leq 80=\mathrm{F}$ (few) or $>80=\mathrm{M}$ (many); and symmetry of the ovarian lobessymmetrical $=\mathrm{S}$ or asymmetrical $=\mathrm{A}$. Of the possible combinations the following 10 


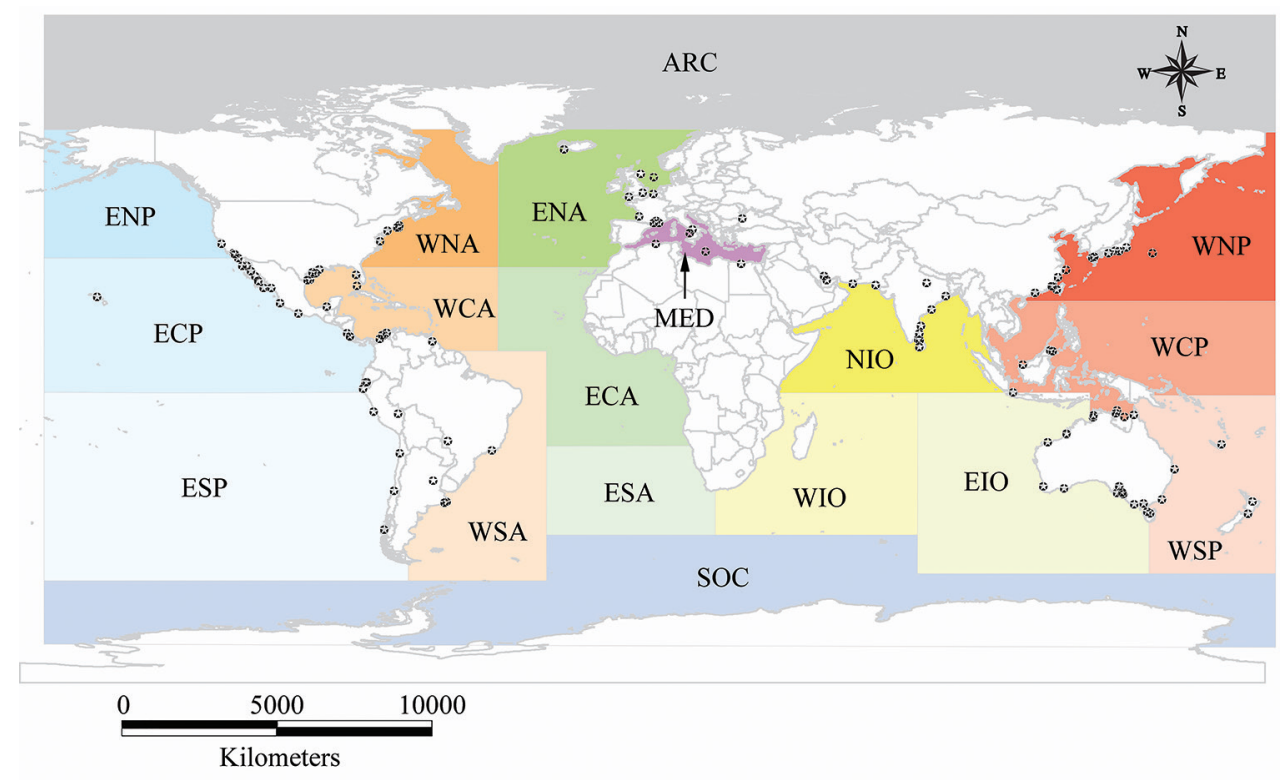

Figure I. Type localities of species of Acanthobothrium reported worldwide and the biogeographic regions (Last et al. 2016b) of the geographic distribution of their hosts (see Table 1).

categories currently are recognized and coded as follows: $1=$ SFFS; $2=$ SFFA; $3=$ LMMA; 4 = LMMS; 5 = LMFS; 6 = LMFA; 7 = LFFA; 8 = SMFS; $9=$ LFFS; $10=$ SMMS. This method limited the number of necessary comparisons required in the description between known species with new species assigned to the same Category. For this work, the categories and characteristics were used as in Ghoshroy and Caira (2001) and Fyler and Caira (2006) but the character values are as given in the original descriptions or as supplemented by the most recent taxonomic publications. In the Category designation, the type species is identified by number for this classification; the symbol "-" was used for the additional reports of species with additional hosts and/or localities.

For specimens deposited in a formal collection, acronyms are as follows:

AMS

CH-MHNJP Colecciones Helmintológicas del Museo de Historia Natural "Javier Prado” y del Instituto de Medicina Tropical “Daniel. A. Carrión”, Universidad Mayor de San Marcos, Perú;

CHE

CHIOC

CNHE
Colección de Helmintos, Centro de Investigaciones Biológicas, Universidad Autónoma del Estado de Hidalgo, Pachuca, México;

Coleção Helmintológica do Instituto Oswaldo Cruz, Rio de Janeiro, Brazil;

Colección Nacional de Helmintos del Instituto de Biología, Universidad Nacional Autónoma de México, México; 
DMNZ Dominion Musem (=National Museum), New Zealand;

DZAUW Department of Zoology, Andhra University, Waltair, India;

DZCJ

HWML

IPCAS

IPMB

LRP

MACN-Pa

MEPN

MHNLS

MHNP

MLP

MNHG

MNHN

MNHNC

MPM

MZUM (P)

MZUSP

NHMUK

NMNS

PRLXU

QM

SAM AHC

SBC

SPUK

SYSU

UAA

USNPC

ZCUOK

ZIMC

ZMB

ZUTC
Department of Zoology, Bipin Bihari, P. G. College, Jhansi, India;

University of Nebraska State Museum, Harold W. Manter Laboratory, Division of Parasitology, Lincoln, Nebraska, United States;

Institute of Parasitology, Academy of Sciences of the Czech Republic, České Budějovice, Czech Republic;

Institut Penyelidikan Marin Borneo (Borneo Marine Research Institute), Universiti Malaysia Sabah, Kota Kinabalu, Sabah, Malaysia;

Lawrence R. Penner Parasitology Collection, Helminthological Collection, University of Connecticut, Storrs, Connecticut, United States;

Museo Argentino de Ciencias Naturales, Colección Parasitológica, Buenos Aires, Argentina;

Museum of the Escuela Politecnica Nacional, Quito, Ecuador;

Museo de Historia Natural La Salle, Caracas, Venezuela;

Museo de Historia Natural, Lima, Peru;

Museo de Ciencias Naturales de La Plata, Departamento de Zoología Invertebrados (Parasitología), Argentina;

Museum of Natural History, Geneva, Switzerland;

Muséum National d'Histoire Naturelle, Paris;

Museo Nacional de Historia Natural de Chile;

Meguro Parasitology Museum, Tokyo, Japan;

Muzium Zoologi, Universiti Malaya, Kuala Lumpur, Malaysia;

Museu de Zoologia da Universidade de São Paulo, Brazil;

The Natural History Museum, London;

National Museum of Natural Science, Taichung, Taiwan;

Parasitology Research Laboratory, Xiamen University, China;

Queensland Museum, Brisbane, Queensland, Australia;

South Australian Museum, Adelaide, Australia;

Sarawak Biodiversity Center, Kuching, Sarawak, Malaysia;

School of Parasitology, Department of Zoology, University of Karachi, Pakistan;

School of Life Sciences, Sun Yat-sen University;

Department of Zoology, University of Allahabad, Allahabad, India;

United States National Parasite Collection, Beltsville, Maryland, United States;

Zoological Collection, University of Kurdistan, Sanandaj, Iran;

Collection of the Zoological Survey of India, Indian Museum, Calcutta and the Collection of the Department of Zoology, the University of Allahabad, India;

Natural History Museum Berlin, Germany;

Collection of the Zoological Museum, University of Tehran, Tehran, Iran. 


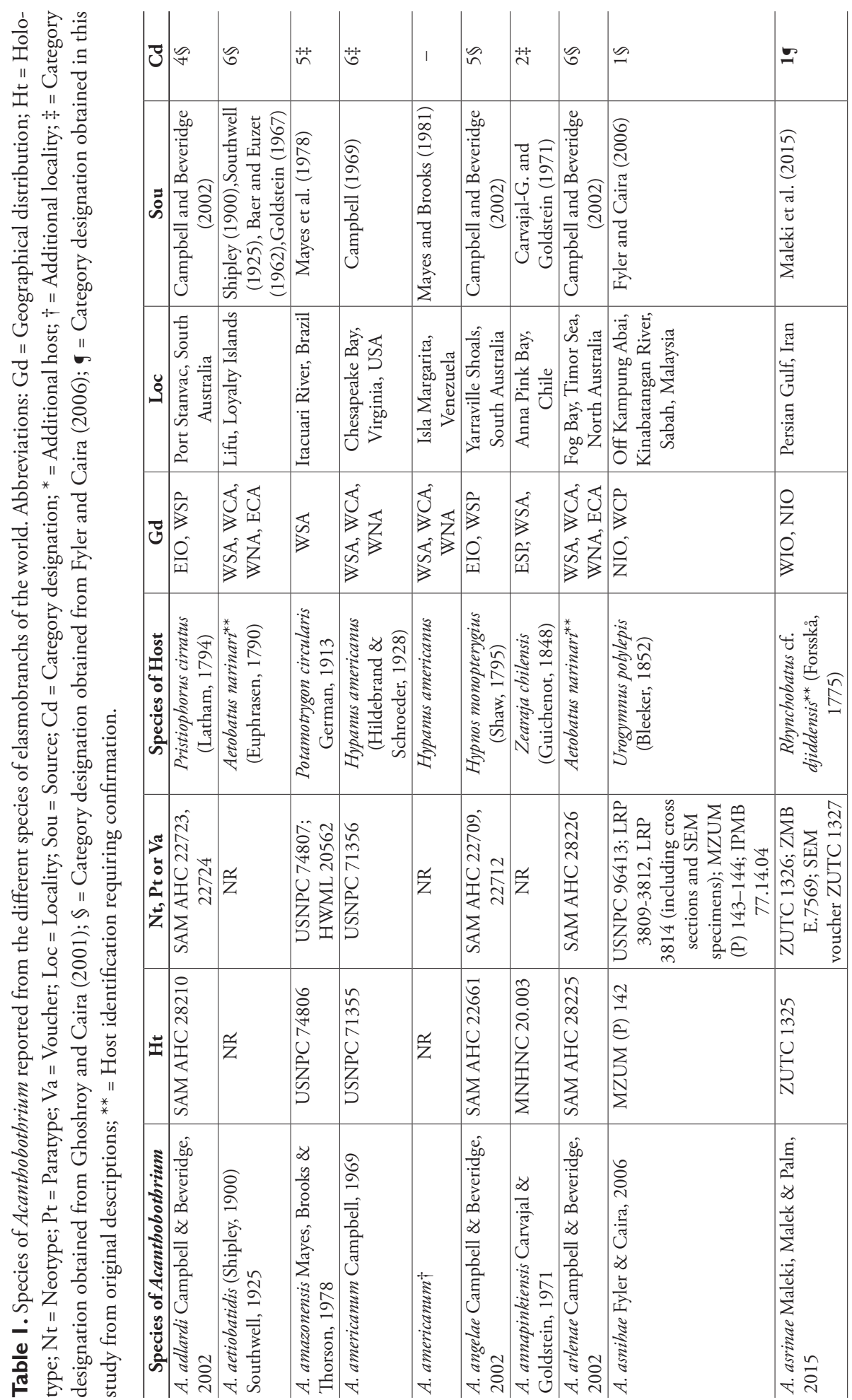




\begin{tabular}{|c|c|c|c|c|c|c|c|c|c|c|c|}
\hline J & $\ddot{+}$ & $\ddot{m}$ & 1 & 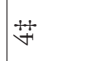 & 1 & $\mathscr{a}$ & $\frac{++}{\mathrm{d}}$ & 1 & 1 & 1 & $\vec{N}$ \\
\hline है & 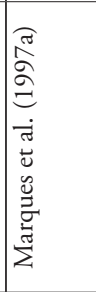 & 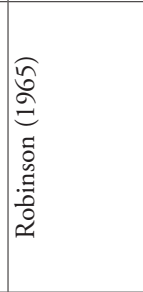 & 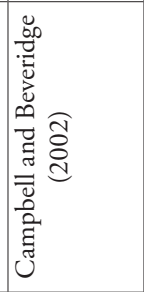 & 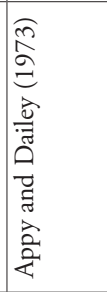 & 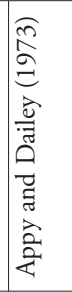 & 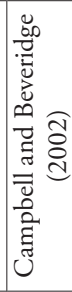 & 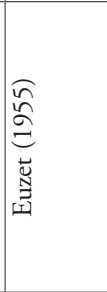 & 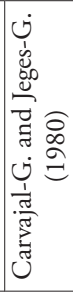 & 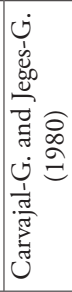 & 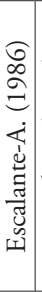 & 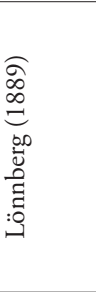 \\
\hline : & 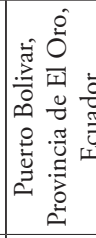 & 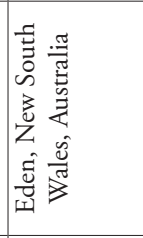 & 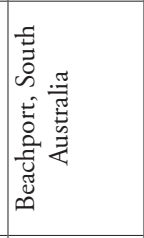 & 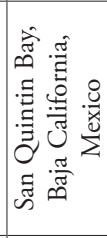 & 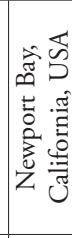 & 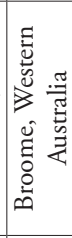 & 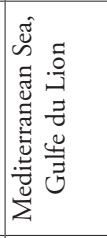 & 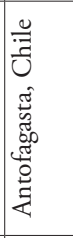 & 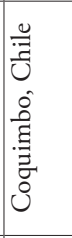 & 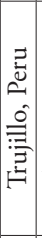 & 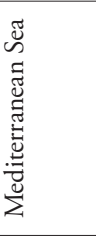 \\
\hline J & $\begin{array}{l}\hat{\tilde{n}} \\
\text { in } \\
\hat{\tilde{u}} \\
\text { In }\end{array}$ & 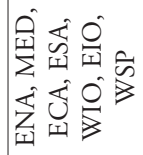 & 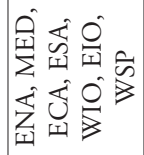 & $\begin{array}{l}\tilde{n} \\
\text { iు } \\
\hat{\tilde{y}} \\
\text { II }\end{array}$ & 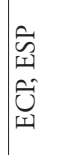 & $\begin{array}{l}0 \\
z \\
0 \\
0 \\
3\end{array}$ & 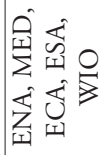 & 荏 & 芯 & 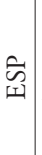 & 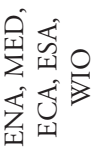 \\
\hline 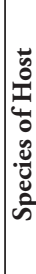 & 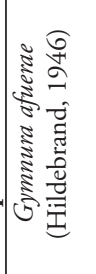 & 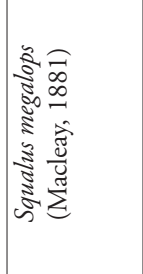 & 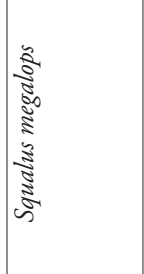 & 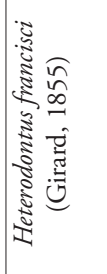 & 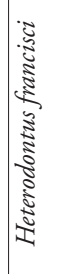 & 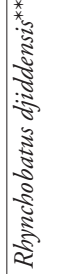 & 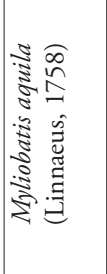 & 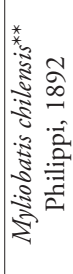 & 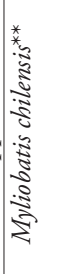 & 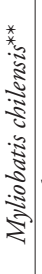 & 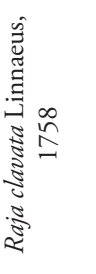 \\
\hline 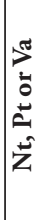 & 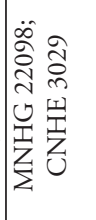 & $\sum_{<}^{\infty}$ & 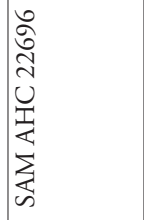 & 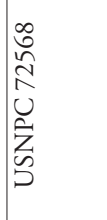 & 号 & 号 & 光 & 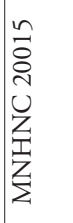 & 孚 & 孚 & 号 \\
\hline 코 & 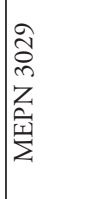 & $\sum_{<}^{\infty}$ & $\frac{\widetilde{z}}{z}$ & 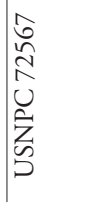 & 亗 & 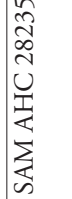 & $\frac{a}{z}$ & $\underline{z}$ & 觉 & 孚 & 岂 \\
\hline 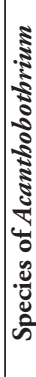 & 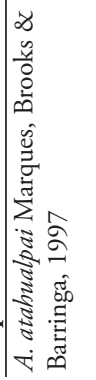 & 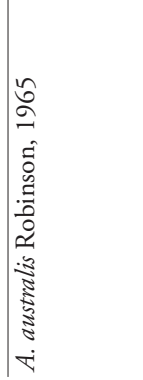 & 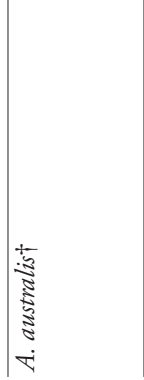 & 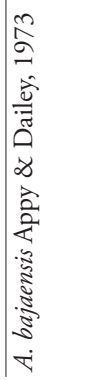 & 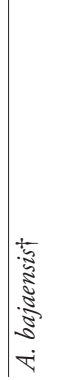 & 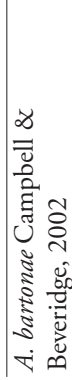 & 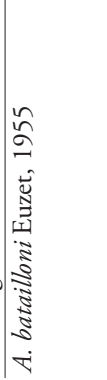 & 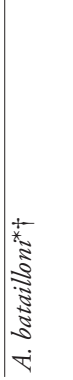 & 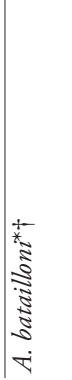 & 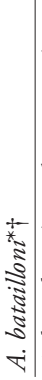 & 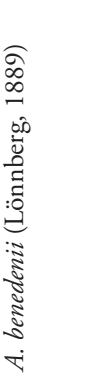 \\
\hline
\end{tabular}




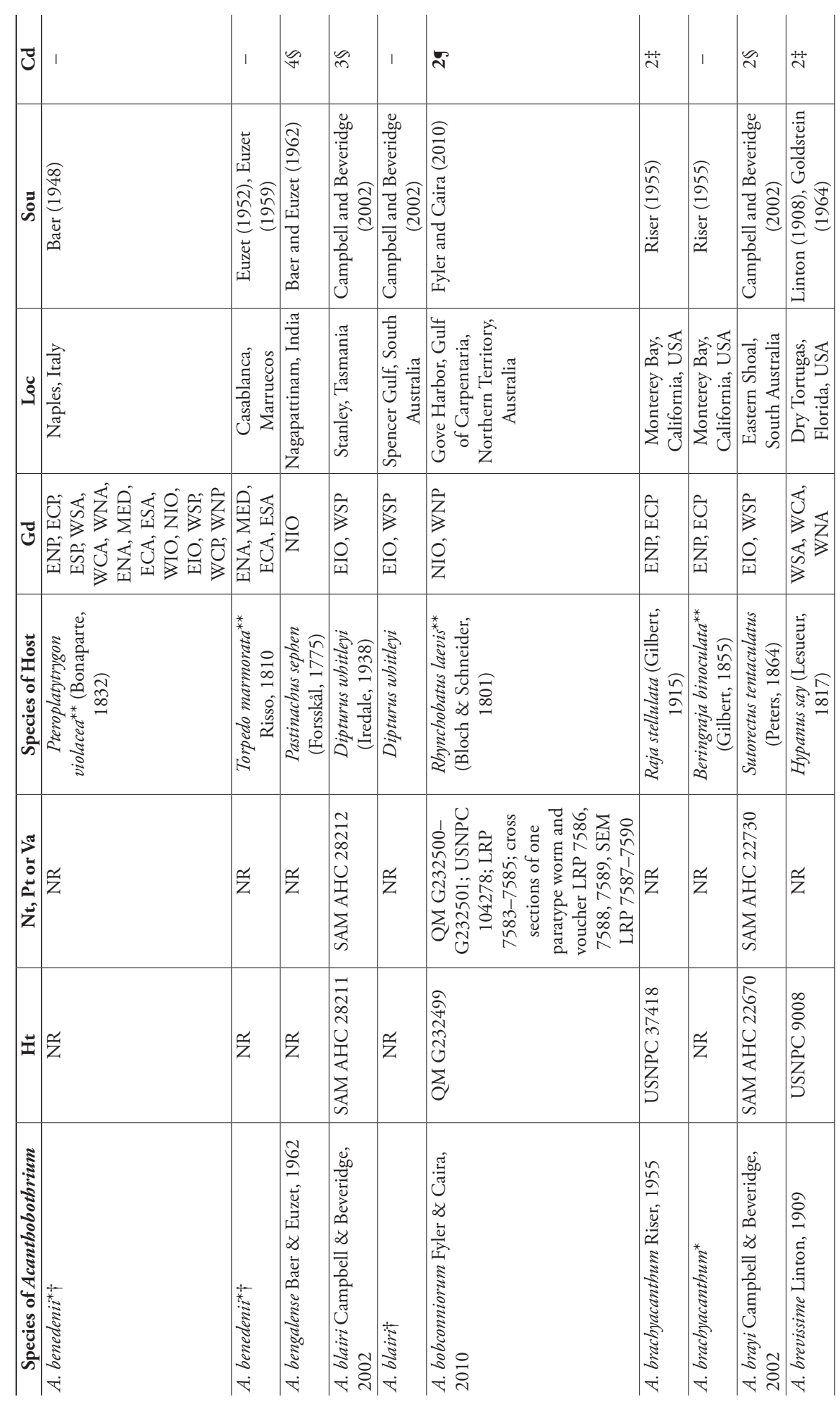




\begin{tabular}{|c|c|c|c|c|c|c|c|c|c|c|c|c|}
\hline$\vec{\jmath}$ & 1 & 1 & 1 & 1 & 茫 & 1 & 1 & $\bar{m}$ & 艺 & 1 & $\underset{\sigma}{\mathscr{q}}$ & $\Xi$ \\
\hline ڤ̈ & 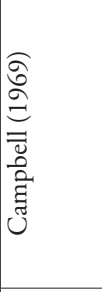 & 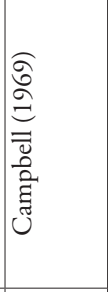 & 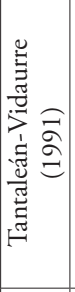 & 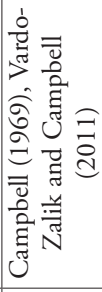 & 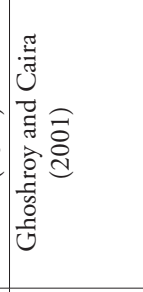 & 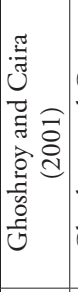 & 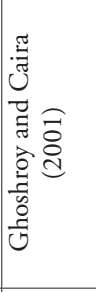 & 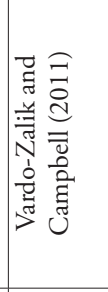 & 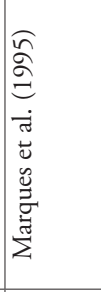 & 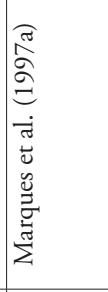 & 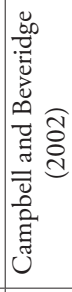 & 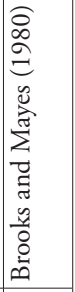 \\
\hline $\mid \begin{array}{l}0 \\
0 \\
\ddots \\
\ddots\end{array}$ & 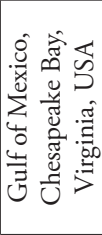 & 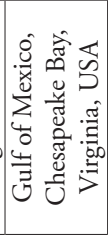 & 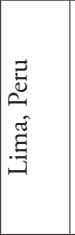 & 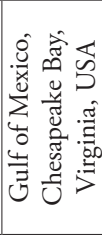 & 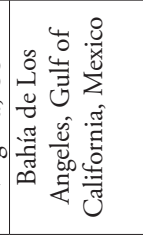 & 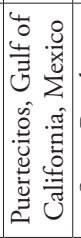 & 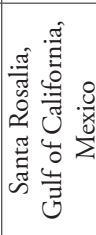 & 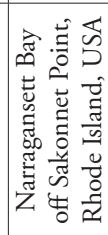 & 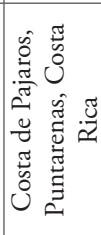 & 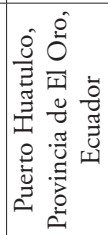 & 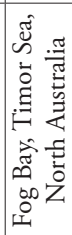 & 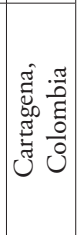 \\
\hline$\vec{J}$ & 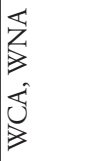 & 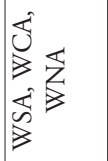 & 荃 & 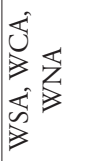 & Uિ & Uี & 诘 & 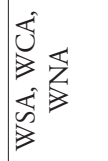 & $\begin{array}{l}\hat{n} \\
\text { II } \\
\hat{\tilde{y}} \\
\text { i= }\end{array}$ & 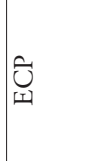 & 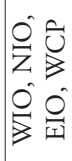 & 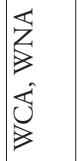 \\
\hline 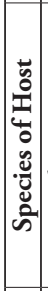 & 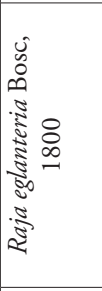 & 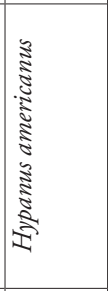 & 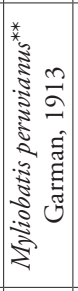 & 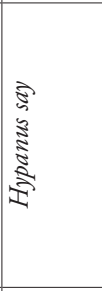 & 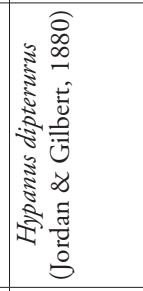 & 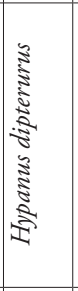 & 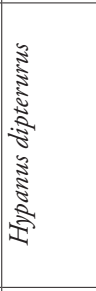 & 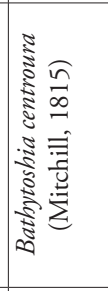 & 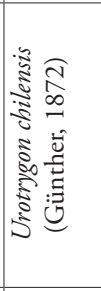 & 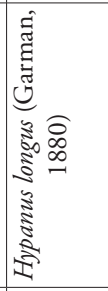 & 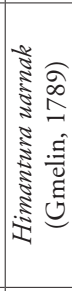 & 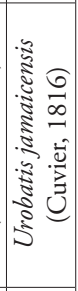 \\
\hline 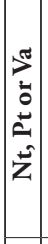 & 号 & 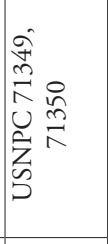 & 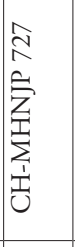 & 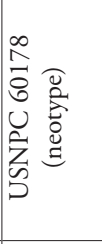 & 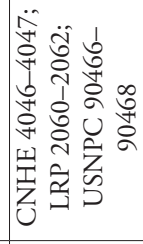 & 光 & 岁 & 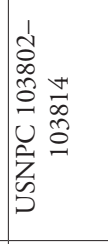 & 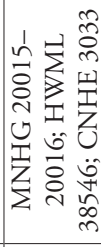 & 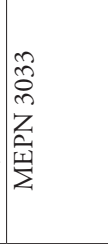 & 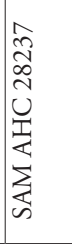 & $\underline{z}$ \\
\hline $\mathbf{\pm}$ & 孚 & 光 & 孚 & 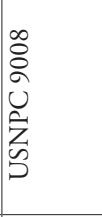 & 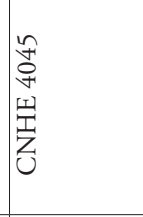 & 占 & 孚 & $\begin{array}{l}\tilde{D} \\
\infty \\
\infty \\
0 \\
0 \\
\tilde{z} \\
\tilde{D} \\
\rho\end{array}$ & 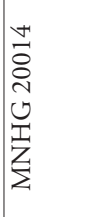 & $\tilde{z}$ & 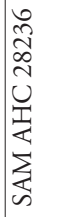 & $\begin{array}{l}\hat{n} \\
n \\
u \\
u \\
\tilde{z} \\
\tilde{n}\end{array}$ \\
\hline 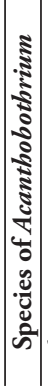 & 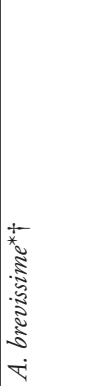 & 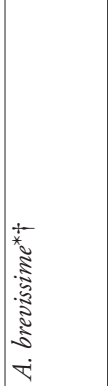 & 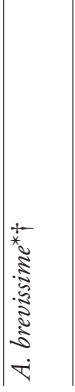 & 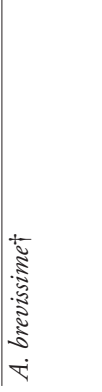 & 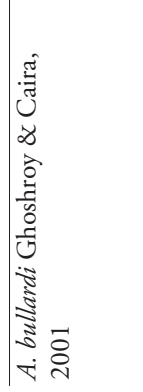 & 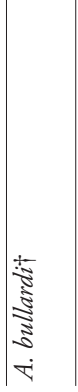 & $\mid \begin{array}{l}3 \\
8 \\
-1\end{array}$ & 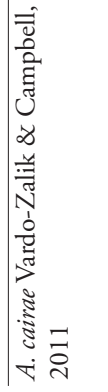 & 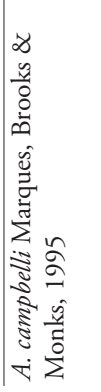 & 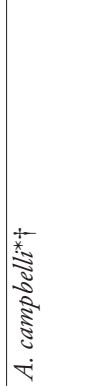 & 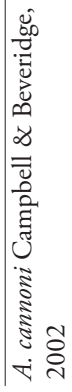 & 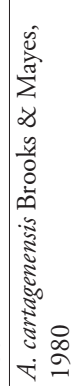 \\
\hline
\end{tabular}




\begin{tabular}{|c|c|c|c|c|c|c|c|c|c|c|}
\hline & & & & 8 & । & $=$ & m & 芦 & 苛 & $\cong$ \\
\hline 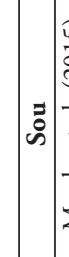 & 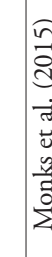 & & & 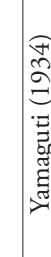 & 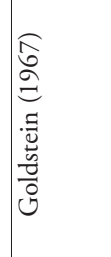 & 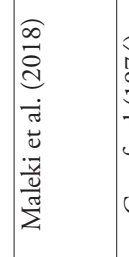 & 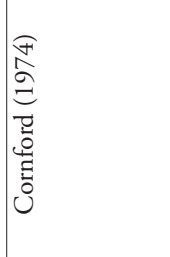 & 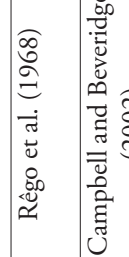 & & 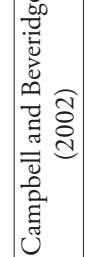 \\
\hline & & & & 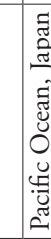 & 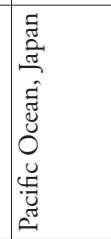 & 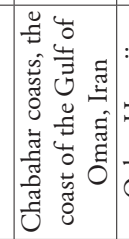 & 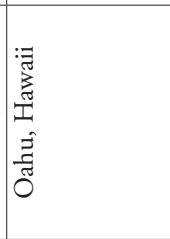 & 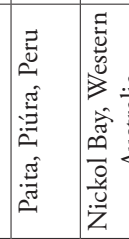 & & 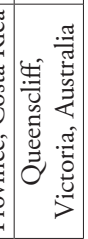 \\
\hline $\mid$ & $\bar{z}$ & & & $\begin{array}{l}0 \\
\hat{B} \\
\hat{z}\end{array}$ & & $\frac{\rho}{z}$ & 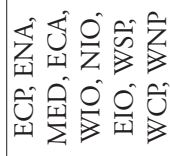 & $\frac{0}{9}$ & 謟 & 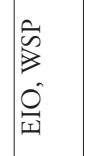 \\
\hline ڤั้ & 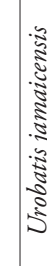 & & & & 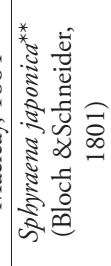 & 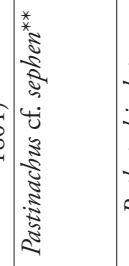 & 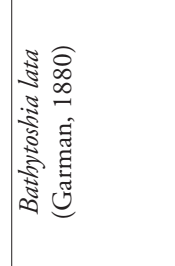 & 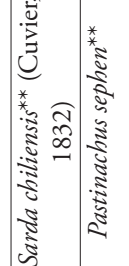 & 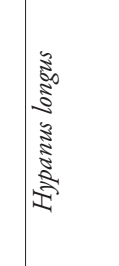 & 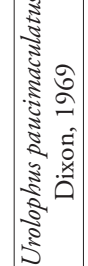 \\
\hline & & & & & $\tilde{z}$ & 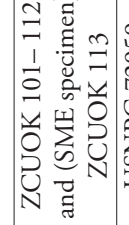 & & 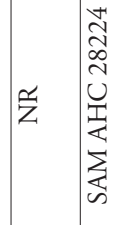 & 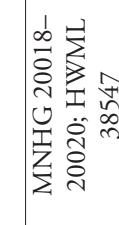 & 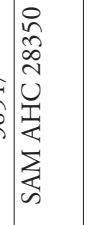 \\
\hline \pm & ż & & & ź & $\tilde{z}$ & $\begin{array}{l}8 \\
0 \\
0 \\
0 \\
0 \\
0 \\
N\end{array}$ & 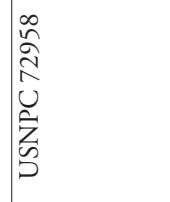 & 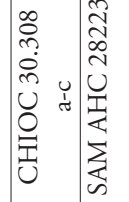 & 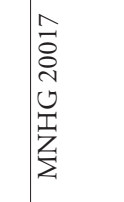 & 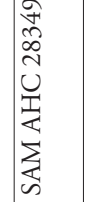 \\
\hline $\mid$ & & & & t & प्र & 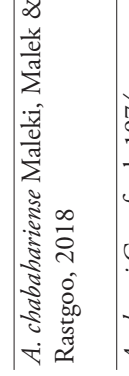 & 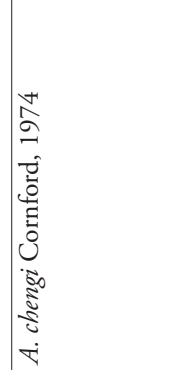 & 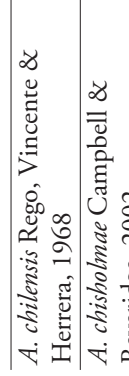 & 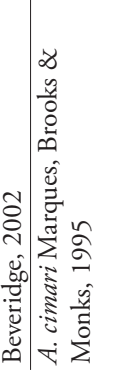 & 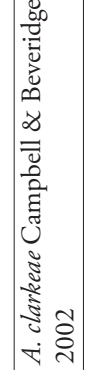 \\
\hline
\end{tabular}




\begin{tabular}{|c|c|c|c|c|c|c|c|c|c|c|c|c|c|c|c|c|c|}
\hline 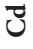 & I & 1 & $\ddot{m}$ & $\ddot{\alpha}$ & $\approx$ & $\dot{\sim}$ & 1 & $\mathscr{\sigma}$ & 1 & 1 & 1 & 1 & 1 & 1 & 1 & 1 & 1 \\
\hline है & 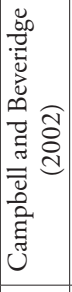 & 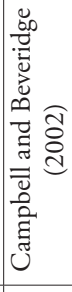 & 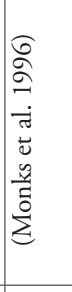 & 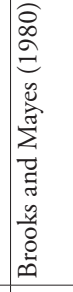 & 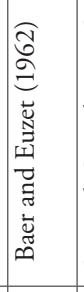 & 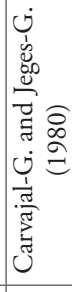 & 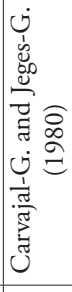 & 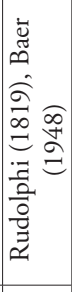 & 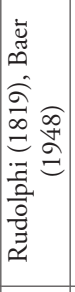 & 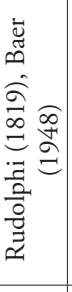 & 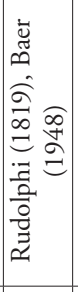 & 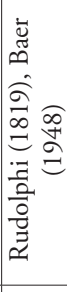 & 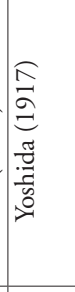 & 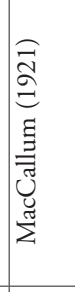 & 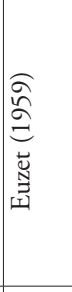 & 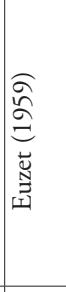 & 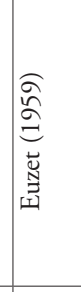 \\
\hline 8 & 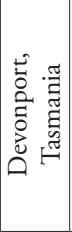 & 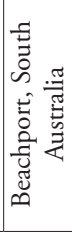 & 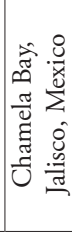 & 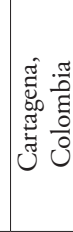 & 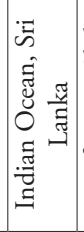 & 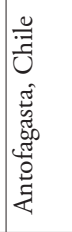 & 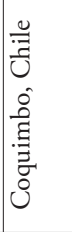 & 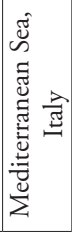 & 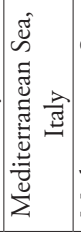 & 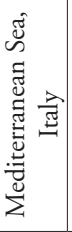 & 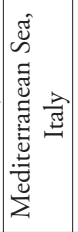 & 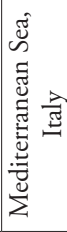 & 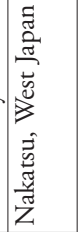 & 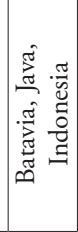 & 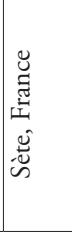 & 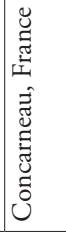 & 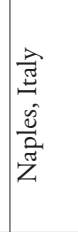 \\
\hline & $\overbrace{-1}^{0}$ & $\stackrel{0}{\stackrel{0}{I I}}$ & 仑્ & 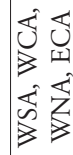 & $\tilde{s}$ & $\begin{array}{l}\hat{n} \\
\text { tI }\end{array}$ & $\mid \begin{array}{l}\tilde{\tilde{n}} \\
\text { II }\end{array}$ & 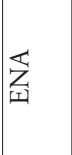 & 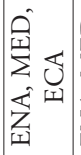 & 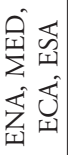 & 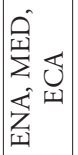 & 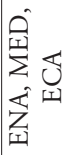 & $\tilde{z}$ & 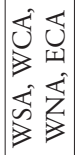 & 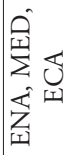 & 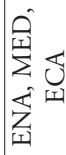 & 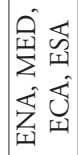 \\
\hline & 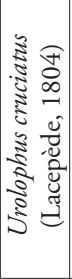 & 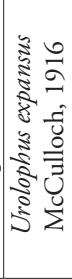 & 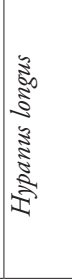 & 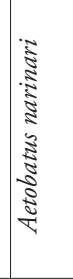 & 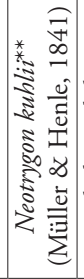 & 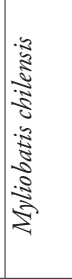 & 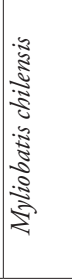 & 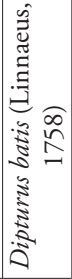 & 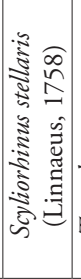 & 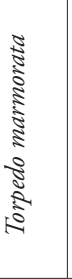 & 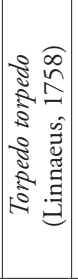 & 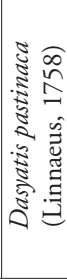 & 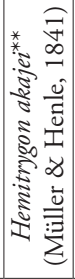 & 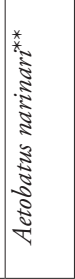 & 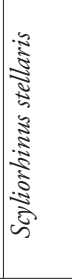 & 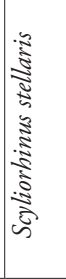 & 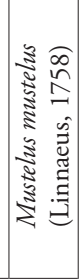 \\
\hline
\end{tabular}

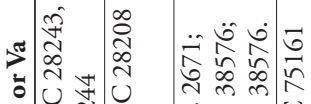
帘

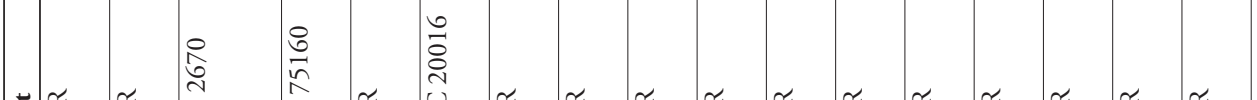

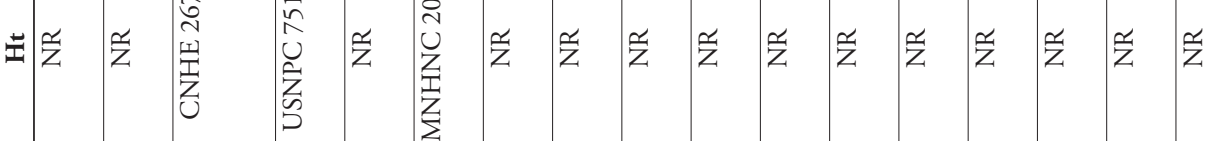

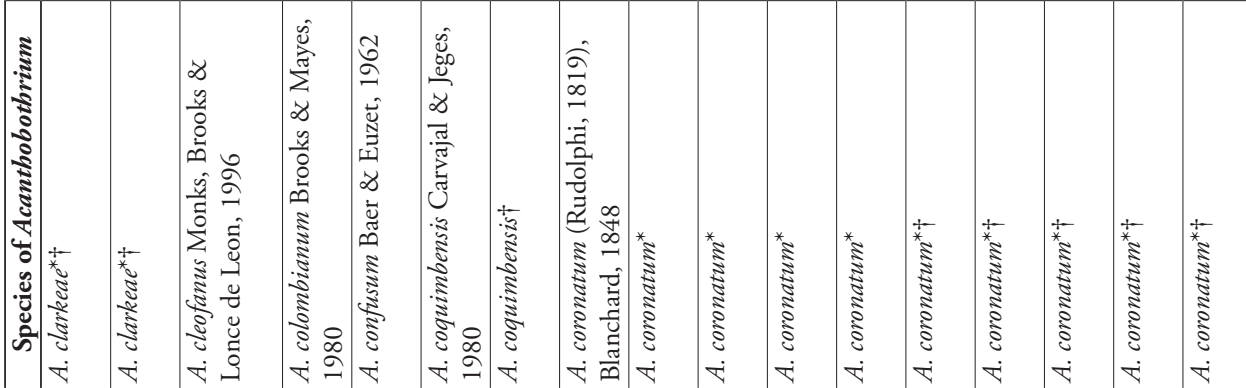




\begin{tabular}{|c|c|c|c|c|c|c|c|c|c|c|c|c|c|}
\hline $\bar{U}$ & I & 1 & 1 & $\ddot{\sim}$ & 1 & $\ddot{m}$ & $\mathscr{\sigma}$ & $\ddot{\sim}$ & 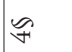 & 1 & 1 & $\approx$ & $=$ \\
\hline & 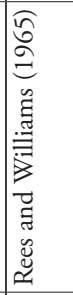 & 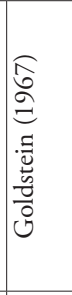 & 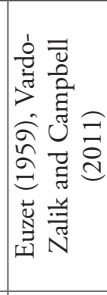 & 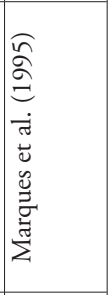 & 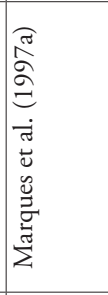 & 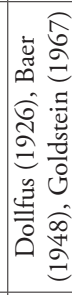 & 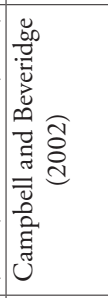 & 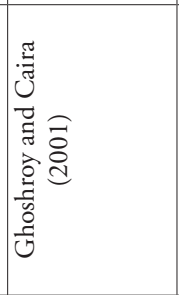 & 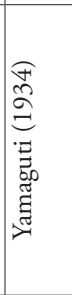 & 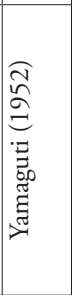 & 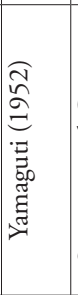 & 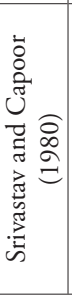 & 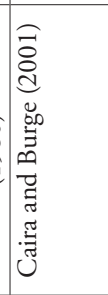 \\
\hline & 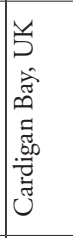 & 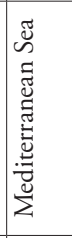 & 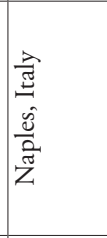 & 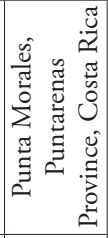 & 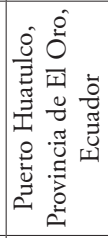 & 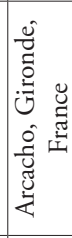 & 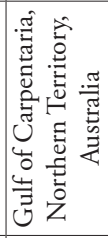 & 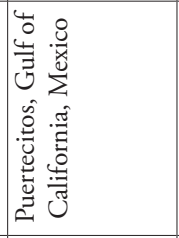 & 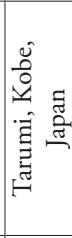 & 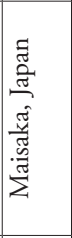 & 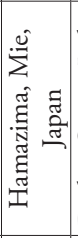 & 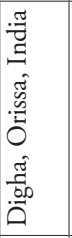 & 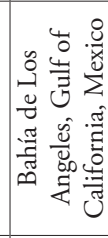 \\
\hline$\vec{J}$ & 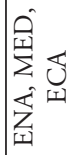 & $\stackrel{0}{\stackrel{1}{\Sigma}}$ & 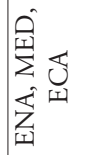 & Uি & 式 & 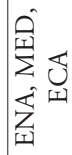 & 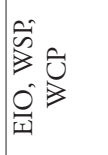 & 式 & $\underline{z}$ & $\vec{z}$ & n. & $\begin{array}{l}\tilde{U} \\
\dot{y} \\
0 \\
\bar{Z}\end{array}$ & 式 \\
\hline 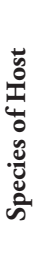 & 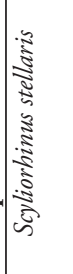 & 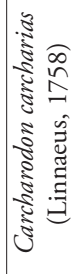 & 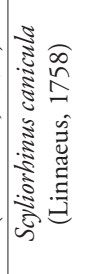 & 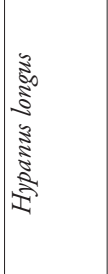 & 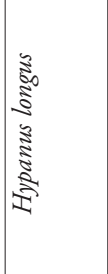 & 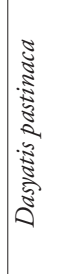 & 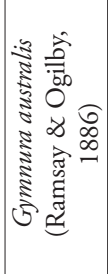 & 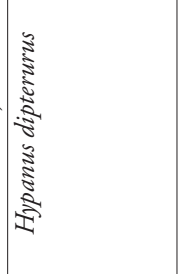 & 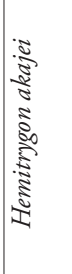 & 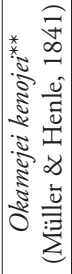 & 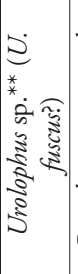 & 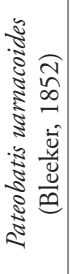 & 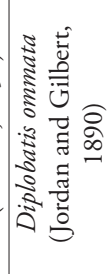 \\
\hline 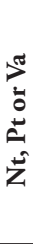 & 妾 & 孚 & 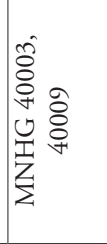 & 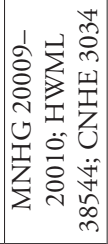 & 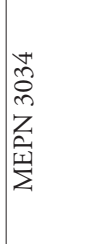 & 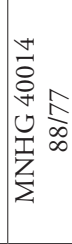 & 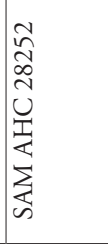 & 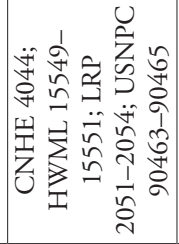 & 㟔 & 孚 & 孚 & 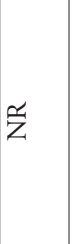 & 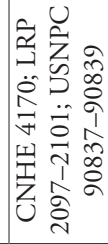 \\
\hline 击 & 孚 & 觉 & $\tilde{z}$ & 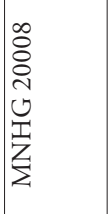 & $\tilde{z}$ & 觉 & 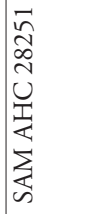 & 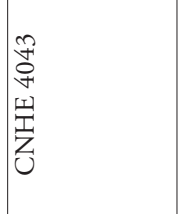 & 觉 & 孚 & 点 & $\frac{5}{5}$ & 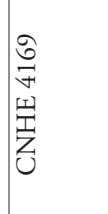 \\
\hline 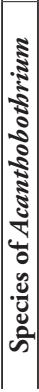 & 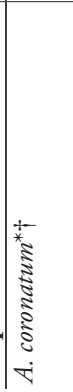 & 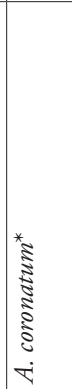 & 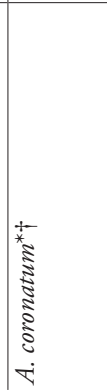 & 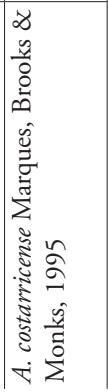 & 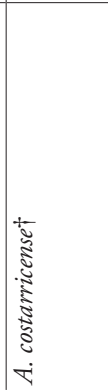 & 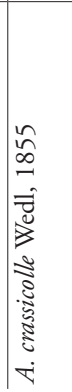 & 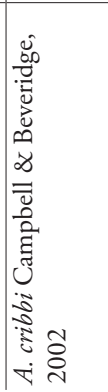 & 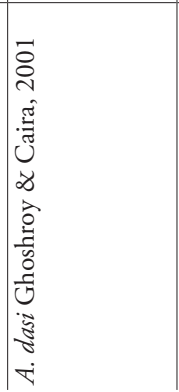 & 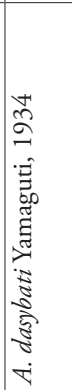 & 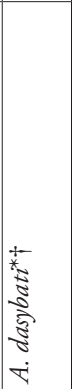 & 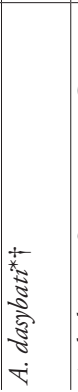 & 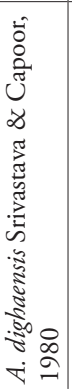 & 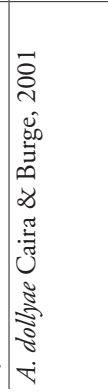 \\
\hline
\end{tabular}




\begin{tabular}{|c|c|c|c|c|c|c|c|c|c|c|c|c|c|}
\hline 3 & I & 1 & $\mathscr{\sim}$ & 1 & 1 & 1 & 尽 & $\approx$ & 1 & 1 & $\widetilde{\sim}$ & 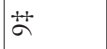 & $t$ \\
\hline 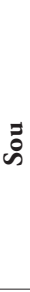 & 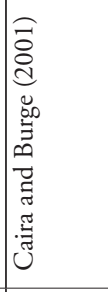 & 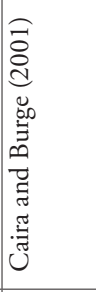 & 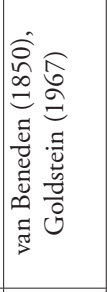 & 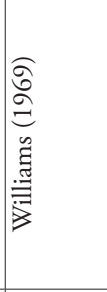 & 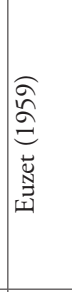 & 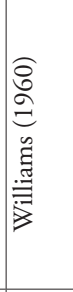 & 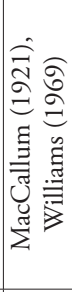 & 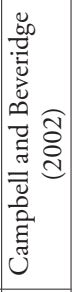 & 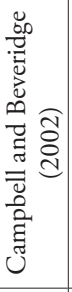 & 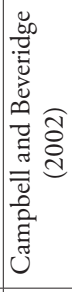 & 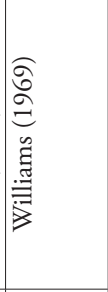 & 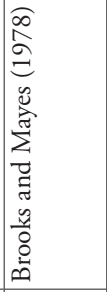 & \\
\hline ְּ & 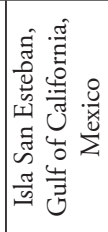 & 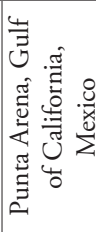 & 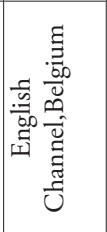 & 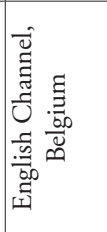 & 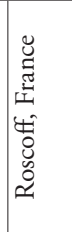 & 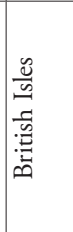 & 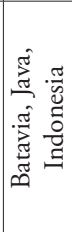 & 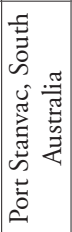 & 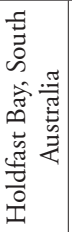 & 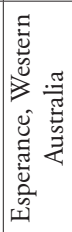 & 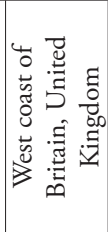 & 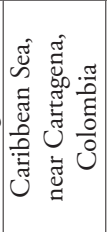 & \\
\hline Jु & 式 & 對 & 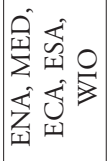 & 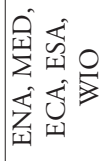 & 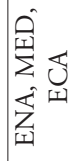 & 裁 & 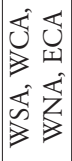 & $\begin{array}{l}\vec{n} \\
0 \\
0 \\
0\end{array}$ & $\begin{array}{l}\hat{w} \\
\vdots \\
0 \\
0 \\
1\end{array}$ & 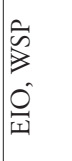 & 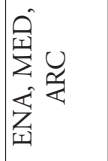 & 芯 & \\
\hline 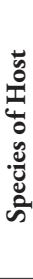 & 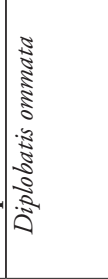 & 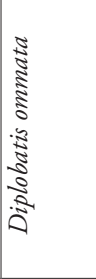 & 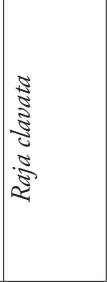 & 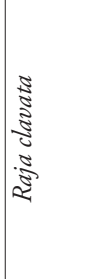 & 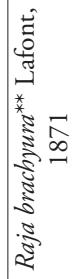 & 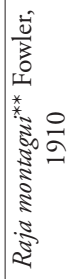 & 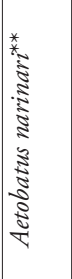 & 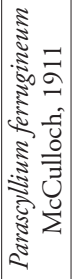 & 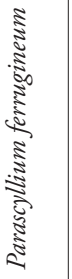 & 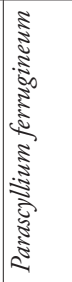 & 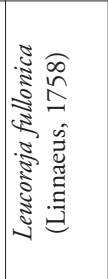 & 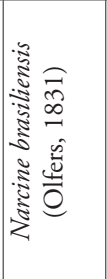 & \\
\hline $\overrightarrow{0}$ & 妾 & 孚 & 占 & $\tilde{z}$ & 孚 & 光 & 点 & 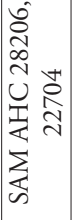 & 㟔 & 孚 & 孚 & 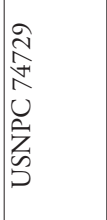 & \\
\hline \pm & 觉 & 孚 & 点 & 点 & 光 & 孚 & 孚 & 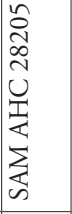 & 岂 & 号 & $\tilde{z}$ & 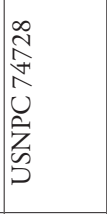 & \\
\hline . & 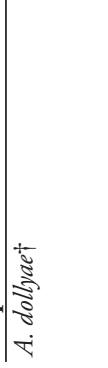 & $\begin{array}{l}+\frac{1}{3} \\
\text { בे } \\
8 \\
\dot{8}\end{array}$ & 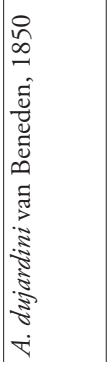 & 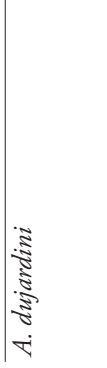 & 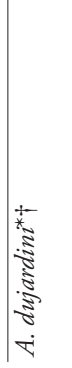 & 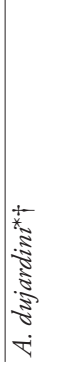 & 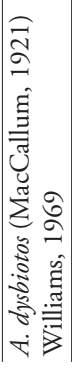 & 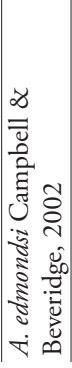 & $\begin{array}{l}\text { है } \\
\text { सं }\end{array}$ & 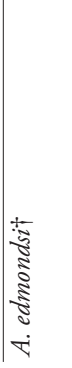 & 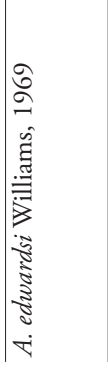 & 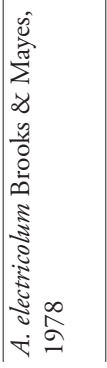 & \\
\hline
\end{tabular}




\begin{tabular}{|c|c|c|c|c|c|c|c|c|c|c|}
\hline $\bar{U}$ & $\infty$ & $\underset{\infty}{\stackrel{\infty}{\infty}}$ & 1 & $\underset{\infty}{\stackrel{++}{O}}$ & 1 & 1 & $\#$ & 1 & $=$ & $\frac{++}{\stackrel{+\infty}{\infty}}$ \\
\hline है & 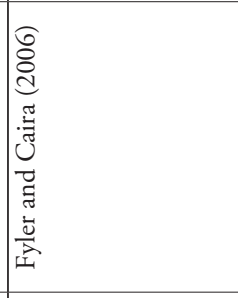 & 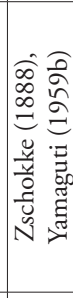 & 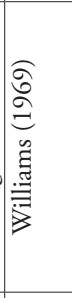 & 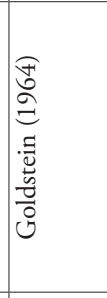 & 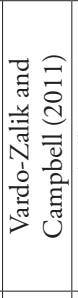 & 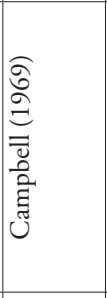 & 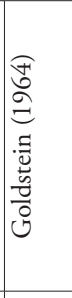 & 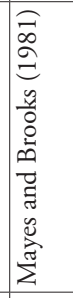 & 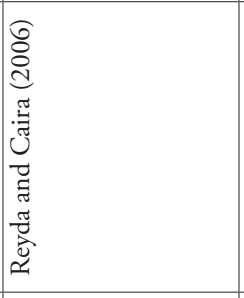 & 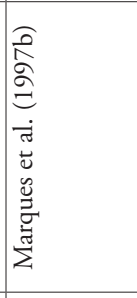 \\
\hline & 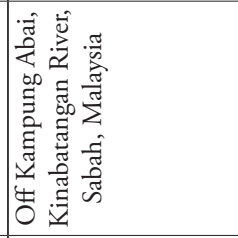 & 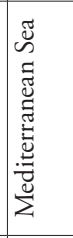 & 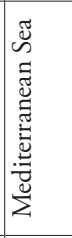 & 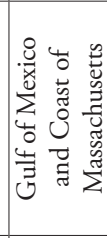 & 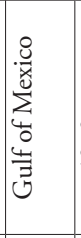 & 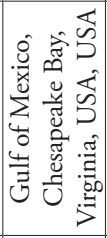 & 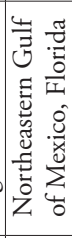 & 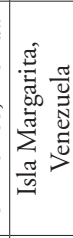 & 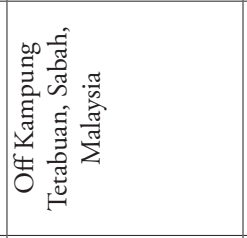 & 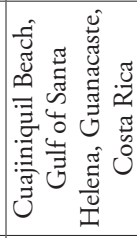 \\
\hline J゙ & $\begin{array}{l}\tilde{U} \\
\vdots \\
0 \\
Z\end{array}$ & 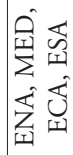 & 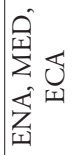 & $\begin{array}{l}\overleftrightarrow{3} \\
3 \\
3 \\
3\end{array}$ & 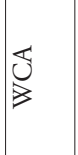 & 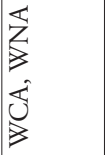 & 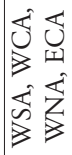 & 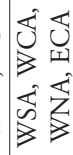 & $\begin{array}{l}\hat{0} \\
\vdots \\
0 \\
\vdots \\
Z\end{array}$ & $\underbrace{}_{\text {II }}$ \\
\hline 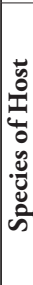 & 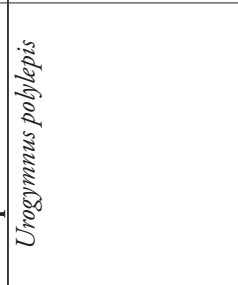 & 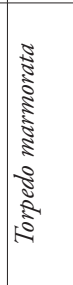 & 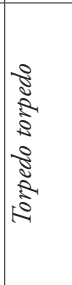 & 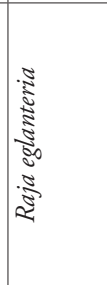 & 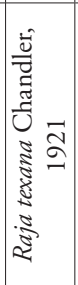 & 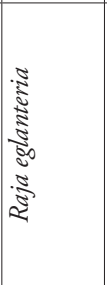 & 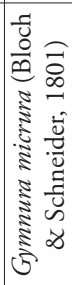 & $\frac{\sqrt{3}}{\sqrt{2}}$ & 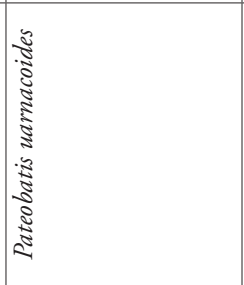 & 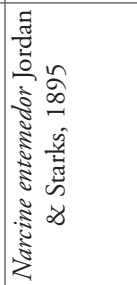 \\
\hline 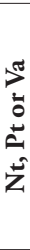 & 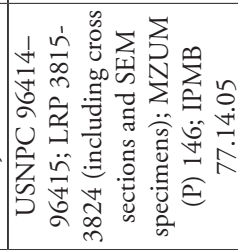 & 艺 & 危 & $\tilde{z}$ & 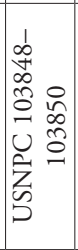 & 觉 & 号 & 㟔 & 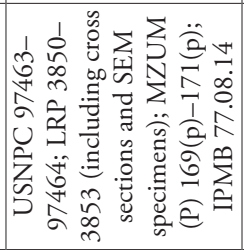 & 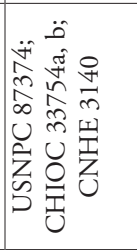 \\
\hline 考 & 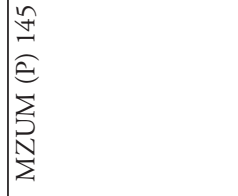 & $\tilde{z}$ & 孚 & 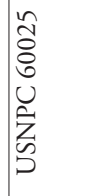 & 觉 & 孚 & 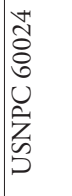 & 艺 & 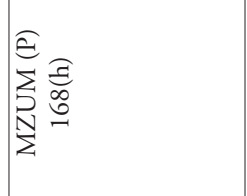 & 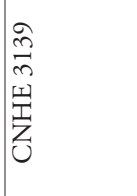 \\
\hline & 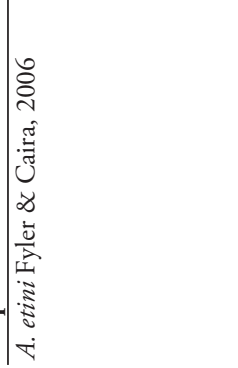 & 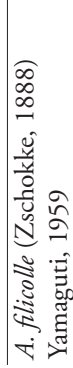 & 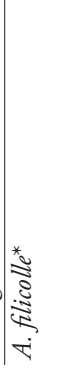 & 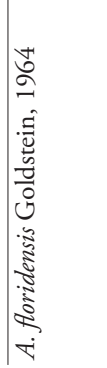 & 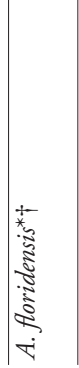 & 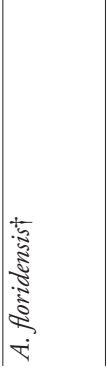 & 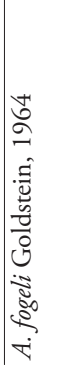 & 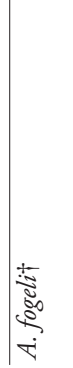 & 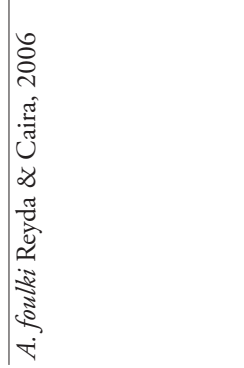 & 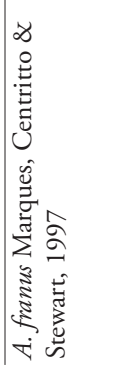 \\
\hline
\end{tabular}




\begin{tabular}{|c|c|c|c|c|c|c|c|c|c|c|c|c|}
\hline$\vec{J}$ & $=$ & $\stackrel{m}{m}$ & $\ddot{n}$ & $\approx$ & $\approx$ & $=$ & 菾 & $\frac{6}{6}$ & $\ddot{m}$ & $\mathscr{q}$ & 1 & $\stackrel{\sim}{\sim}$ \\
\hline $\bar{b}$ & 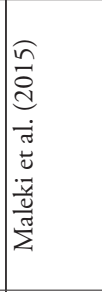 & 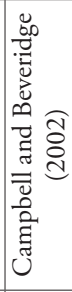 & 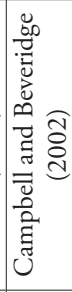 & 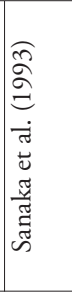 & 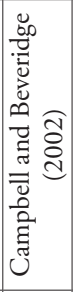 & 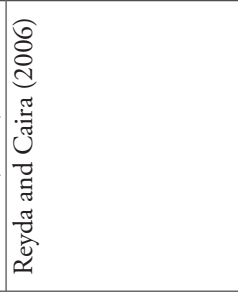 & 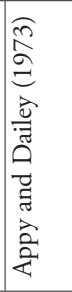 & 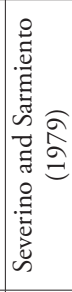 & 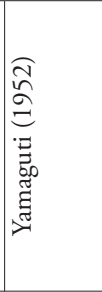 & 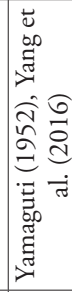 & 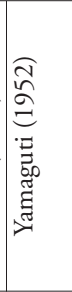 & 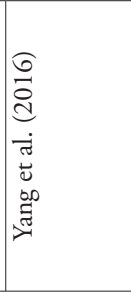 \\
\hline 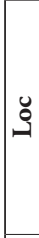 & 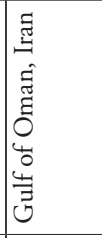 & 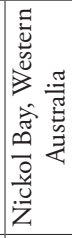 & 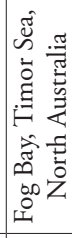 & 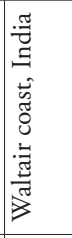 & 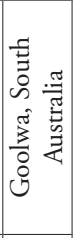 & 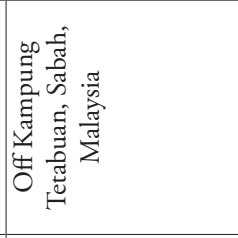 & 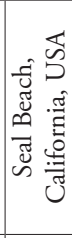 & 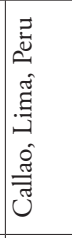 & 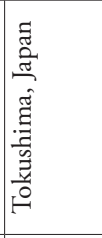 & 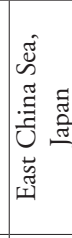 & 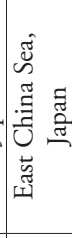 & 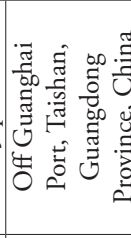 \\
\hline$\vec{J}$ & $\begin{array}{l}0 \\
\vdots z \\
0 \\
0 \\
3\end{array}$ & $\stackrel{0}{Z}$ & $\begin{array}{l}0 \\
Z \\
z \\
0 \\
3 \\
3\end{array}$ & 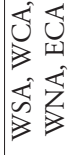 & $\tilde{\tilde{n}}$ & $\begin{array}{l}0 \\
0 \\
0 \\
0 \\
\bar{z}\end{array}$ & 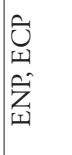 & $\begin{array}{l}\hat{\bar{s}} \\
\bar{y}\end{array}$ & $\hat{z}$ & $\begin{array}{l}\hat{z} \\
\hat{z} \\
\hat{j} \\
\geqslant\end{array}$ & $\hat{z}$ & $\hat{z}$ \\
\hline & 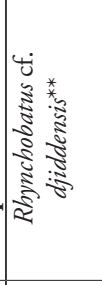 & 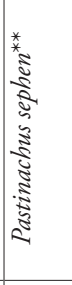 & 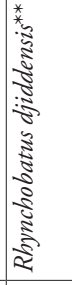 & 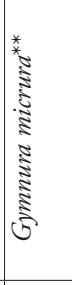 & 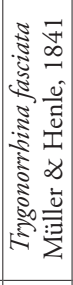 & 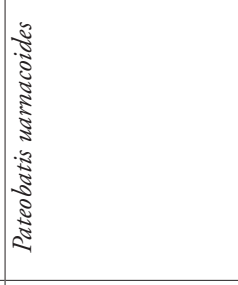 & 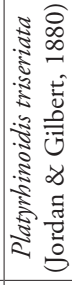 & 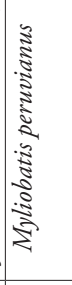 & 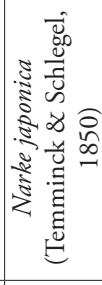 & 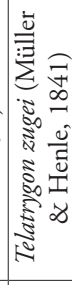 & 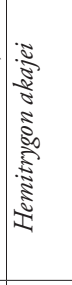 & 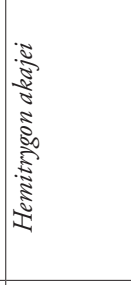 \\
\hline $\begin{array}{l}5 \\
5 \\
5 \\
5 \\
5 \\
5\end{array}$ & 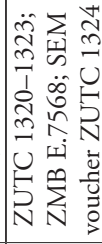 & 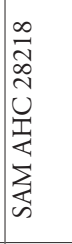 & 光 & 孚 & 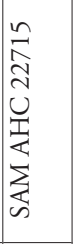 & 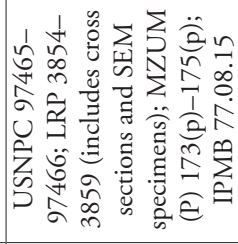 & 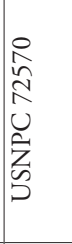 & 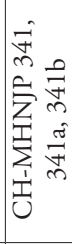 & $\frac{a}{z}$ & 㟔 & $\tilde{z}$ & 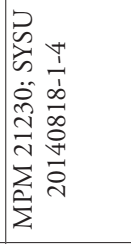 \\
\hline \pm & 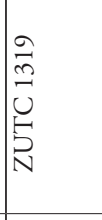 & 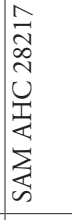 & 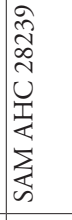 & 点 & 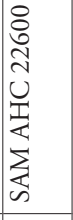 & 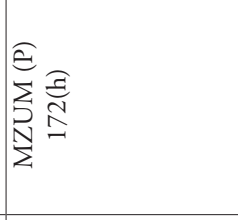 & 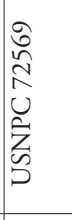 & 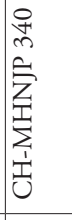 & 岂 & 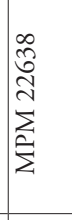 & $\tilde{z}$ & 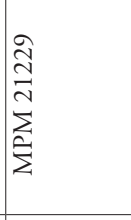 \\
\hline 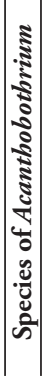 & 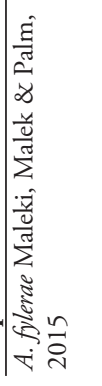 & 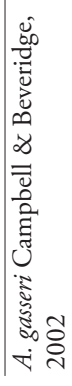 & 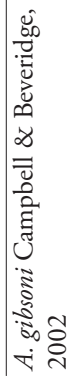 & 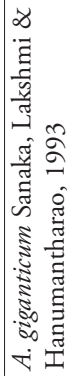 & 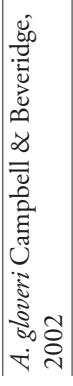 & 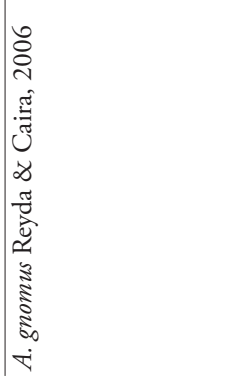 & 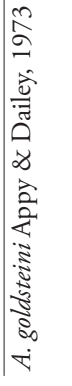 & 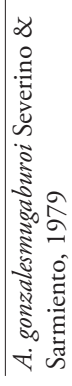 & 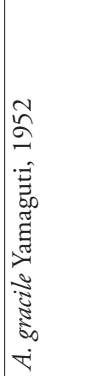 & 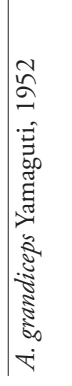 & 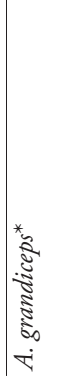 & 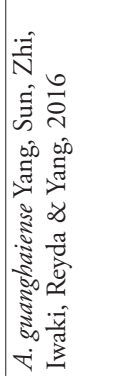 \\
\hline
\end{tabular}




\begin{tabular}{|c|c|c|c|c|c|c|c|c|c|c|c|}
\hline J & $=$ & $\mathscr{\sigma}$ & $\bar{m}$ & $\mathscr{\sigma}$ & 1 & 1 & $\ddot{+}$ & in & 莦 & 1 & $\bar{d}$ \\
\hline$\ddot{b}$ & 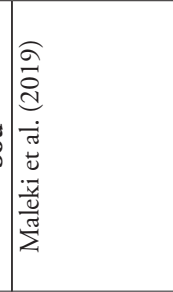 & 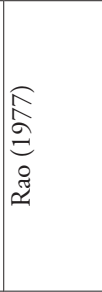 & 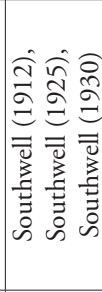 & 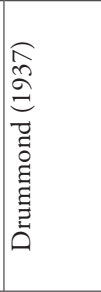 & 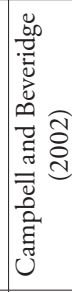 & 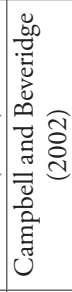 & 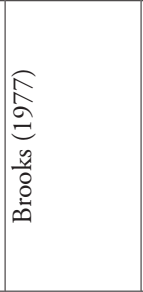 & 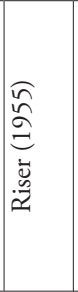 & 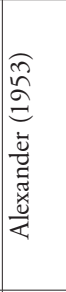 & 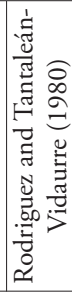 & 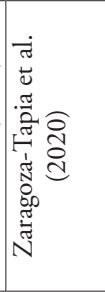 \\
\hline : & 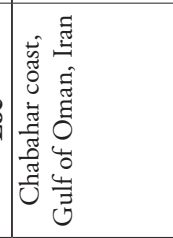 & 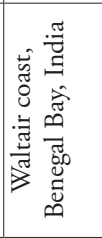 & 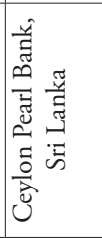 & 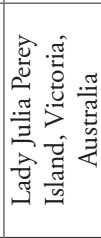 & 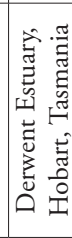 & 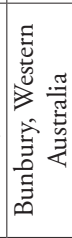 & 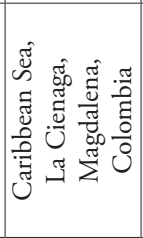 & 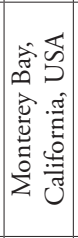 & 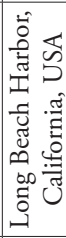 & 离 & 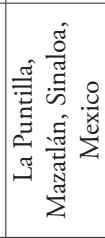 \\
\hline تु & 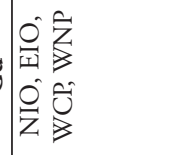 & 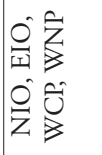 & $\hat{\tilde{z}}$ & $\begin{array}{l}\hat{\tilde{n}} \\
\hat{8} \\
0 \\
0 \\
1.1\end{array}$ & $\begin{array}{l}\hat{5} \\
0 \\
0 \\
0 \\
0\end{array}$ & $\begin{array}{l}\hat{\tilde{n}} \\
\hat{8} \\
0 \\
0\end{array}$ & 出 & $\begin{array}{l}\hat{\tilde{y}} \\
\dot{y} \\
\hat{\bar{z}} \\
\hat{y}\end{array}$ & $\begin{array}{l}\hat{0} \\
\text { 任 } \\
\hat{\bar{Z}} \\
\text { III }\end{array}$ & $\begin{array}{l}\hat{\tilde{n}} \\
\text { II }\end{array}$ & $\hat{\text { ț }}$ \\
\hline 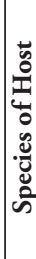 & 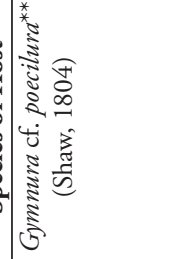 & 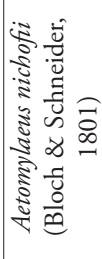 & 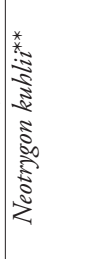 & 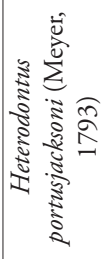 & 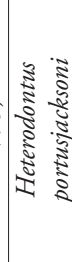 & 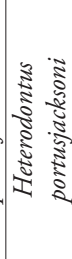 & 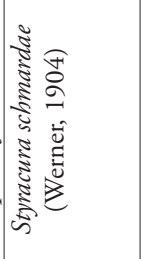 & 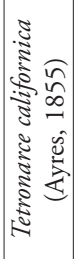 & 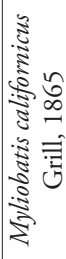 & 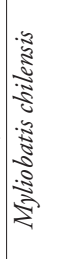 & 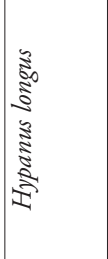 \\
\hline ثَّ & 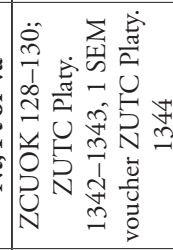 & 艺 & $\tilde{z}$ & 光 & 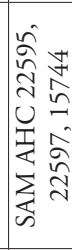 & 光 & 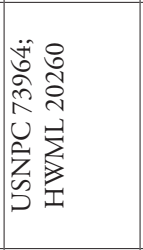 & 学 & 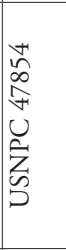 & 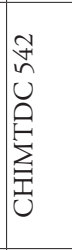 & 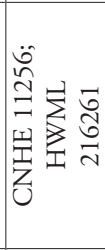 \\
\hline 丰 & 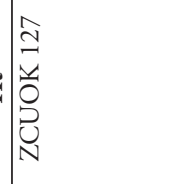 & 孚 & 孚 & 孚 & 觉 & 觉 & 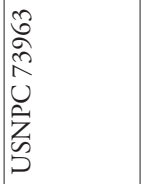 & 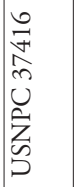 & 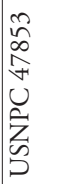 & 觉 & 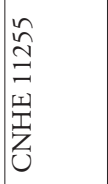 \\
\hline 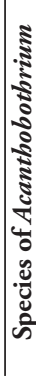 & 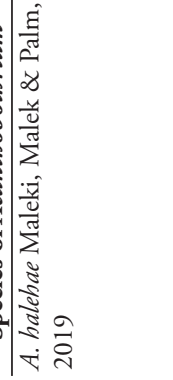 & 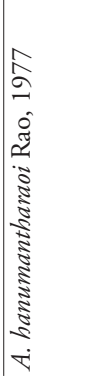 & 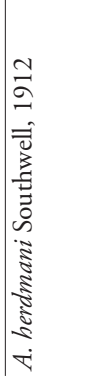 & 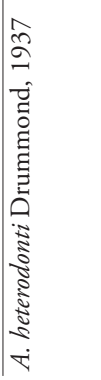 & 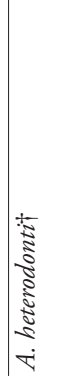 & 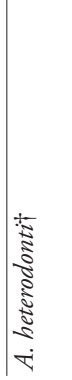 & 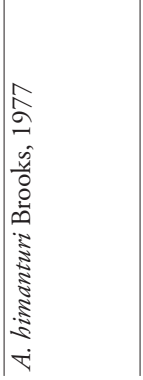 & 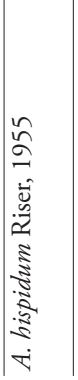 & 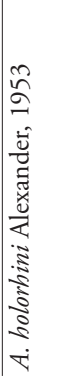 & 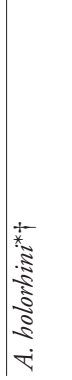 & 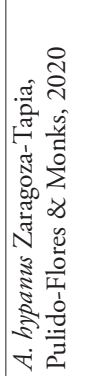 \\
\hline
\end{tabular}




\begin{tabular}{|c|c|c|c|c|c|c|c|c|c|c|c|}
\hline$\vec{u}$ & $\Xi$ & $\ddot{m}$ & \& & 1 & 茫 & s. & $\approx$ & 㷊 & $\Xi$ & $=$ & $=$ \\
\hline $\overrightarrow{\tilde{b}}$ & 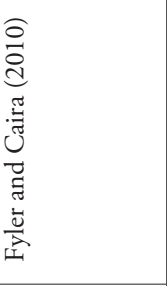 & 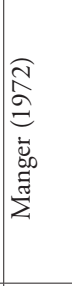 & 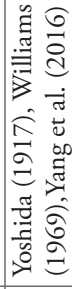 & 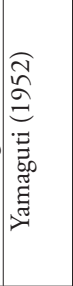 & 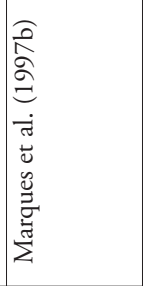 & 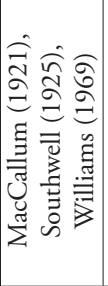 & 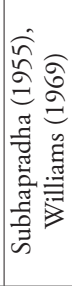 & 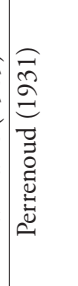 & 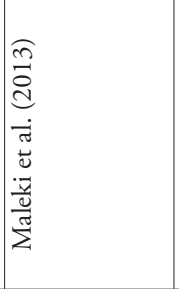 & 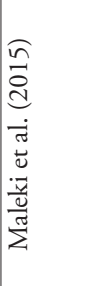 & 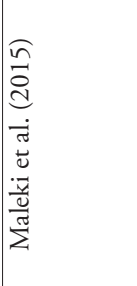 \\
\hline & 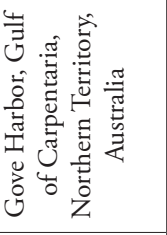 & 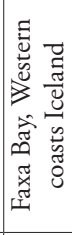 & 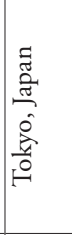 & 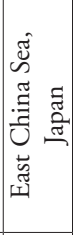 & 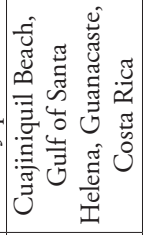 & 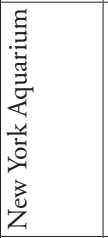 & 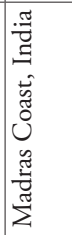 & 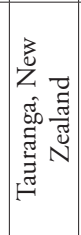 & 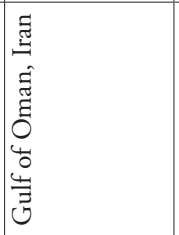 & 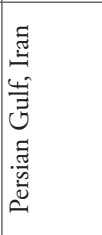 & 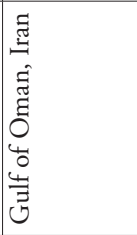 \\
\hline$\vec{J}$ & $\begin{array}{l}\hat{z} \\
\vdots \\
0 \\
\vdots \\
z\end{array}$ & $\overleftrightarrow{Z}$ & $\tilde{z}$ & $\bar{z}$ & 式 & 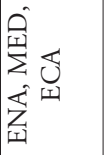 & $\underset{n}{\mathscr{n}}$ & 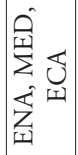 & $\stackrel{\circ}{\mathrm{Z}}$ & 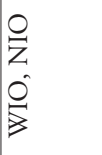 & $\begin{array}{l}0 \\
\stackrel{0}{Z} \\
0 \\
0 \\
3\end{array}$ \\
\hline 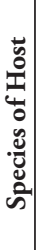 & 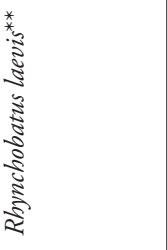 & 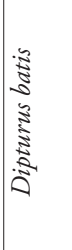 & 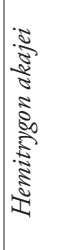 & 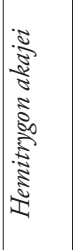 & $\begin{array}{l}\text { ईँ } \\
\text { हूँ } \\
\text { हूँ } \\
\text { हूँ } \\
\text { हूँ } \\
z\end{array}$ & 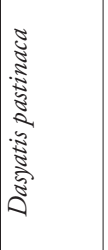 & 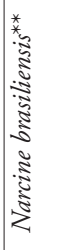 & 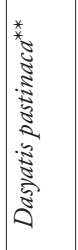 & 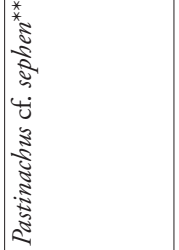 & 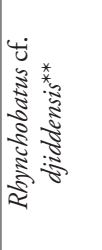 & 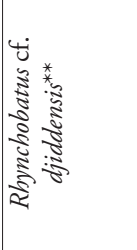 \\
\hline 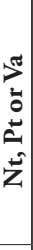 & 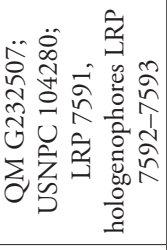 & 孚 & 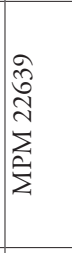 & 孚 & 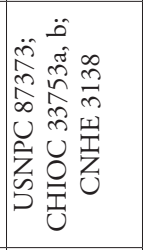 & 㟔 & 点 & 孚 & 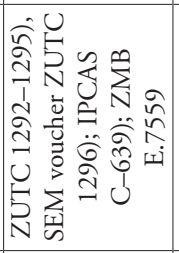 & 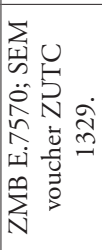 & 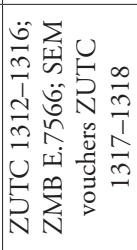 \\
\hline 量 & 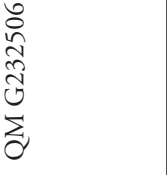 & 点 & 点 & 占 & 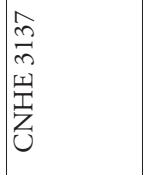 & 孚 & 党 & 孚 & 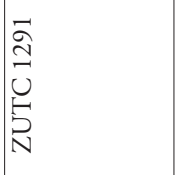 & $\begin{array}{l}\infty \\
\tilde{n} \\
\vdots \\
u \\
\vdots \\
\\
N\end{array}$ & $\begin{array}{l}\exists \\
\vec{n} \\
u \\
\vdots \\
\delta \\
N\end{array}$ \\
\hline 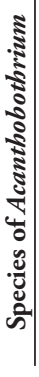 & 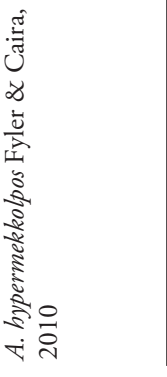 & 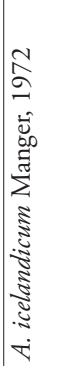 & 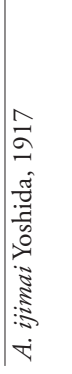 & 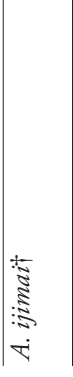 & 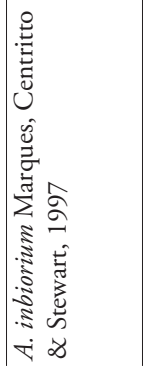 & 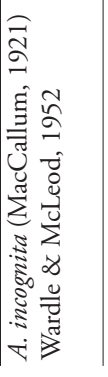 & 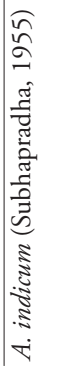 & 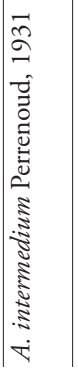 & 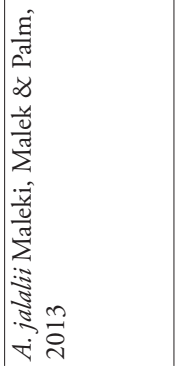 & 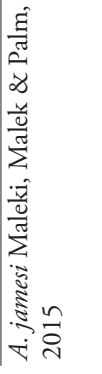 & 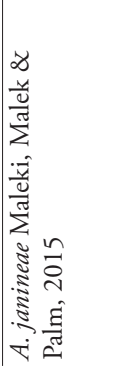 \\
\hline
\end{tabular}




\begin{tabular}{|c|c|c|c|c|c|c|c|}
\hline & 㕺 & 禾 & $=$ & $=$ & $\stackrel{c}{c}$ & $\cong$ & \\
\hline & 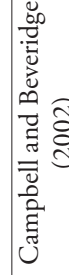 & & 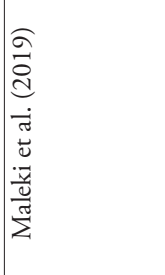 & 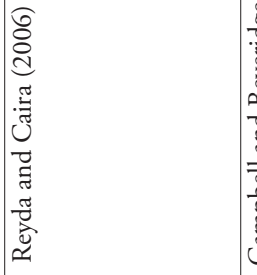 & 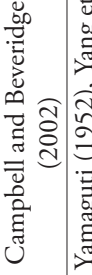 & 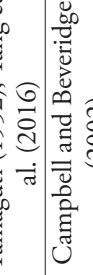 & \\
\hline كُ & 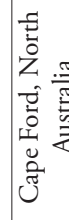 & & 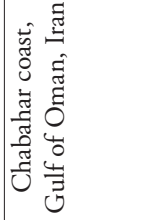 & 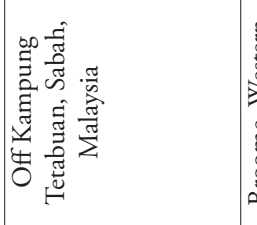 & 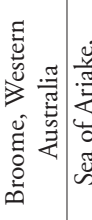 & 离 & \\
\hline 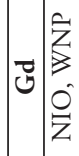 & n. & $\begin{array}{l}\hat{\tilde{z}} \\
\hat{z} \\
\hat{O}^{\prime} \\
\bar{z}\end{array}$ & 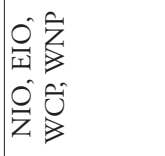 & $\begin{array}{l}\overline{\tilde{z}} \\
\bar{z} \\
\dot{0} \\
\bar{Z}\end{array}$ & 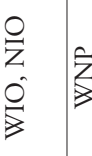 & $\frac{O}{z}$ & \\
\hline 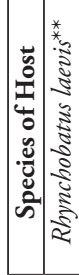 & 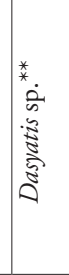 & 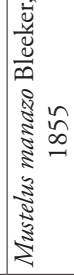 & 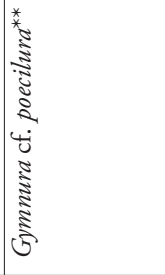 & & 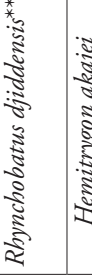 & 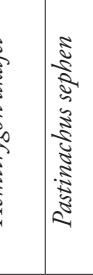 & \\
\hline 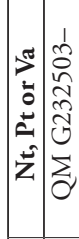 & 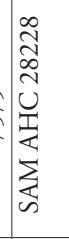 & 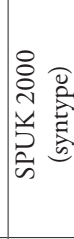 & 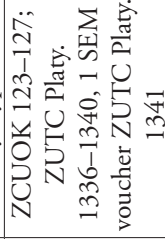 & 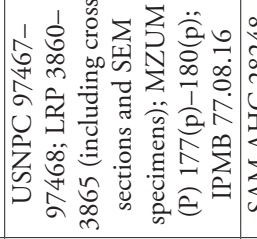 & 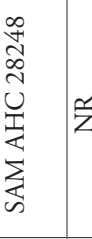 & 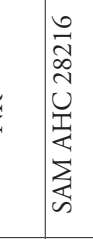 & \\
\hline 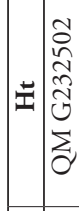 & 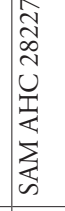 & $\tilde{z}$ & 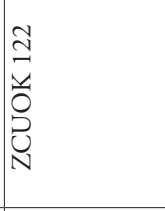 & 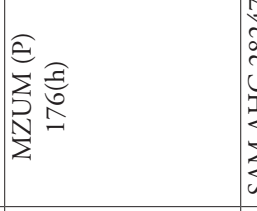 & 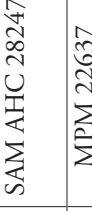 & 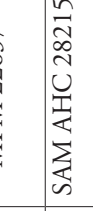 & \\
\hline 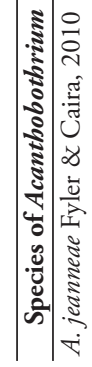 & 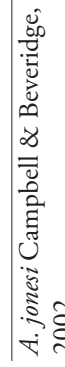 & 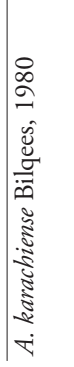 & 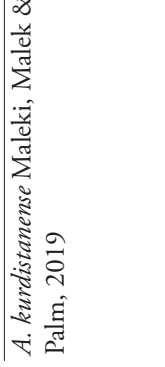 & 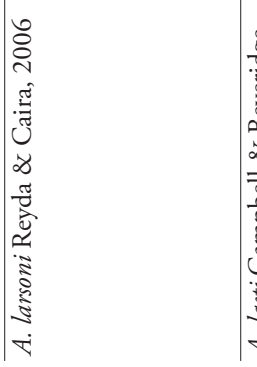 & 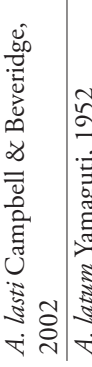 & 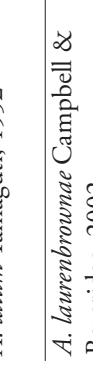 & \\
\hline
\end{tabular}




\begin{tabular}{|c|c|c|c|c|c|c|c|c|c|c|c|c|}
\hline $\bar{J}$ & $\Xi$ & $\approx$ & 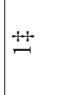 & $\begin{array}{l}\vec{\alpha} \\
\approx \\
\infty \\
\infty \\
\infty\end{array}$ & 1 & : & $\pi$ & క్ర & 导 & 1 & 1 & $\frac{++}{\hat{N}}$ \\
\hline$\ddot{\circ}$ & 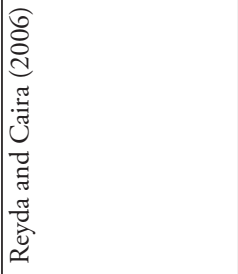 & 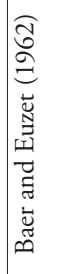 & 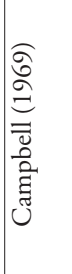 & 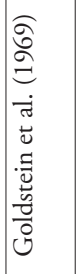 & 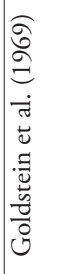 & 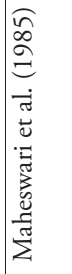 & 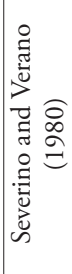 & 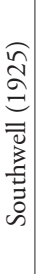 & 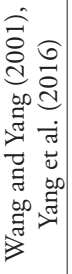 & 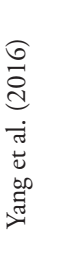 & 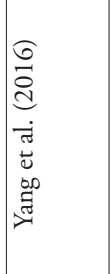 & 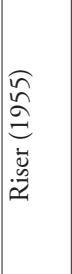 \\
\hline 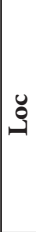 & 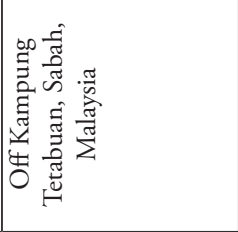 & 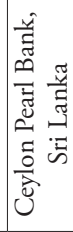 & 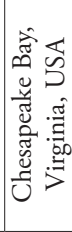 & 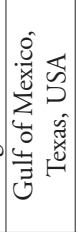 & 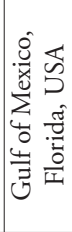 & 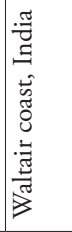 & 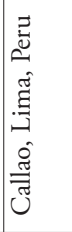 & 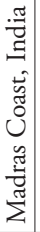 & 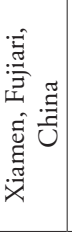 & 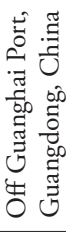 & 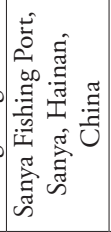 & 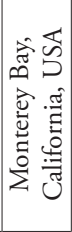 \\
\hline తే & $\begin{array}{l}\tilde{U} \\
\dot{z} \\
\hat{0} \\
\bar{Z}\end{array}$ & n. & 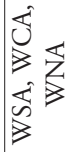 & 吕 & 鹿 & $\begin{array}{ll}0 & 0 \\
Z & 0 \\
0 & 0 \\
0 & 0 \\
3 & =1\end{array}$ & 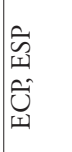 & n. & $\vec{z}$ & $\vec{z}$ & $\stackrel{\tilde{z}}{z}$ & $\begin{array}{l}\hat{U} \\
\text { II } \\
\hat{\bar{Z}} \\
\underline{I I}\end{array}$ \\
\hline 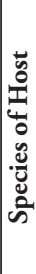 & 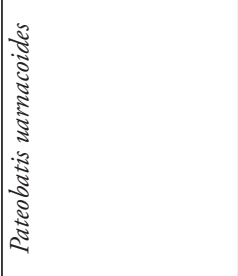 & 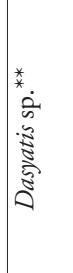 & 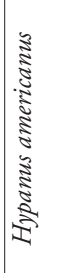 & 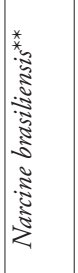 & 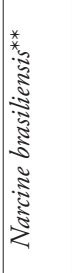 & 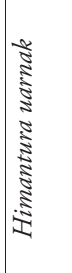 & 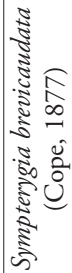 & $\begin{array}{l}* \\
* \\
\dot{2} \\
\hat{2} \\
3 \\
\vdots\end{array}$ & 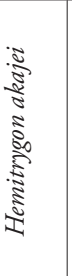 & 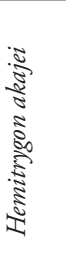 & 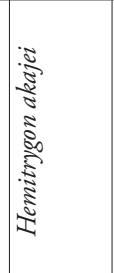 & 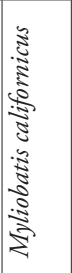 \\
\hline & 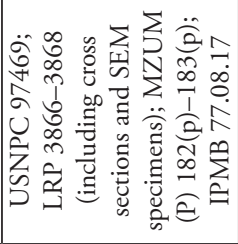 & $\frac{\widetilde{z}}{z}$ & 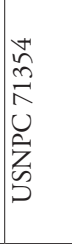 & 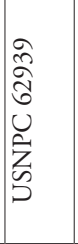 & $\begin{array}{l}\vec{\sim} \\
\infty \\
\hat{N} \\
u \\
\tilde{Z} \\
\tilde{n} \\
\sigma\end{array}$ & 孚 & 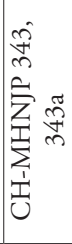 & 点 & 点 & 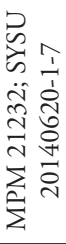 & $\frac{a}{z}$ & 孚 \\
\hline \pm & 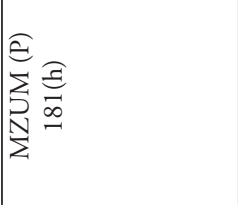 & $\tilde{z}$ & $\begin{array}{l}n \\
\tilde{n} \\
\hat{n} \\
u \\
\tilde{z} \\
\tilde{n}\end{array}$ & 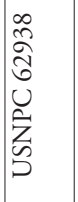 & $\frac{a}{z}$ & 光 & 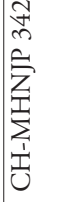 & 孚 & 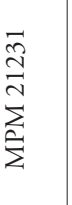 & $\begin{array}{l}\vec{D} \\
\vec{\Xi} \\
\sum \\
\vdots \\
\vdots \\
\Sigma\end{array}$ & 号 & 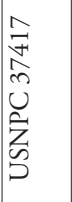 \\
\hline 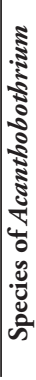 & 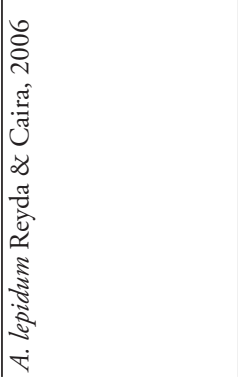 & 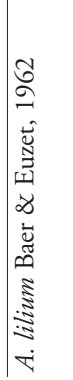 & 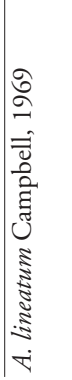 & 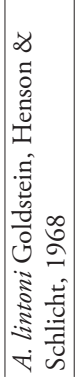 & 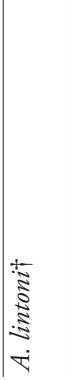 & 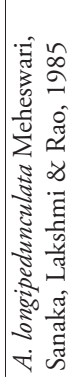 & 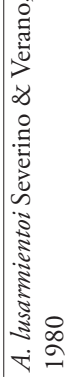 & 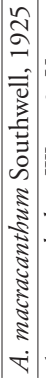 & 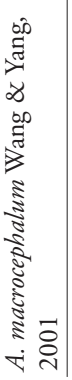 & 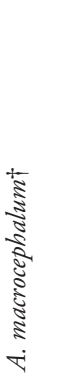 & 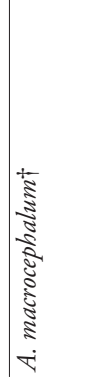 & 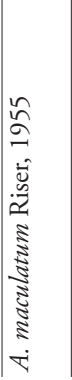 \\
\hline
\end{tabular}




\begin{tabular}{|c|c|c|c|c|c|c|}
\hline & $=$ & in & $\infty$ & $\#$ & $\infty$ & \\
\hline & 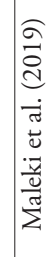 & 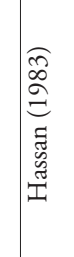 & 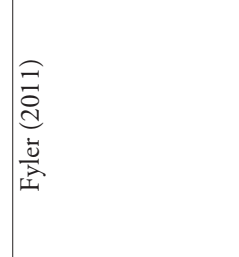 & & 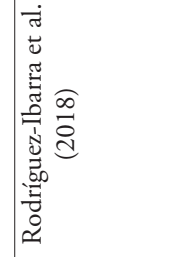 & \\
\hline & 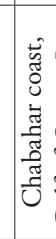 & 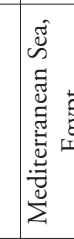 & 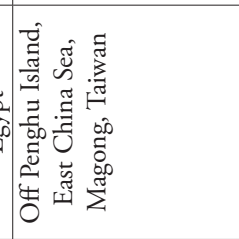 & 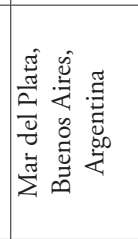 & 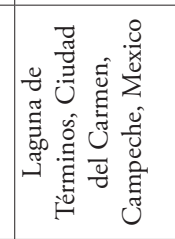 & \\
\hline & 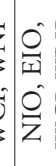 & $\frac{0}{\bar{z}}$ & $\begin{array}{l}\hat{\tilde{E}} \\
\hat{z} \\
\hat{z} \\
\hat{z}\end{array}$ & 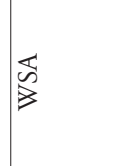 & 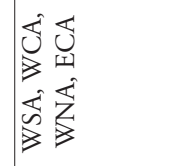 & \\
\hline $\mid$ & 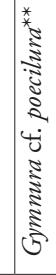 & 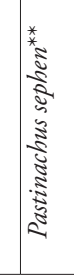 & 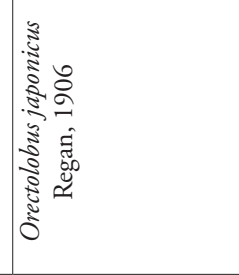 & 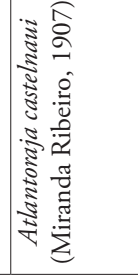 & 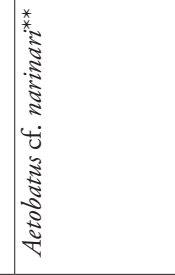 & 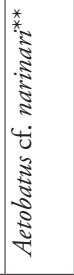 \\
\hline$\left|\begin{array}{|c|c|}\mid \vec{a} \\
\\
\end{array}\right|$ & & 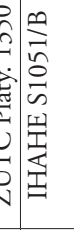 & 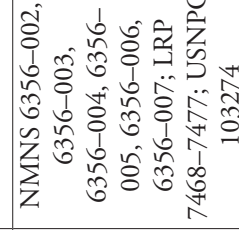 & 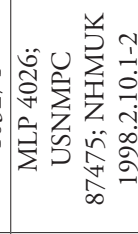 & 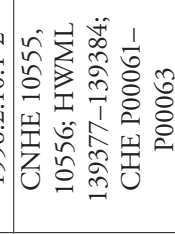 & ż \\
\hline \pm & 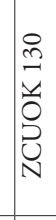 & 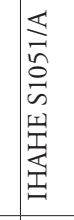 & 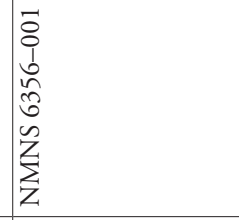 & 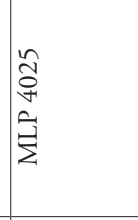 & 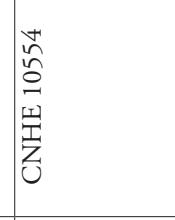 & ź \\
\hline 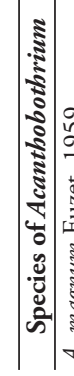 & 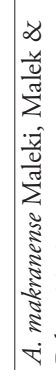 & 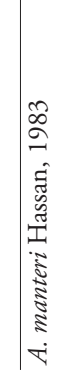 & 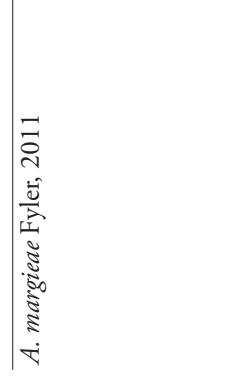 & 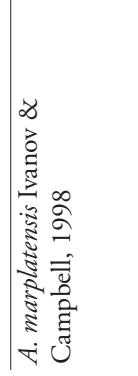 & 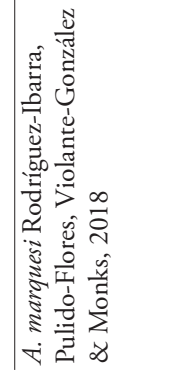 & 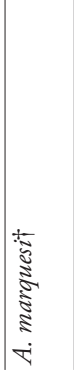 \\
\hline
\end{tabular}




\begin{tabular}{|c|c|c|c|c|c|c|c|c|c|c|}
\hline & $=$ & & $\approx$ & $\cong$ & 1 & $F$ & F & 1 & & 苏 \\
\hline 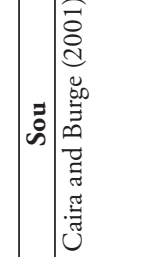 & 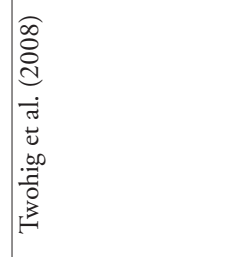 & 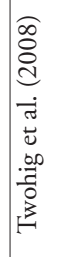 & 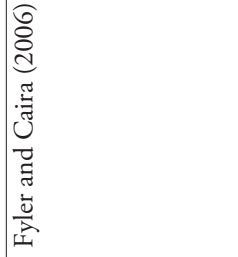 & 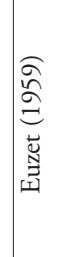 & 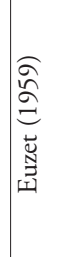 & 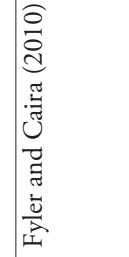 & 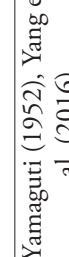 & 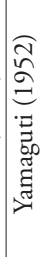 & 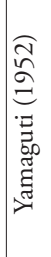 & 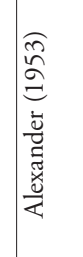 \\
\hline (1) & 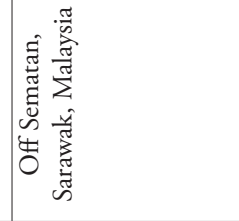 & 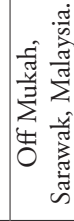 & 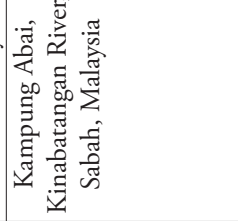 & 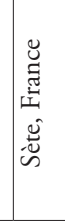 & 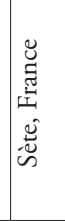 & 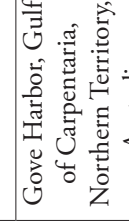 & 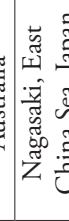 & & 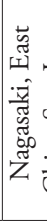 & 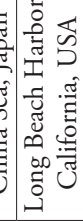 \\
\hline ज़ & $\frac{\rho}{z}$ & $\frac{0}{Z}$ & $\begin{array}{l}\overline{\tilde{y}} \\
\overline{0} \\
\overline{\mathbf{z}}\end{array}$ & 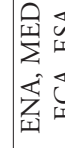 & 舟 & $\begin{array}{l}\hat{z} \\
\hat{z} \\
0 \\
\hat{z}\end{array}$ & 位 & & $\mid \tilde{z}$ & $\begin{array}{l}\hat{\tilde{u}} \\
\text { 竎 } \\
\hat{\mathbf{u}}\end{array}$ \\
\hline 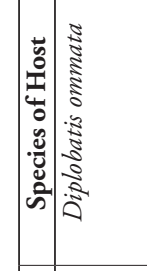 & 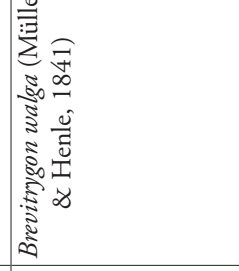 & 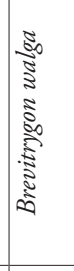 & 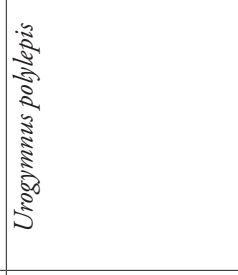 & 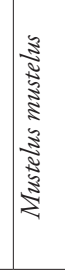 & 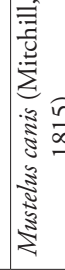 & 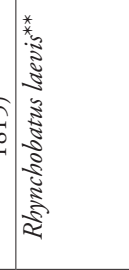 & 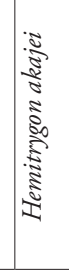 & 苾 & है & 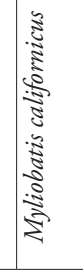 \\
\hline 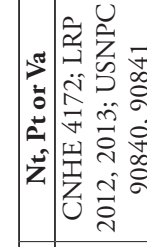 & 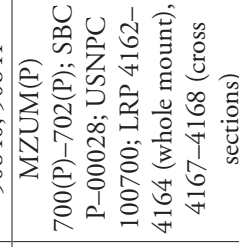 & & 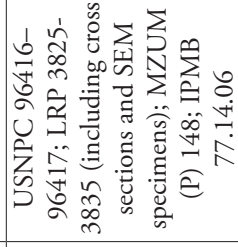 & $\tilde{z}$ & $\tilde{z}$ & 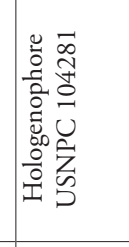 & 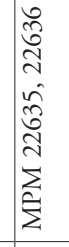 & 苂 & 艺 & $\tilde{z}$ \\
\hline 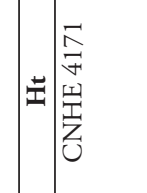 & 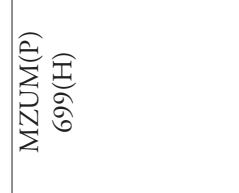 & $\tilde{z}$ & 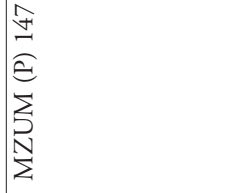 & $\tilde{z}$ & 苂 & 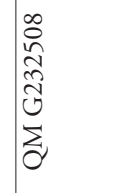 & $\tilde{z}$ & žz & ẑ & $\begin{array}{l}\tilde{n} \\
\infty \\
0 \\
0 \\
\tilde{z} \\
3 \\
0\end{array}$ \\
\hline 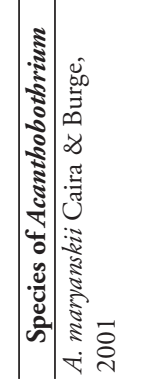 & 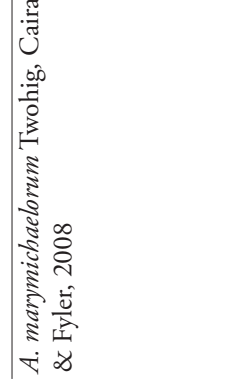 & 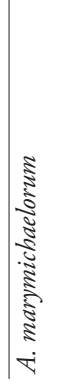 & 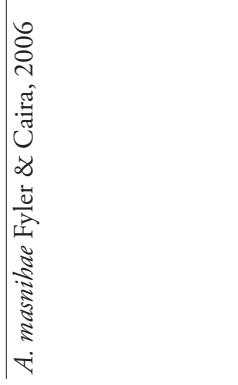 & 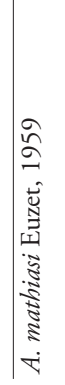 & 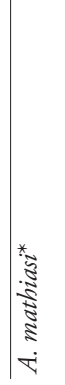 & 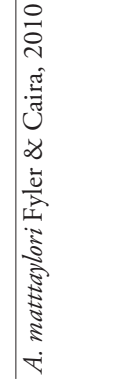 & 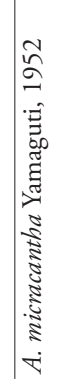 & 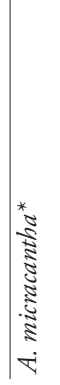 & 恼 & 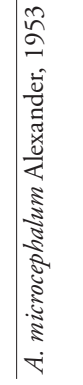 \\
\hline
\end{tabular}




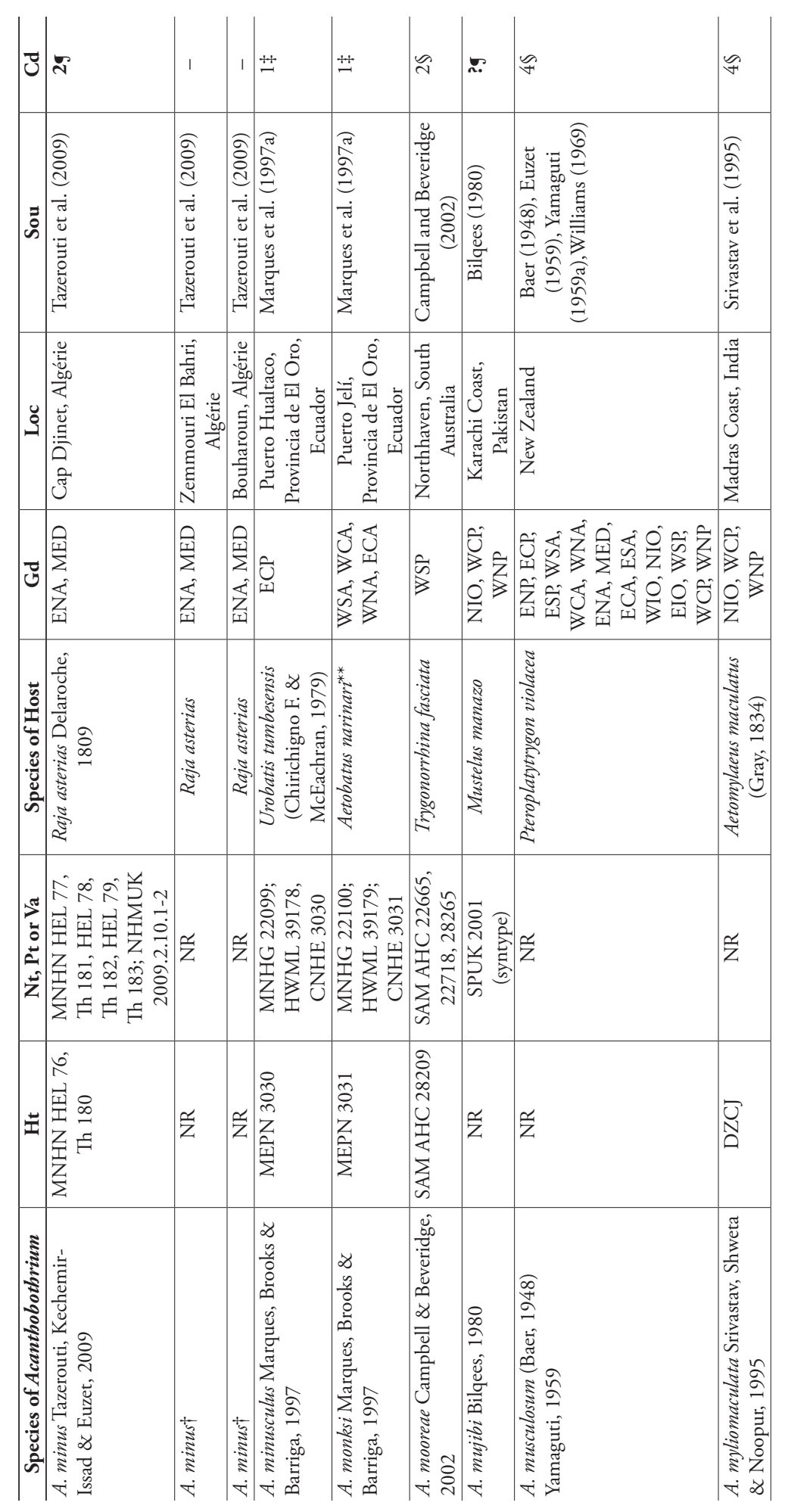




\begin{tabular}{|c|c|c|c|c|c|c|c|c|}
\hline J & 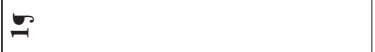 & $\ddot{+}$ & $F$ & 1 & 1 & 1 & 1 & 苧 \\
\hline$\ddot{\circ}$ & 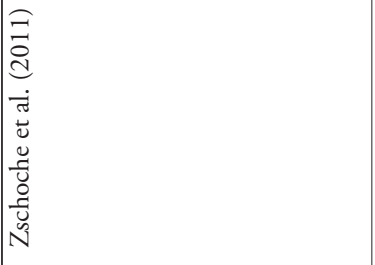 & 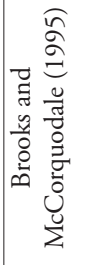 & 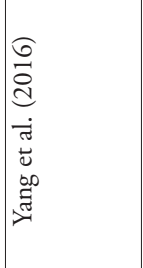 & 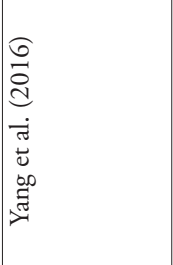 & 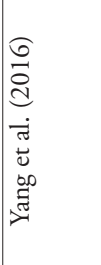 & 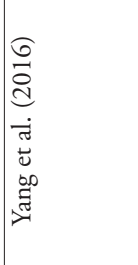 & 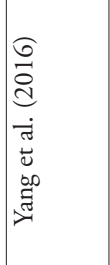 & 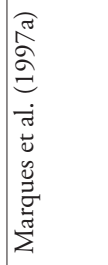 \\
\hline 岁 & 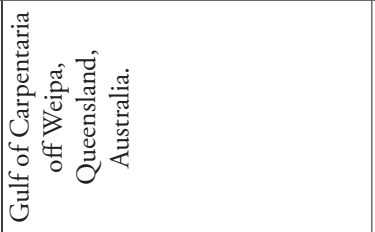 & 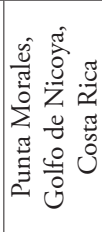 & 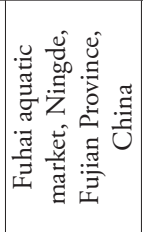 & 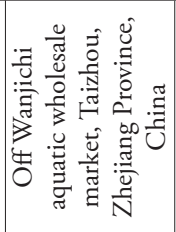 & 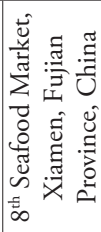 & 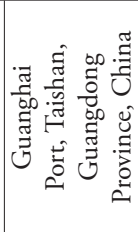 & 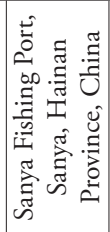 & 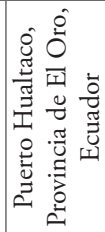 \\
\hline & 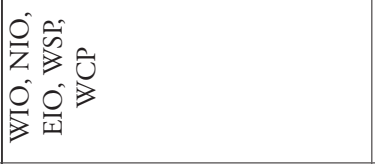 & 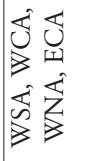 & $\hat{z}$ & $\hat{z}$ & $\vec{z}$ & $\hat{z}$ & $\vec{z}$ & 仓્| \\
\hline 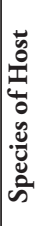 & 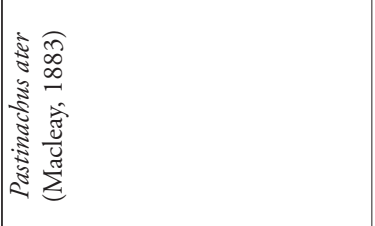 & 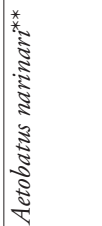 & 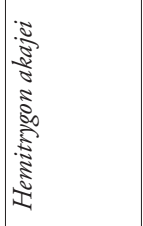 & 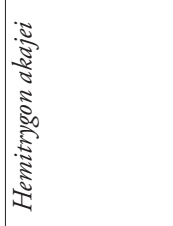 & 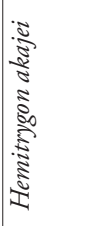 & 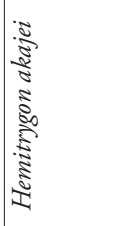 & 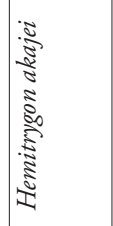 & $\begin{array}{l}\sqrt{4} \\
5 \\
3 \\
3 \\
3 \\
3 \\
3 \\
3 \\
3\end{array}$ \\
\hline & 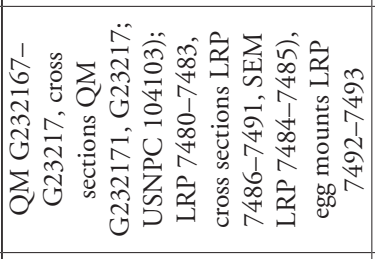 & 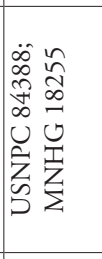 & 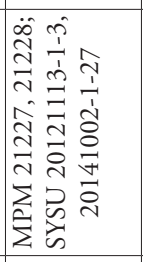 & $\tilde{z}$ & 觉 & 号 & 孚 & 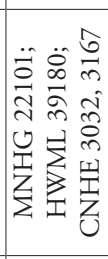 \\
\hline & 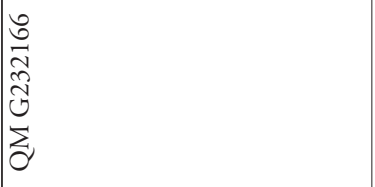 & 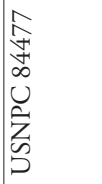 & 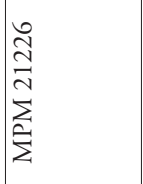 & $\tilde{z}$ & 学 & $\tilde{z}$ & 学 & $\begin{array}{l}\tilde{o} \\
\tilde{n} \\
z \\
\vdots \overline{i=1} \\
\sum\end{array}$ \\
\hline 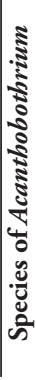 & 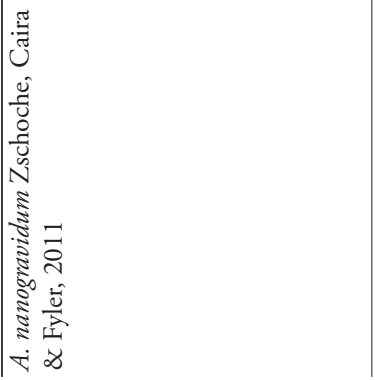 & 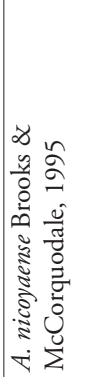 & 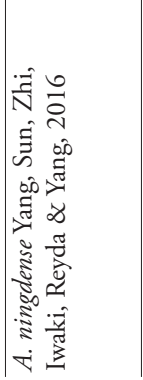 & 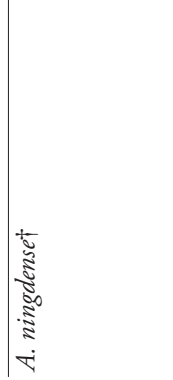 & 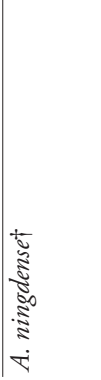 & 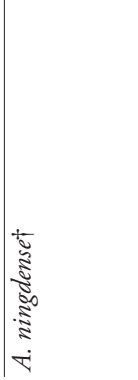 & 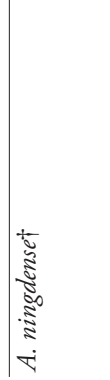 & 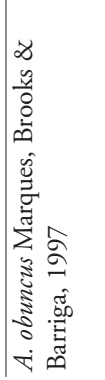 \\
\hline
\end{tabular}




\begin{tabular}{|c|c|c|c|c|c|c|c|c|c|c|}
\hline 4 & $=$ & $\cong$ & 1 & & 1 & 1 & 1 & $=$ & 1 & 茫 \\
\hline 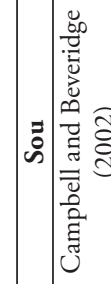 & 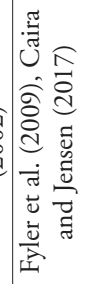 & 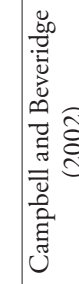 & 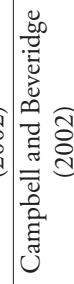 & 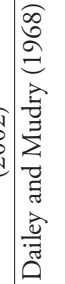 & 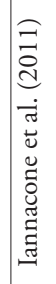 & 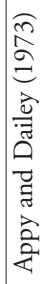 & 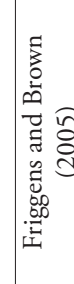 & 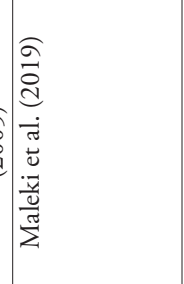 & 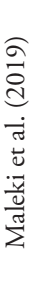 & \\
\hline 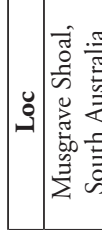 & 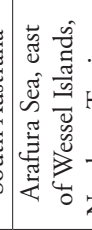 & 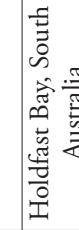 & 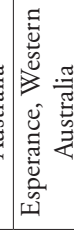 & 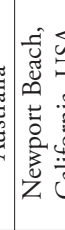 & 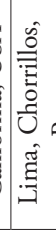 & 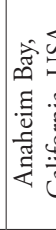 & 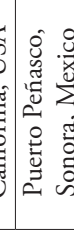 & 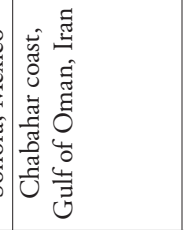 & 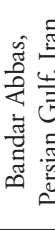 & 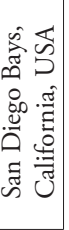 \\
\hline تص & $\begin{array}{l}\hat{\tilde{y}} \\
\hat{\beta} \\
\hat{\tilde{y}} \\
\hat{z}\end{array}$ & $\frac{0}{\pi}$ & $\frac{0}{i n}$ & $\begin{array}{l}\tilde{\tilde{u}} \\
\tilde{u} \\
\tilde{\tilde{n}}\end{array}$ & $\begin{array}{l}\hat{\tilde{y}} \\
\hat{i} \\
\hat{y} \\
\hat{H}\end{array}$ & $\begin{array}{l}\text { 谓 } \\
\hat{\tilde{z}} \\
\text { in }\end{array}$ & $\begin{array}{l}\hat{\tilde{u}} \\
\hat{\mathbf{z}} \\
\hat{\mathbf{y}}\end{array}$ & 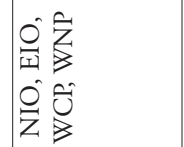 & & 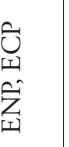 \\
\hline 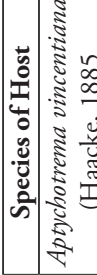 & 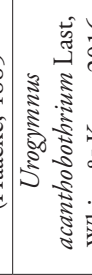 & 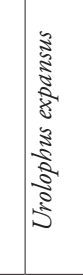 & 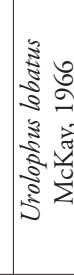 & 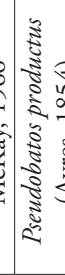 & 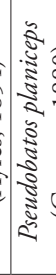 & 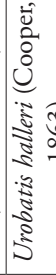 & 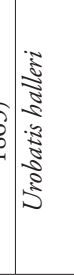 & 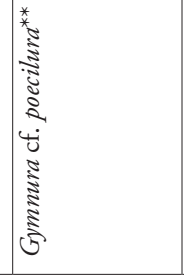 & 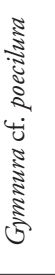 & \\
\hline 芯 & 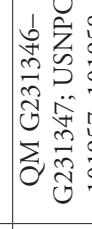 & $\mid$ & $\tilde{z}$ & $\tilde{z}$ & $\tilde{z}$ & ż & $\tilde{z}$ & 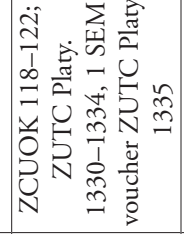 & 艺 & 苂 \\
\hline 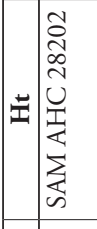 & 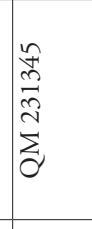 & 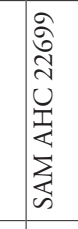 & $\tilde{z}$ & 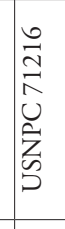 & 苂 & ż & $\tilde{z}$ & 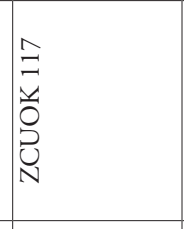 & 乩 & 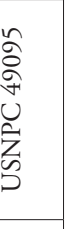 \\
\hline 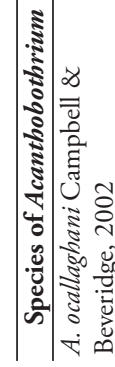 & 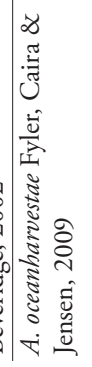 & 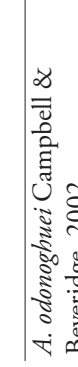 & 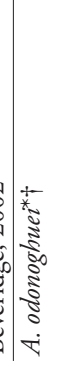 & हू & 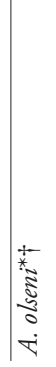 & 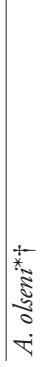 & 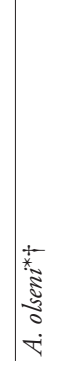 & 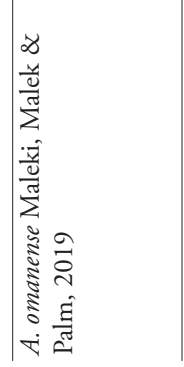 & 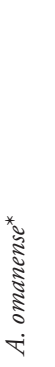 & 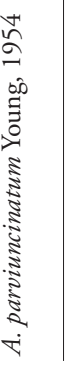 \\
\hline
\end{tabular}




\begin{tabular}{|c|c|c|c|c|c|c|c|c|c|c|c|}
\hline $\bar{U}$ & I & 1 & $\approx$ & $\begin{array}{l}++ \\
\hat{N} \\
\curvearrowright \\
\infty \\
\infty \\
=\end{array}$ & 1 & 1 & $\cong$ & $=$ & 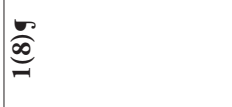 & \&্ণ & 1 \\
\hline के & 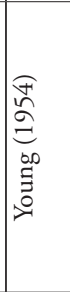 & 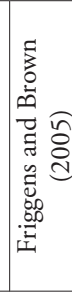 & 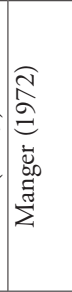 & 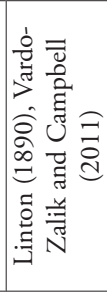 & 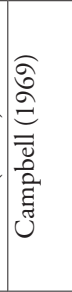 & 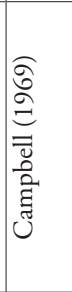 & 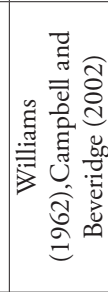 & 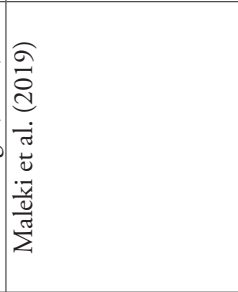 & 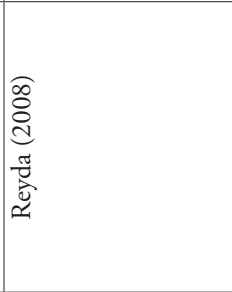 & 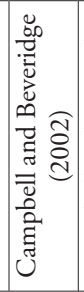 & 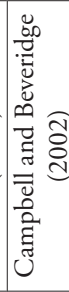 \\
\hline ـ & 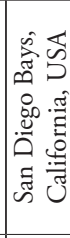 & 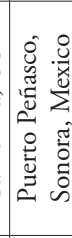 & 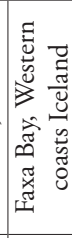 & 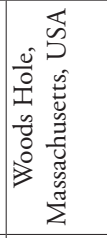 & 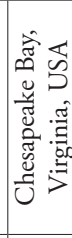 & 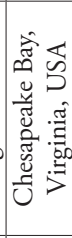 & 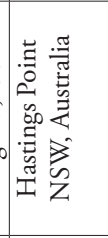 & 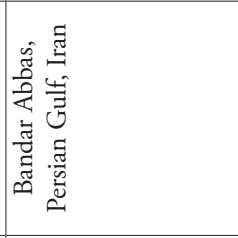 & 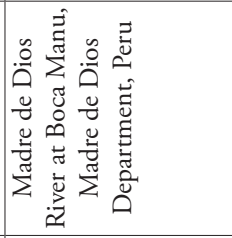 & 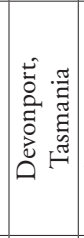 & 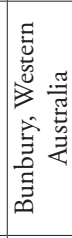 \\
\hline כૈ & U్ & $\begin{array}{l}\hat{U} \\
\text { II } \\
\hat{Z} \\
\dot{Z}\end{array}$ & 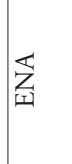 & 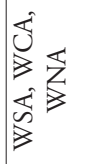 & $\begin{array}{l}\mathbb{z} \\
3 \\
3\end{array}$ & 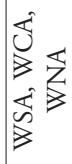 & $\begin{array}{l}\overrightarrow{5} \\
0 \\
0 \\
0 \\
1\end{array}$ & 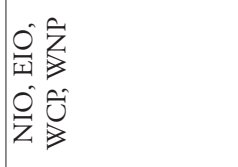 & 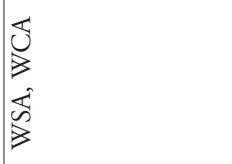 & $\begin{array}{l}\tilde{n} \\
\tilde{B} \\
0 \\
0 \\
0=1\end{array}$ & $\begin{array}{l}\tilde{\tilde{n}} \\
\hat{\nabla} \\
0 \\
0 \\
0.1\end{array}$ \\
\hline 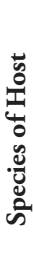 & 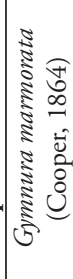 & 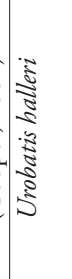 & 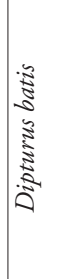 & 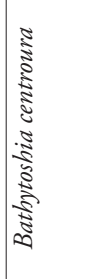 & 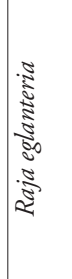 & 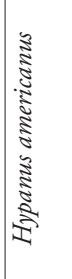 & 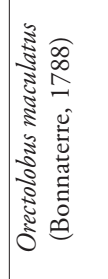 & 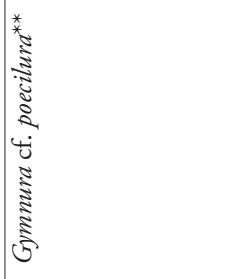 & 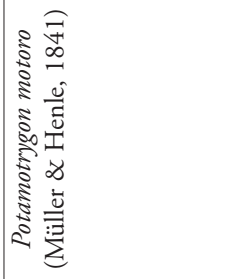 & 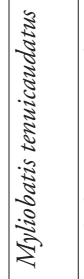 & 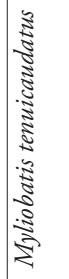 \\
\hline مَّ & 孚 & 光 & 觉 & 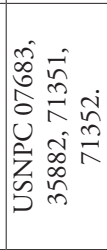 & 学 & 孚 & 孚 & 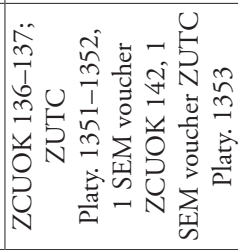 & 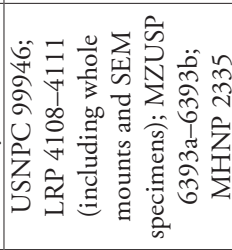 & 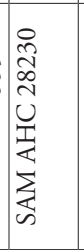 & 号 \\
\hline \pm & 孚 & 㟔 & $\tilde{z}$ & $\tilde{z}$ & $\frac{\tilde{z}}{z}$ & $\tilde{z}$ & 孚 & 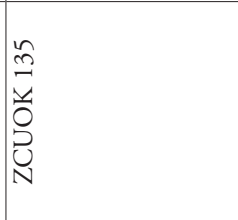 & $\begin{array}{l}\tilde{a} \\
\hat{\alpha} \\
\alpha \\
0 \\
\hat{z} \\
\tilde{n} \\
\partial\end{array}$ & 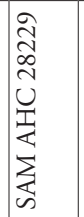 & $\frac{\widetilde{z}}{z}$ \\
\hline 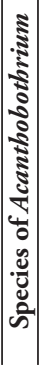 & 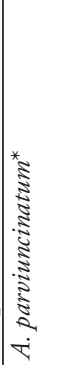 & 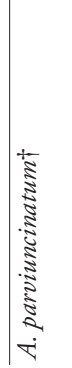 & 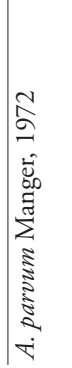 & 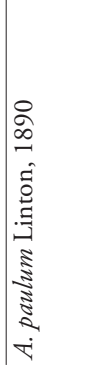 & 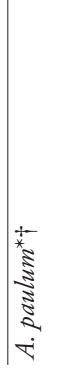 & 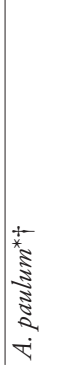 & 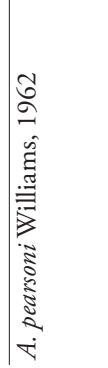 & 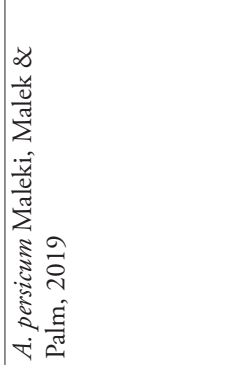 & 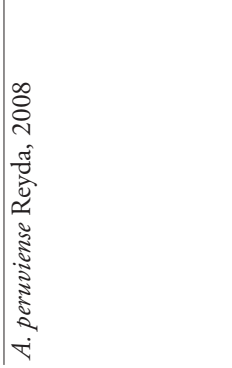 & 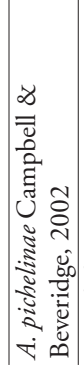 & 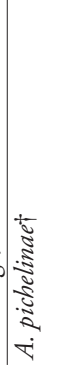 \\
\hline
\end{tabular}




\begin{tabular}{|c|c|c|c|c|c|c|c|c|c|c|c|}
\hline उٓ & $F$ & $\underset{\sigma}{\mathscr{N}}$ & s. & 1 & $\mathbb{4}$ & $\underset{N}{ }$ & 获 & 1 & $F$ & $\stackrel{+H}{\sim}$ & $\mathscr{\sim}$ \\
\hline$\ddot{\Xi}$ & 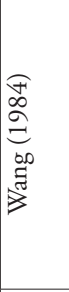 & 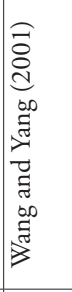 & 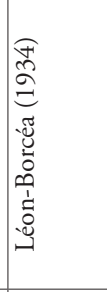 & 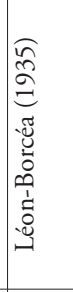 & 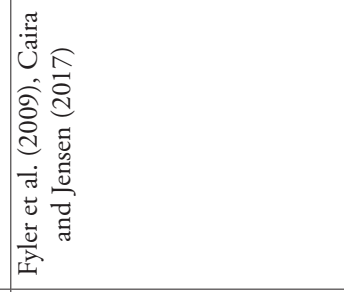 & 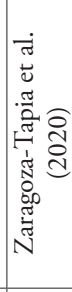 & 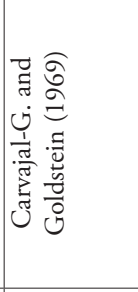 & 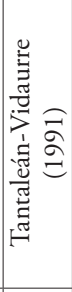 & 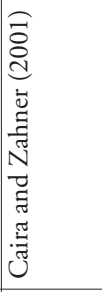 & 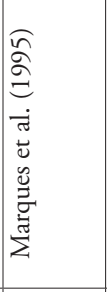 & 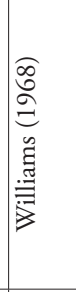 \\
\hline 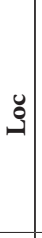 & 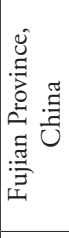 & 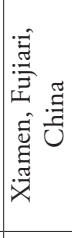 & 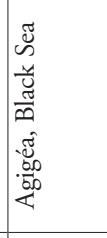 & 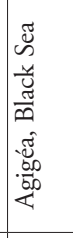 & 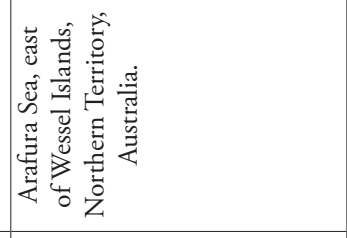 & 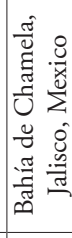 & 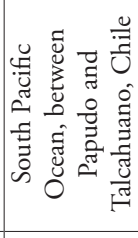 & 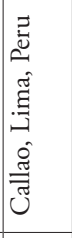 & 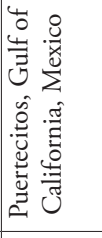 & 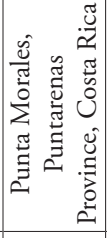 & 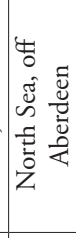 \\
\hline$\vec{\sigma}$ & $\vec{\omega}$ & $n$. & 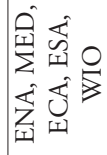 & 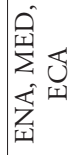 & $\begin{array}{l}\hat{0} \\
3 \\
\tilde{n} \\
\hat{z}\end{array}$ & 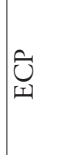 & $\mid \begin{array}{l}\hat{\tilde{n}} \\
\text { II }\end{array}$ & $\begin{array}{l}\hat{\tilde{\omega}} \\
\text { in } \\
\hat{\tilde{E}} \\
\dot{y=1}\end{array}$ & $\begin{array}{l}\hat{w} \\
\text { in } \\
\hat{\tilde{S}} \\
\text { II }\end{array}$ & 论 & 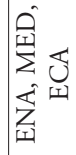 \\
\hline & 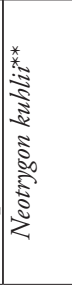 & 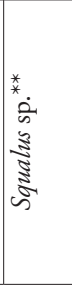 & 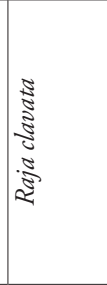 & 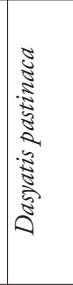 & 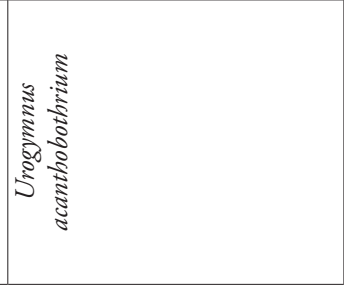 & 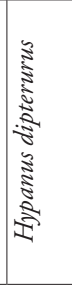 & 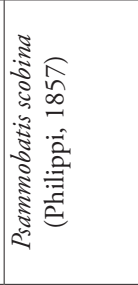 & 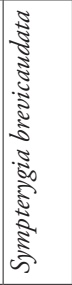 & 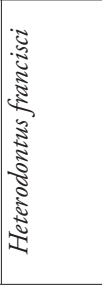 & 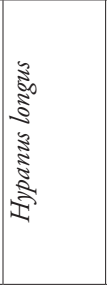 & 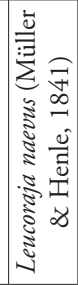 \\
\hline 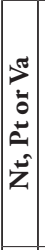 & 号 & 岁 & $\frac{\tilde{z}}{z}$ & $\frac{a}{z}$ & 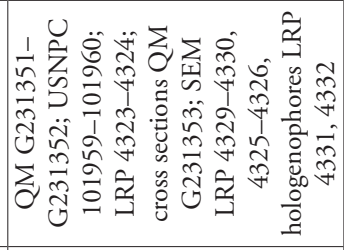 & 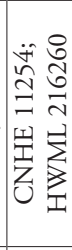 & $\begin{array}{l}\infty \\
\infty \\
n \\
\hat{n} \\
u \\
\tilde{z} \\
\tilde{n} \\
D\end{array}$ & 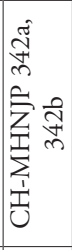 & 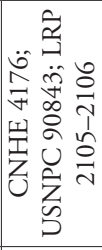 & 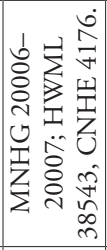 & 号 \\
\hline $\mathbf{\pm}$ & 觉 & 晃 & $\tilde{z}$ & 觉 & $\begin{array}{l}\stackrel{0}{n} \\
\tilde{n} \\
\tilde{n} \\
\tilde{d} \\
\sum\end{array}$ & 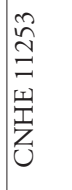 & $\begin{array}{l}\hat{n} \\
\tilde{n} \\
\hat{n} \\
u \\
\hat{z} \\
\sigma\end{array}$ & 孚 & 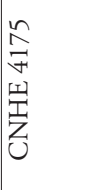 & 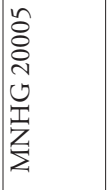 & 号 \\
\hline 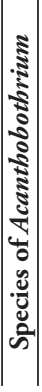 & 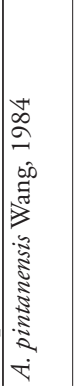 & 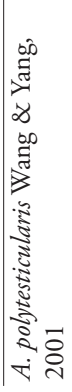 & 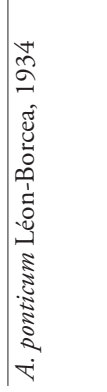 & 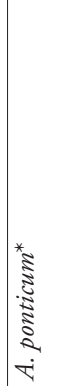 & 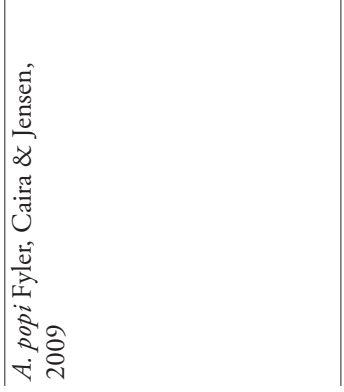 & 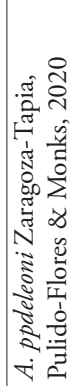 & 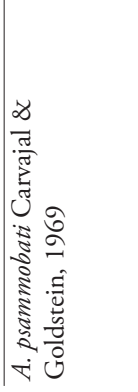 & 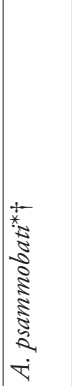 & 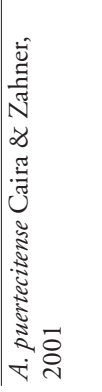 & 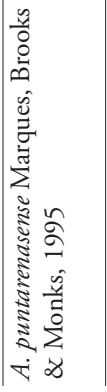 & 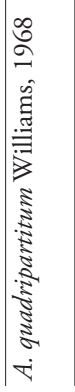 \\
\hline
\end{tabular}




\begin{tabular}{|c|c|c|c|c|c|c|c|c|c|c|c|}
\hline उ & 莎 & 1 & $\approx$ & 1 & 1 & 1 & \begin{tabular}{|l|l|l|}
1 & 1
\end{tabular} & $\stackrel{+}{\sim}$ & $\$$ & 1 & in \\
\hline$\ddot{b}$ & 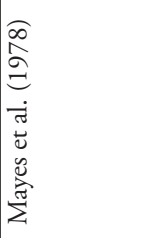 & 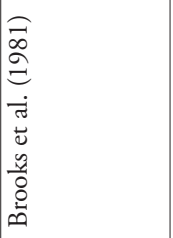 & 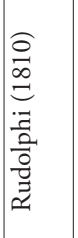 & 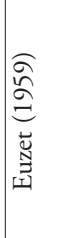 & 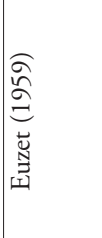 & 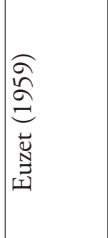 & 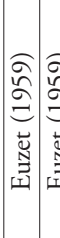 & 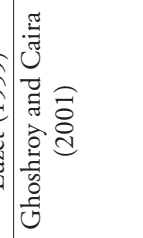 & 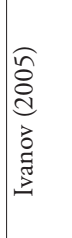 & 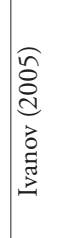 & 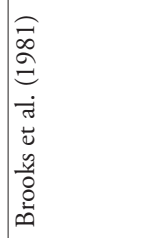 \\
\hline : & 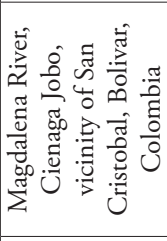 & 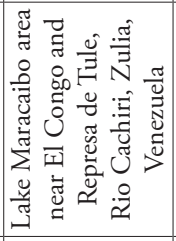 & 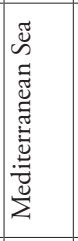 & 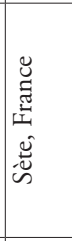 & 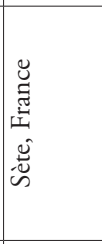 & 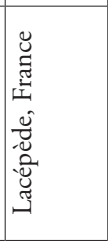 & 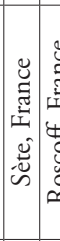 & 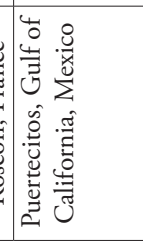 & 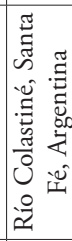 & 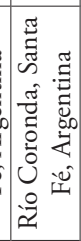 & 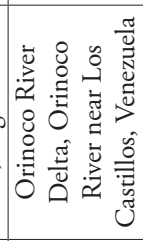 \\
\hline సే & एँ & 岁 & $\overleftrightarrow{z_{i}}$ & 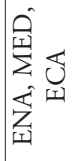 & 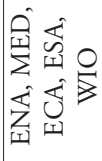 & 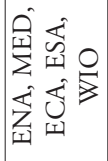 & & $\tilde{U}$ & \begin{tabular}{l}
$\mathbb{3}$ \\
3 \\
\multirow{3}{3}{} \\
3
\end{tabular} & 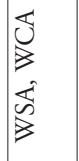 & 芯 \\
\hline 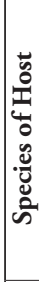 & 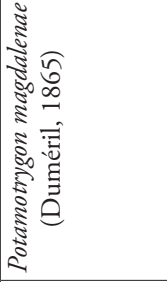 & 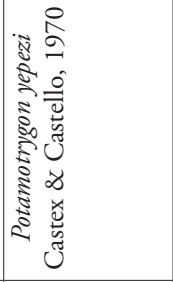 & 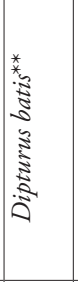 & 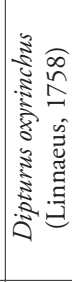 & 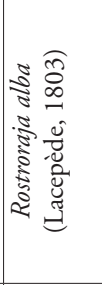 & 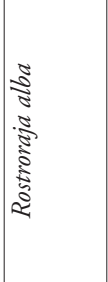 & 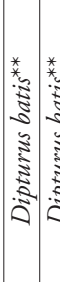 & 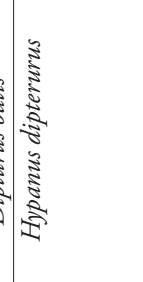 & 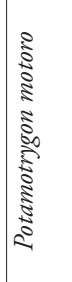 & 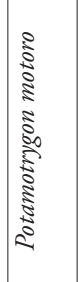 & 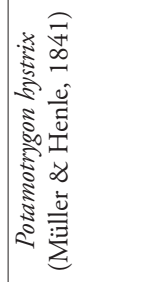 \\
\hline $\mid$ & 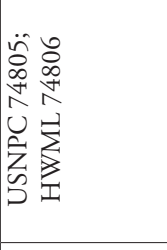 & 号 & 孚 & 号 & 号 & 点 & 点 & 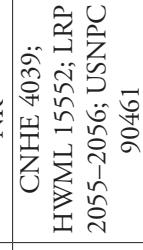 & 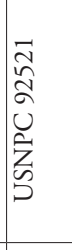 & 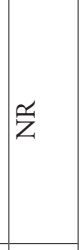 & 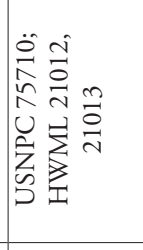 \\
\hline 在 & 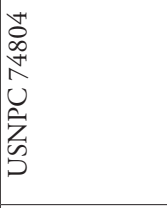 & 岂 & 号 & 号 & 孚 & 岂 & 孚 & 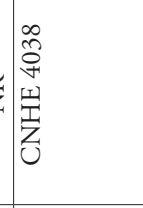 & 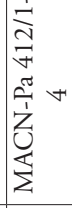 & 点 & $\begin{array}{l}\hat{2} \\
\hat{n} \\
\hat{n} \\
\hat{z} \\
\hat{z} \\
\vdots \\
\delta\end{array}$ \\
\hline 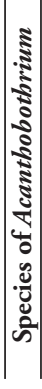 & 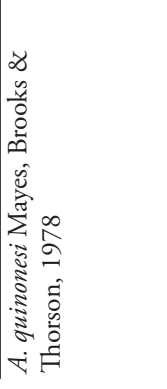 & 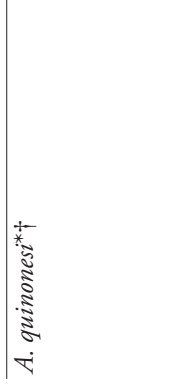 & 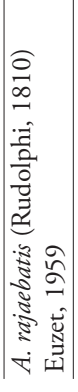 & 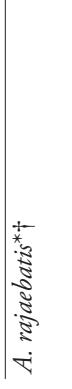 & $\mid$ & 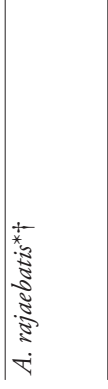 & 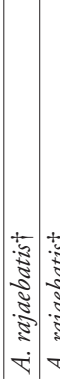 & 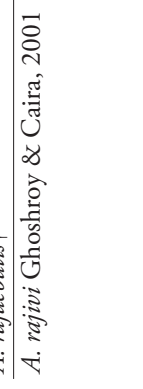 & 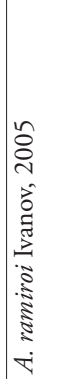 & 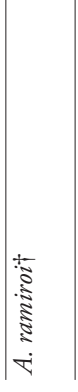 & 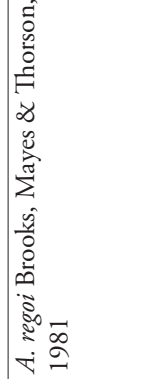 \\
\hline
\end{tabular}




\begin{tabular}{|c|c|c|c|c|c|c|c|c|c|c|c|c|c|c|c|c|c|}
\hline & I & $\left.1\right|^{+t}$ & $\frac{+H}{\sqrt{n}}$ & 1 & $\ddot{m}$ & 1 & 1 & 1 & 1 & 1 & 1 & 1 & I & 1 & 1 & 1 & 1 \\
\hline & 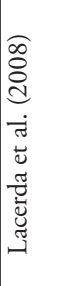 & 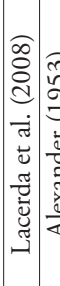 & 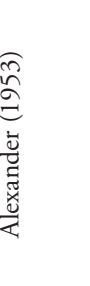 & 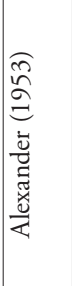 & 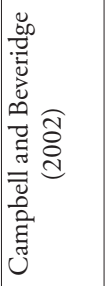 & 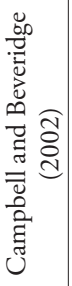 & 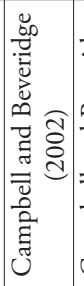 & 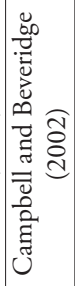 & 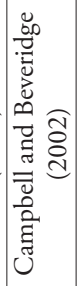 & 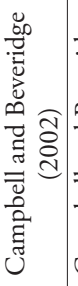 & 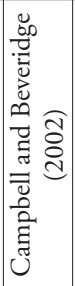 & 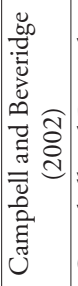 & 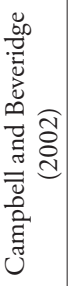 & 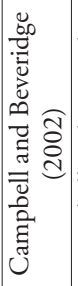 & 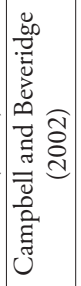 & 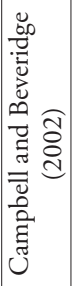 & 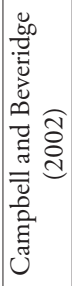 \\
\hline 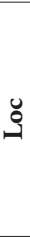 & 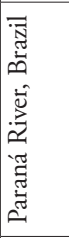 & 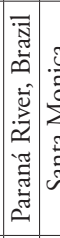 & 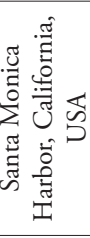 & 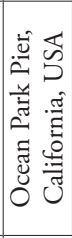 & 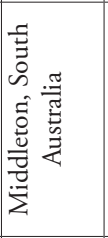 & 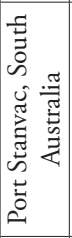 & 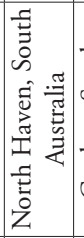 & 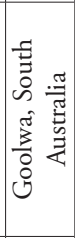 & 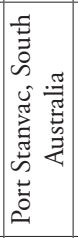 & 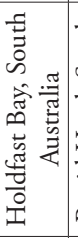 & 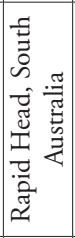 & 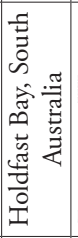 & 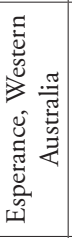 & 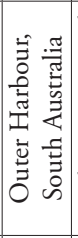 & 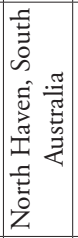 & 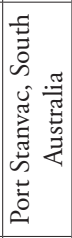 & 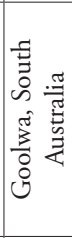 \\
\hline ज & 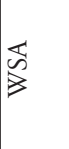 & 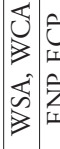 & 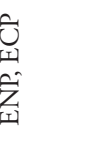 & 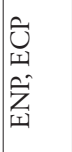 & $\tilde{\tilde{n}}$ & $\begin{array}{l}\overrightarrow{\tilde{y}} \\
\dot{0} \\
\dot{0}\end{array}$ & $\underset{1}{\stackrel{0}{\mid c}}$ & $\underset{|c|}{0}$ & $\begin{array}{l}\hat{\tilde{n}} \\
\vdots \\
0 \\
0 \\
\dot{0}\end{array}$ & $\begin{array}{l}\hat{\tilde{n}} \\
\dot{0} \\
\dot{0}\end{array}$ & 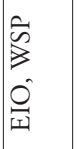 & $\underset{1}{0}$ & $\underset{1}{0}$ & $\tilde{\tilde{\omega}}$ & $\tilde{\tilde{n}}$ & $\tilde{\tilde{n}}$ & $\overrightarrow{\tilde{w}}$ \\
\hline 온 & 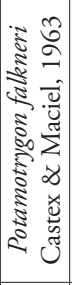 & 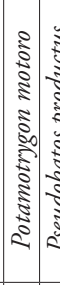 & 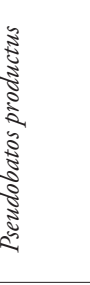 & 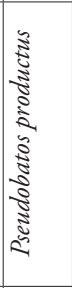 & 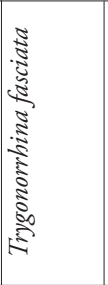 & 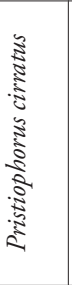 & 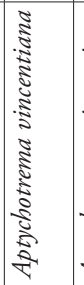 & 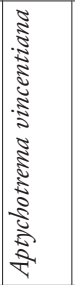 & 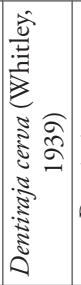 & 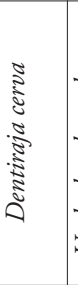 & 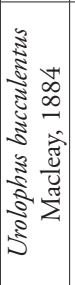 & 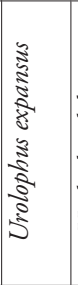 & $\begin{array}{l}\frac{5}{5} \\
\frac{2}{3} \\
\frac{3}{3} \\
\frac{3}{3} \\
3\end{array}$ & 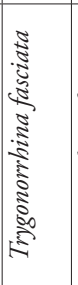 & 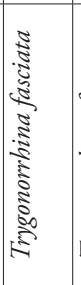 & 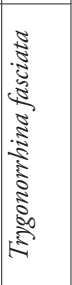 & 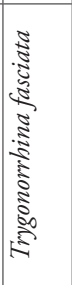 \\
\hline $\begin{array}{l}5 \\
5 \\
5 \\
5 \\
5 \\
5\end{array}$ & 孚 & 点 & 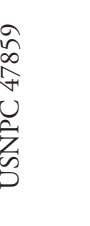 & 孚 & 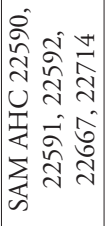 & 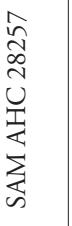 & 吕 & 孚 & 孚 & 岂 & 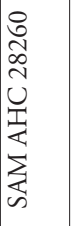 & 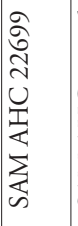 & 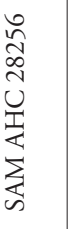 & 号 & 岩 & 岂 & 岂 \\
\hline & & & & & $\sigma$ & & & & & & & & & & & & \\
\hline
\end{tabular}

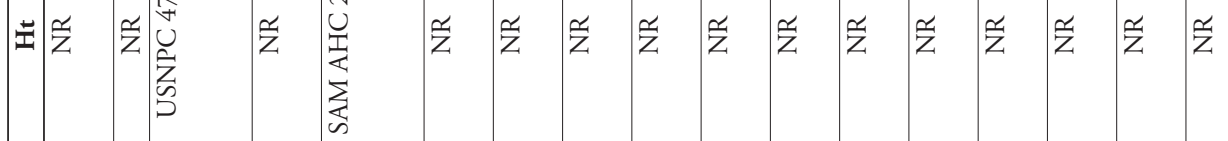
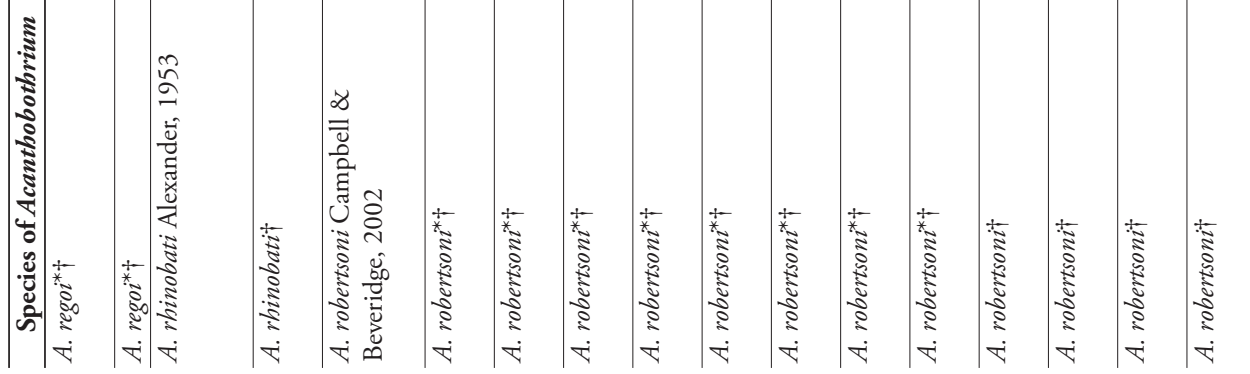


\begin{tabular}{|c|c|c|c|c|c|c|c|c|}
\hline$\vec{\jmath}$ & 1 & 1 & 世 & 1 & 5 & $\cong$ & $=$ & 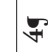 \\
\hline$\vec{b}$ & 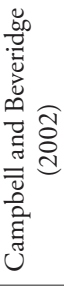 & 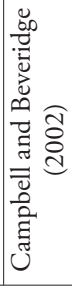 & 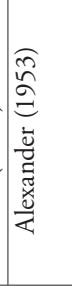 & 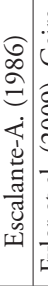 & 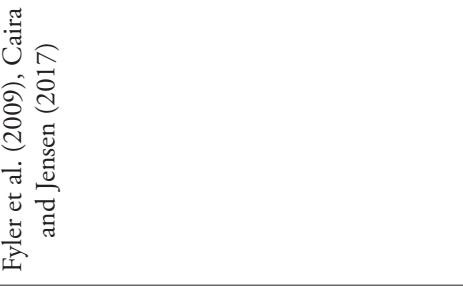 & 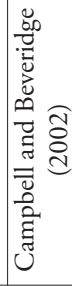 & 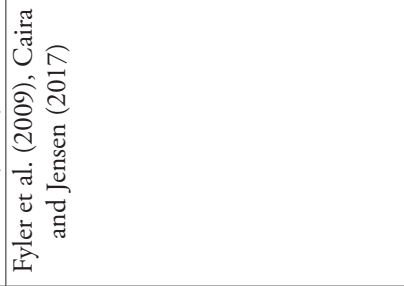 & 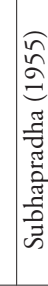 \\
\hline 苞 & 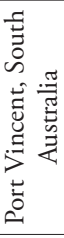 & 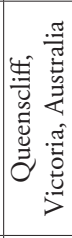 & 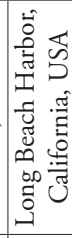 & 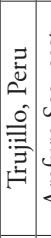 & 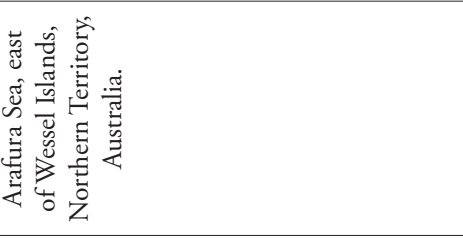 & 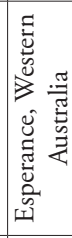 & 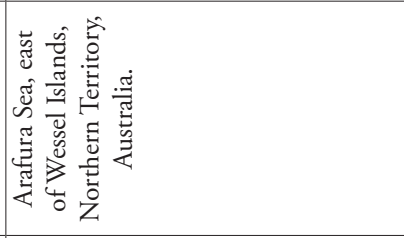 & 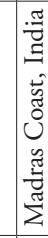 \\
\hline ت & है & $\tilde{n}$ & $\begin{array}{l}\tilde{U} \\
\dot{y} \\
\hat{Z} \\
\hat{Z}\end{array}$ & 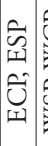 & $\begin{array}{l}\hat{\vec{v}} \\
\hat{\vec{w}} \\
\hat{\nabla}\end{array}$ & $\underset{1}{0}$ & $\begin{array}{l}\hat{U} \\
\hat{z} \\
\tilde{\tilde{n}} \\
\tilde{z}\end{array}$ & $\begin{array}{l}0 \\
Z \\
0 \\
0 \\
3\end{array}$ \\
\hline 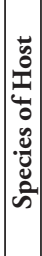 & 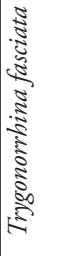 & 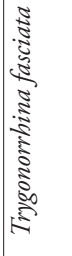 & 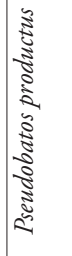 & 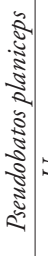 & 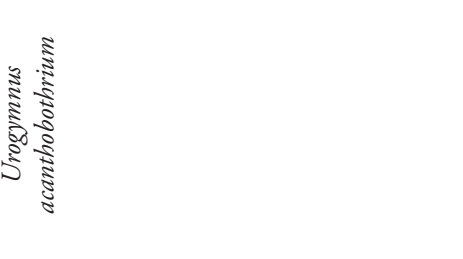 & 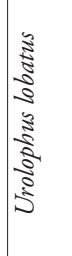 & 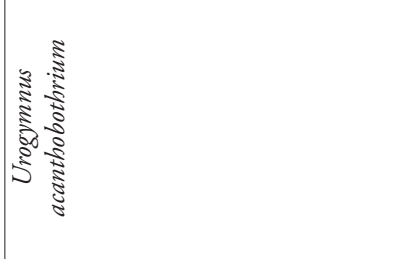 & 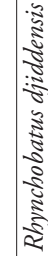 \\
\hline 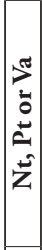 & 品 & 点 & 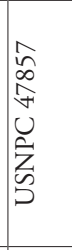 & 光 & 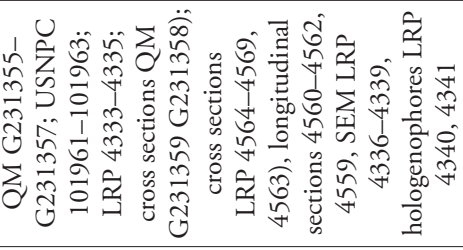 & 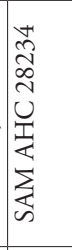 & 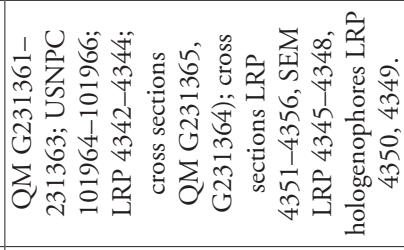 & 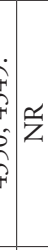 \\
\hline $\mathbf{\pm}$ & 孚 & 点 & 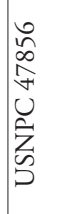 & $\frac{a}{z}$ & 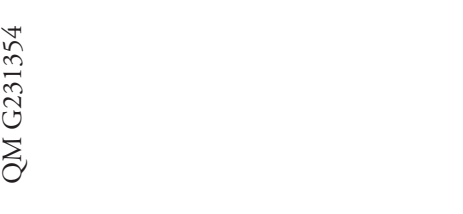 & 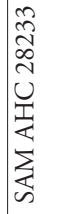 & 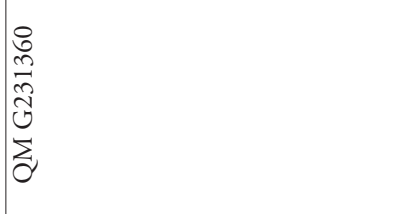 & $\frac{a}{Z}$ \\
\hline 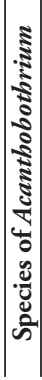 & 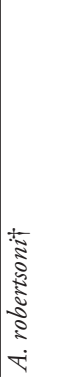 & 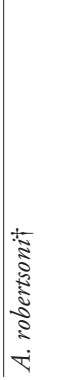 & 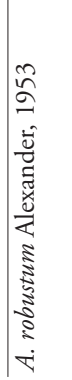 & 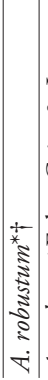 & 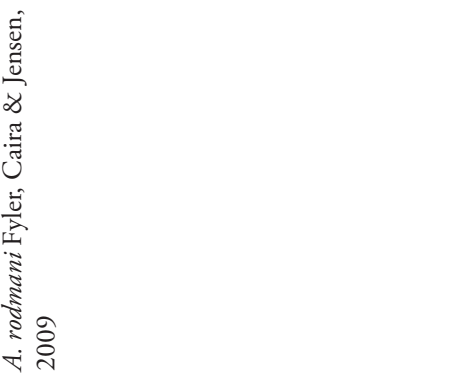 & 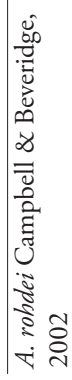 & 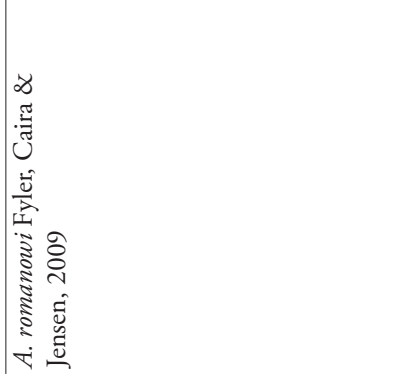 & 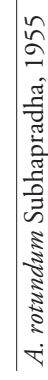 \\
\hline
\end{tabular}




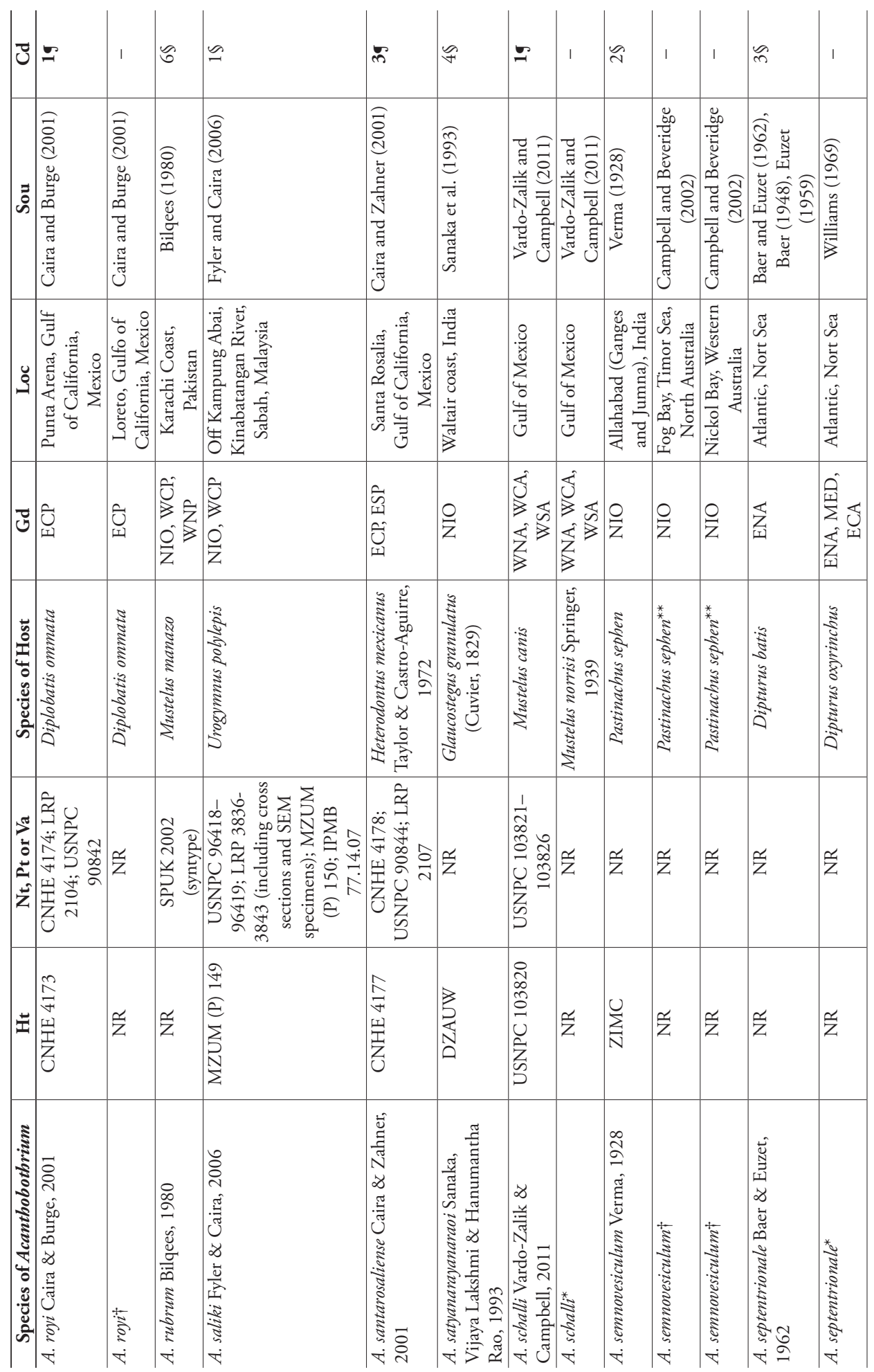




\begin{tabular}{|c|c|c|c|c|c|c|c|c|c|c|c|}
\hline J & 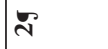 & サ & 1 & S & $\approx$ & 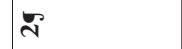 & $\Xi$ & 1 & 1 & 1 & $\approx$ \\
\hline$\ddot{\circ}$ & 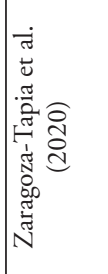 & 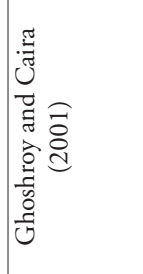 & 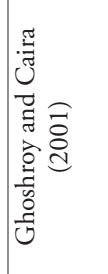 & 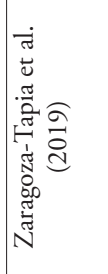 & 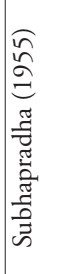 & 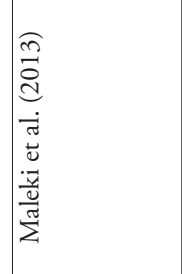 & 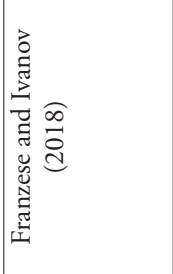 & 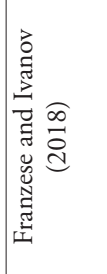 & 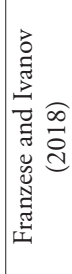 & 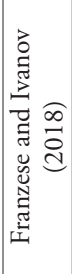 & 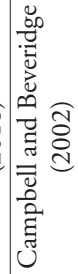 \\
\hline : & 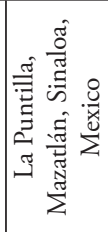 & 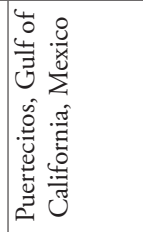 & 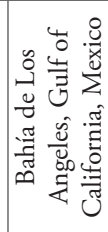 & 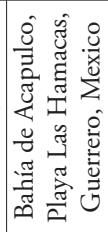 & 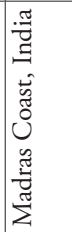 & 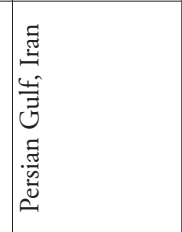 & 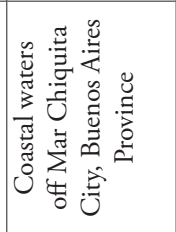 & 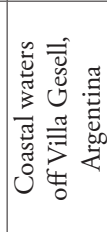 & 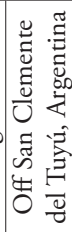 & 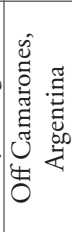 & 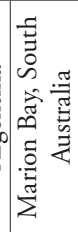 \\
\hline 고 & 式 & 它 & 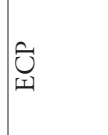 & 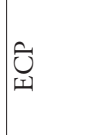 & $\hat{z}$ & $\frac{0}{z}$ & 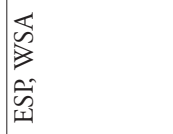 & 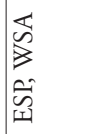 & 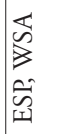 & 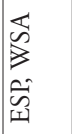 & $\begin{array}{l}\tilde{n} \\
\overrightarrow{3}\end{array}$ \\
\hline 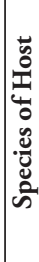 & \begin{tabular}{|l}
$\sqrt{2}$ \\
$\vdots$ \\
3 \\
3 \\
3 \\
3 \\
3 \\
3 \\
3
\end{tabular} & 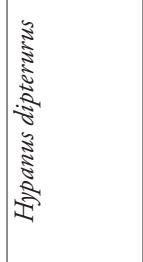 & 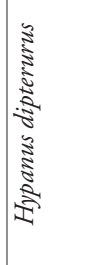 & 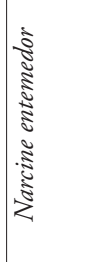 & 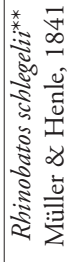 & 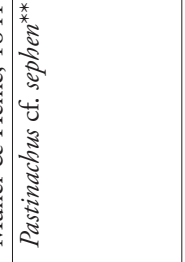 & 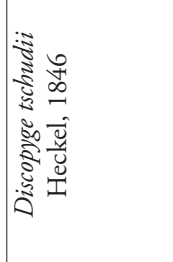 & 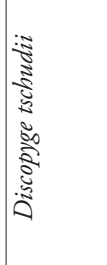 & 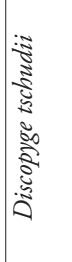 & 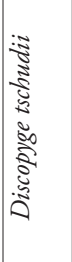 & 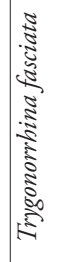 \\
\hline 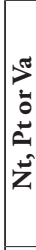 & 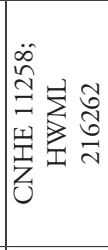 & 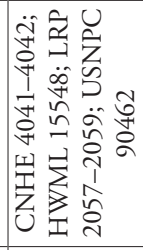 & 号 & 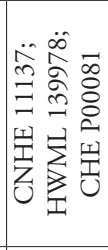 & 号 & 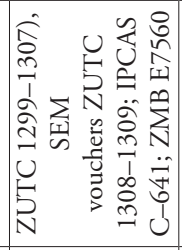 & 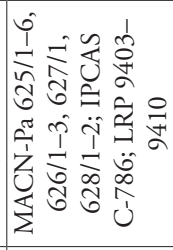 & 艺 & 艺 & 点 & 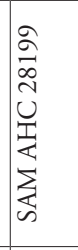 \\
\hline ث & 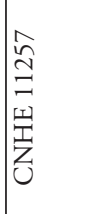 & 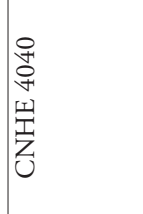 & $\frac{\tilde{z}}{z}$ & 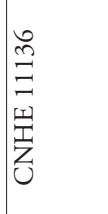 & 光 & 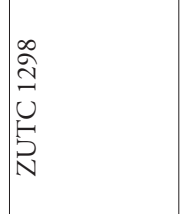 & 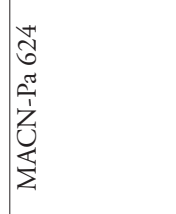 & 孚 & 孚 & 孚 & 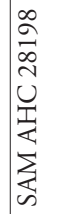 \\
\hline 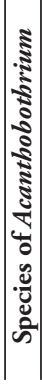 & 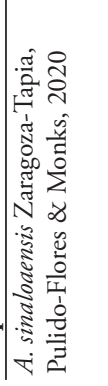 & 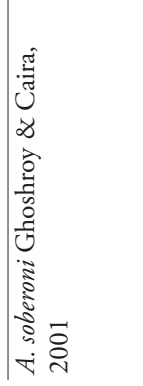 & 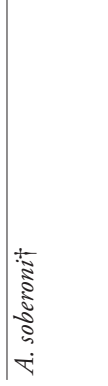 & 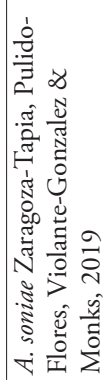 & 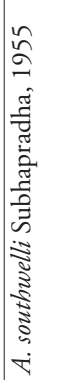 & 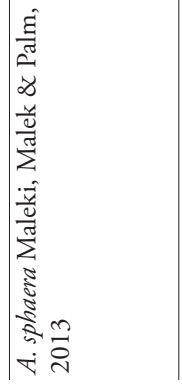 & 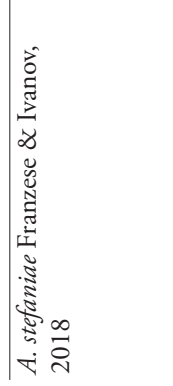 & 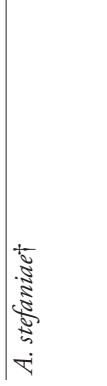 & 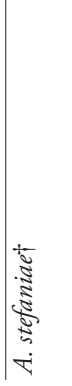 & 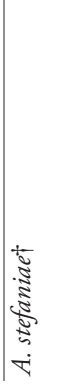 & 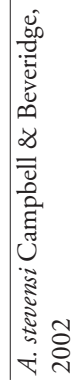 \\
\hline
\end{tabular}




\begin{tabular}{|c|c|c|c|c|c|c|c|c|c|c|c|c|c|}
\hline U & 1 & 1 & 艺 & I & 芰 & $\overline{4}$ & $\approx$ & 1 & 满 & 1 & 1 & $F$ & $\stackrel{\sim}{\sim}$ \\
\hline$\ddot{\circ}$ & 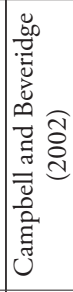 & 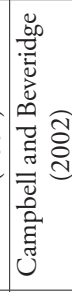 & $\mid \begin{array}{c}\mathbb{E} \\
\hat{2} \\
己 \\
0 \\
0 \\
0 \\
0 \\
0 \\
0\end{array}$ & 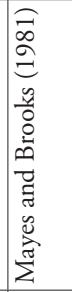 & 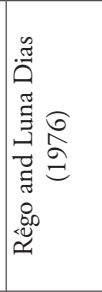 & 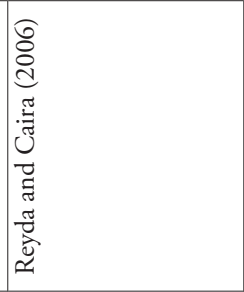 & 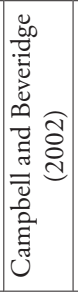 & 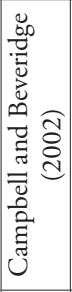 & 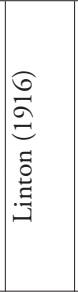 & 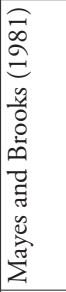 & 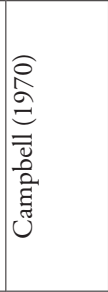 & 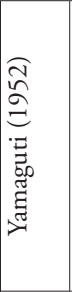 & 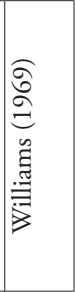 \\
\hline : & 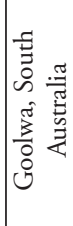 & 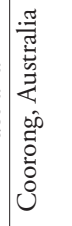 & 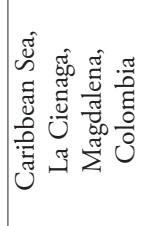 & 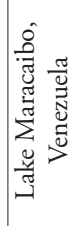 & 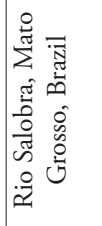 & 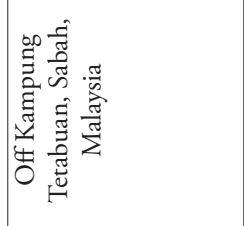 & 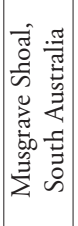 & 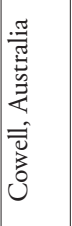 & 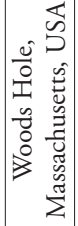 & 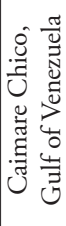 & 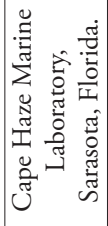 & 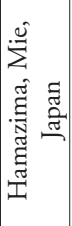 & 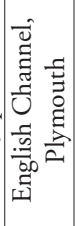 \\
\hline సే & $\hat{\tilde{z}}$ & 芦 & 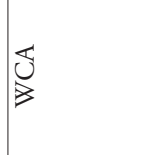 & 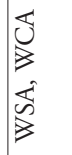 & 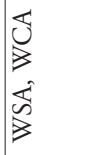 & $\begin{array}{l}\hat{U} \\
\vdots \\
0 \\
\dot{z}\end{array}$ & $\underset{|c|}{0}$ & $\underset{\sim}{\stackrel{0}{\mid c}}$ & 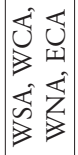 & 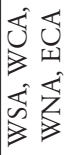 & 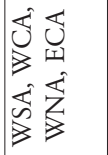 & $\mid \vec{z}$ & 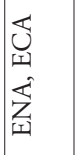 \\
\hline 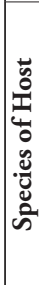 & 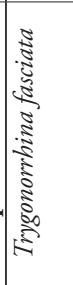 & 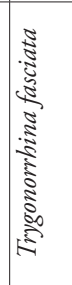 & 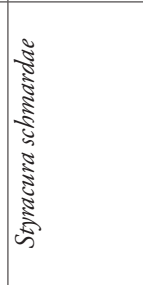 & 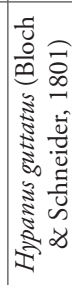 & 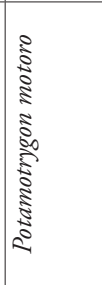 & 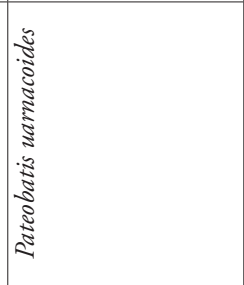 & 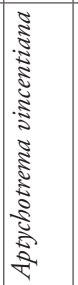 & 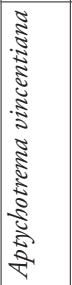 & 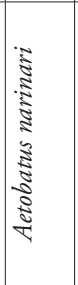 & 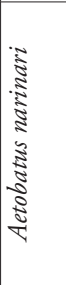 & 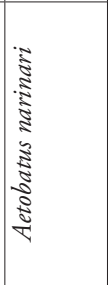 & 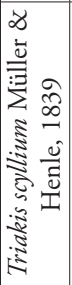 & 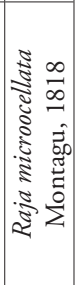 \\
\hline 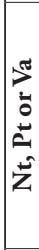 & $\frac{r}{z}$ & $\frac{\kappa}{z}$ & 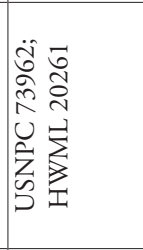 & $\frac{\widetilde{z}}{z}$ & 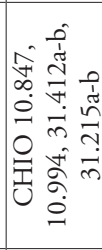 & 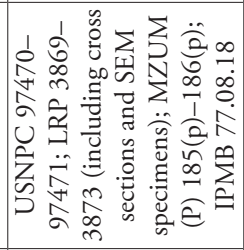 & 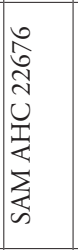 & 学 & 号 & 孚 & 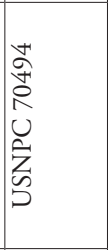 & 孚 & 孚 \\
\hline 考 & 兄 & $\frac{a}{z}$ & $\begin{array}{l}\vec{\Xi} \\
\propto \\
\hat{n} \\
0 \\
\tilde{z} \\
\tilde{\omega}\end{array}$ & 学 & 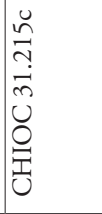 & 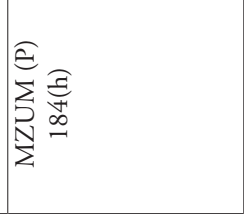 & 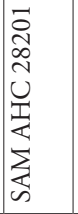 & 孚 & 孚 & 孚 & 号 & 孚 & 吕 \\
\hline 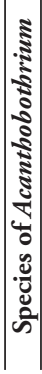 & 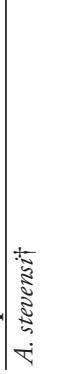 & 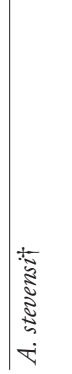 & 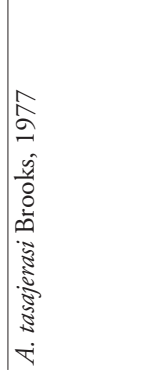 & 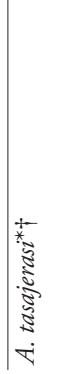 & 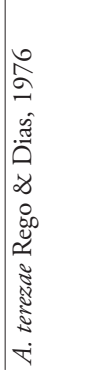 & 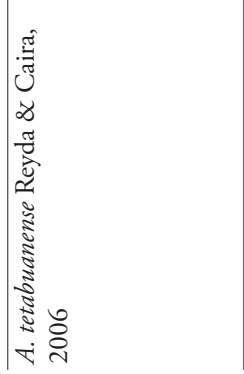 & 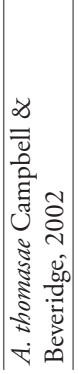 & 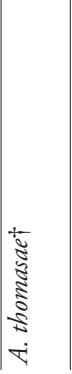 & 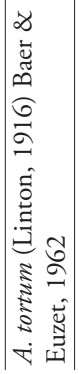 & $\left.\right|_{+} ^{+}$ & 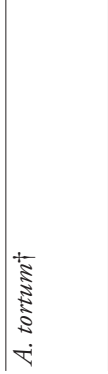 & 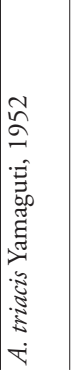 & 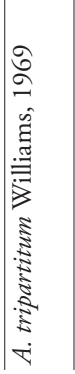 \\
\hline
\end{tabular}




\begin{tabular}{|c|c|c|c|c|c|c|c|c|c|c|c|c|c|}
\hline J & 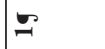 & $\frac{++}{d}$ & S. & $\cong$ & I & $\stackrel{+}{\sim}$ & 1 & 1 & $\stackrel{+}{\sim}$ & (5) & $\check{\sim}$ & $\ddot{m}$ & $\mathscr{\sigma}$ \\
\hline$\ddot{b}$ & 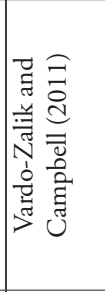 & 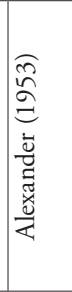 & 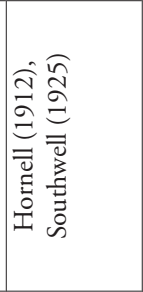 & 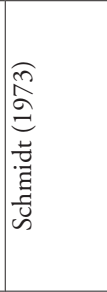 & 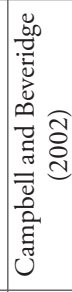 & 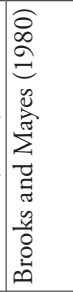 & 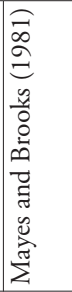 & 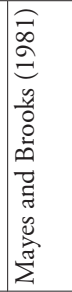 & 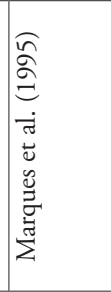 & 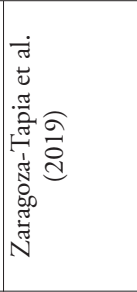 & 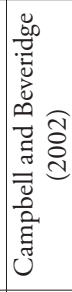 & 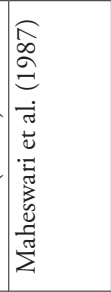 & 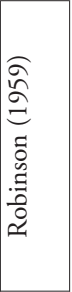 \\
\hline : & 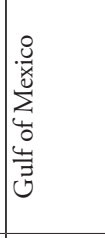 & 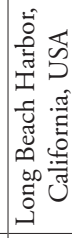 & 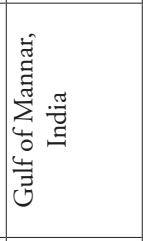 & 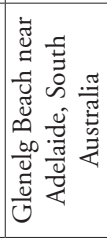 & 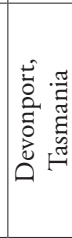 & 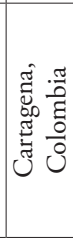 & 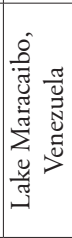 & 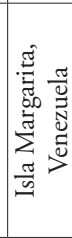 & 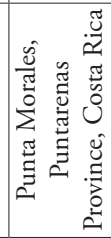 & 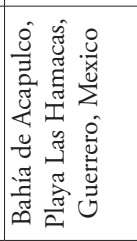 & 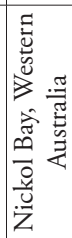 & 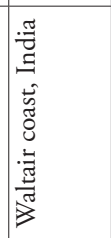 & 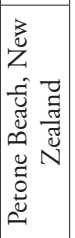 \\
\hline స્త & 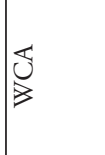 & 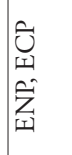 & 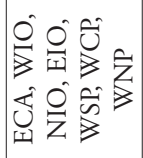 & $\hat{\bar{s}}$ & $\begin{array}{l}\hat{\omega} \\
0 \\
0 \\
0 \\
0\end{array}$ & ठ্ & 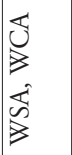 & 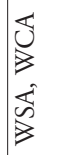 & 式 & 式 & $\frac{O}{z}$ & $\begin{array}{l}0 \\
0 \\
Z \\
0 \\
0 \\
0 \\
0 \\
3\end{array}$ & $\tilde{\tilde{z}}$ \\
\hline 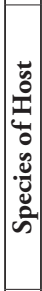 & 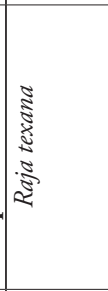 & 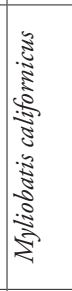 & 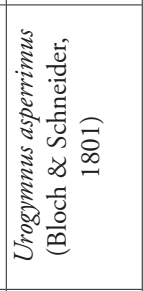 & 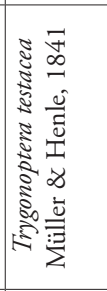 & 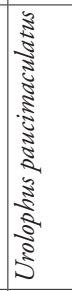 & 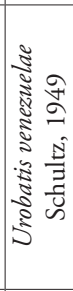 & 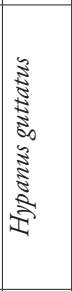 & 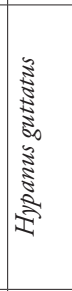 & 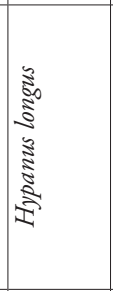 & 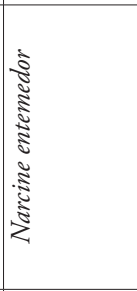 & 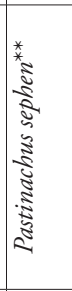 & 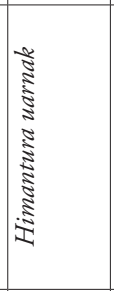 & 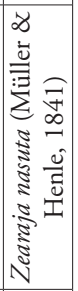 \\
\hline 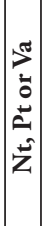 & 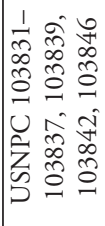 & 号 & 号 & 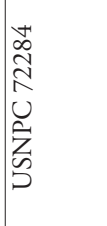 & $\frac{r}{z}$ & 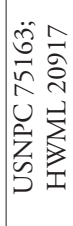 & 号 & $\tilde{z}$ & 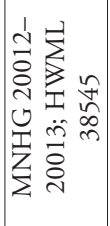 & 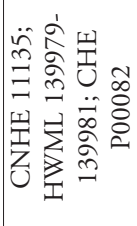 & 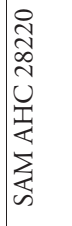 & 号 & 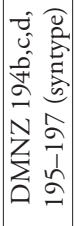 \\
\hline $\mathbf{\pm}$ & 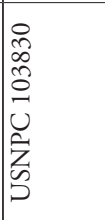 & 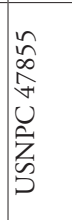 & 吕 & 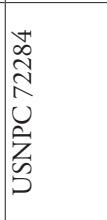 & 号 & 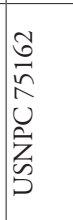 & 号 & 号 & 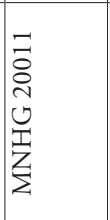 & 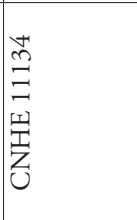 & 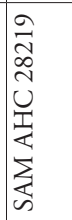 & 号 & 吕 \\
\hline 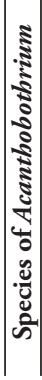 & 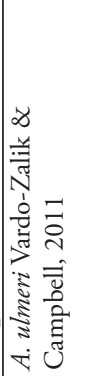 & 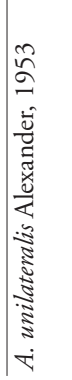 & 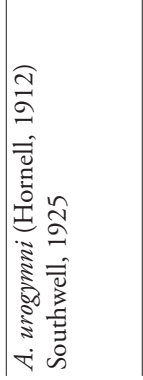 & 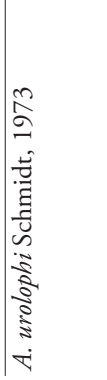 & 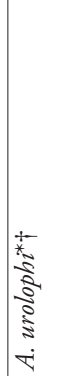 & 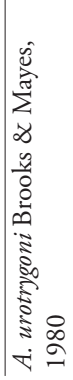 & 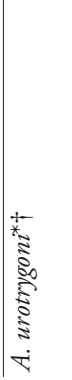 & 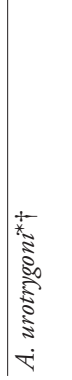 & 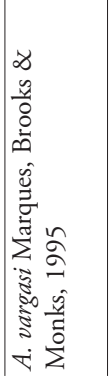 & 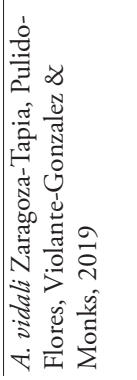 & 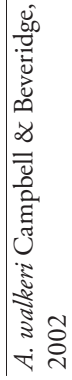 & 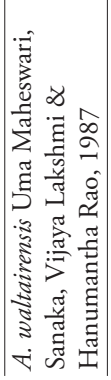 & 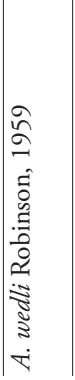 \\
\hline
\end{tabular}




\begin{tabular}{|c|c|c|c|c|c|c|c|c|c|c|}
\hline & & $=$ & 莣 & & in & $\cong$ & 䒚 & ' & 1 & \\
\hline . & 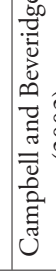 & 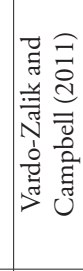 & 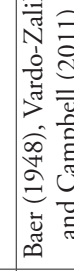 & & 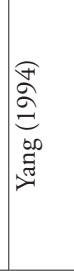 & 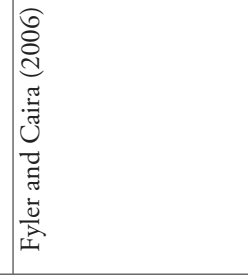 & 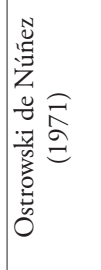 & 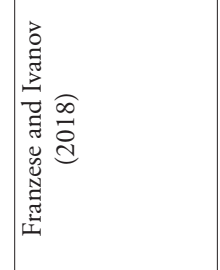 & 焉 & 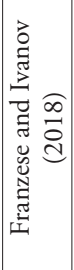 \\
\hline 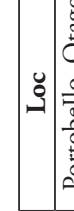 & . & 聯 & 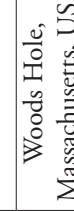 & & 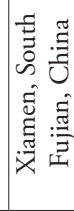 & 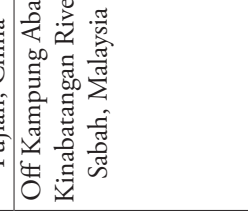 & 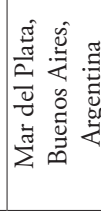 & 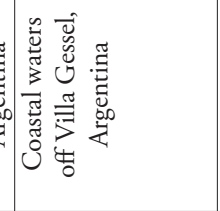 & 童 & 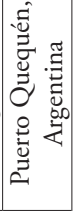 \\
\hline త⿹丁口⿹ & $\hat{\tilde{z}}$ & $\mathbb{B}$ & 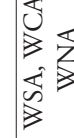 & & \begin{tabular}{|l}
0 \\
$Z$ \\
0 \\
0 \\
\end{tabular} & $\begin{array}{l}\tilde{\tilde{z}} \\
\hat{\beta} \\
\hat{O} \\
\dot{z}\end{array}$ & 质 & $\begin{array}{l}\overleftarrow{b} \\
\ddot{z}\end{array}$ & 萠 & 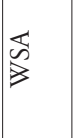 \\
\hline $\begin{array}{l}0 \\
0 \\
0\end{array}$ & 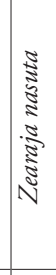 & 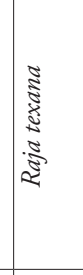 & 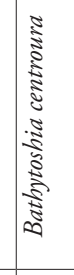 & 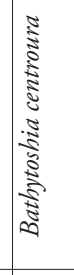 & 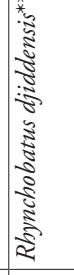 & 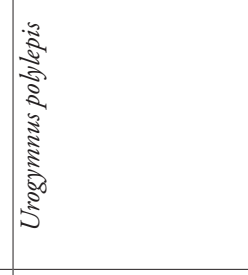 & 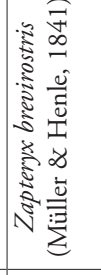 & 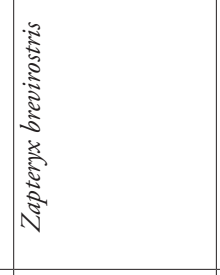 & 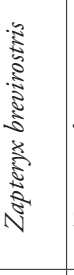 & 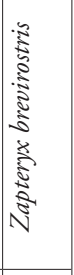 \\
\hline 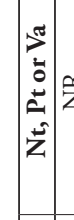 & $\tilde{z}$ & 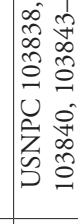 & 40 & $\frac{1}{z}$ & $\tilde{z}$ & 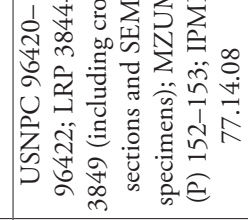 & & 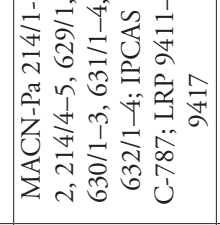 & & 辕 \\
\hline \pm & $\tilde{z}$ & 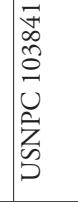 & $\tilde{z}$ & $\tilde{z}$ & $\tilde{z}$ & $\begin{array}{l}\overrightarrow{5} \\
\qquad \\
\bar{E} \\
\vdots \\
\frac{N}{2}\end{array}$ & 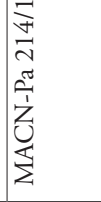 & $\tilde{z}$ & 苂 & z̃ \\
\hline 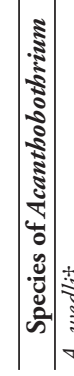 & |ृं & 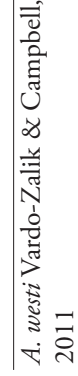 & 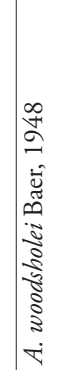 & 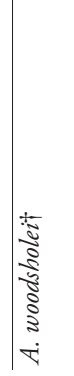 & 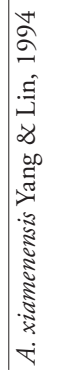 & 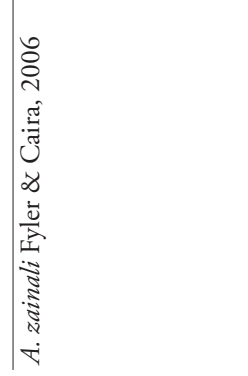 & 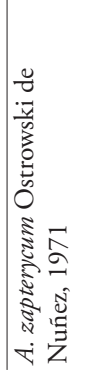 & 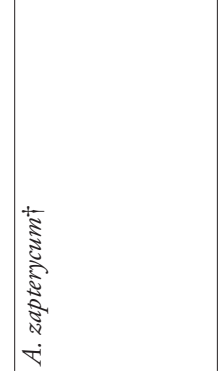 & 部 & 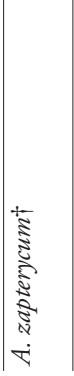 \\
\hline
\end{tabular}




\begin{tabular}{|c|c|c|c|c|c|c|}
\hline $\bar{U}$ & I & $\Xi$ & $\mathscr{\sigma}$ & 1 & 1 & 1 \\
\hline & 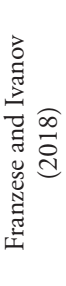 & 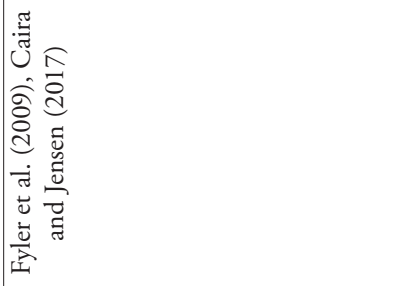 & 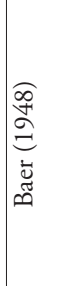 & 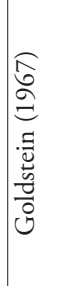 & 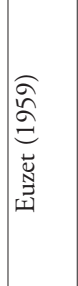 & 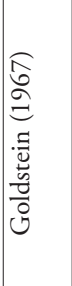 \\
\hline 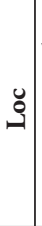 & 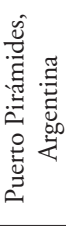 & 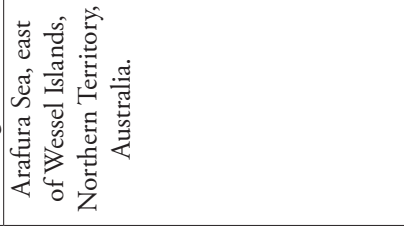 & 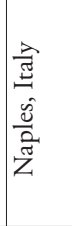 & 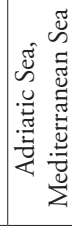 & 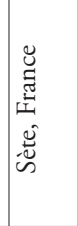 & 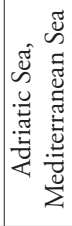 \\
\hline$\vec{J}$ & 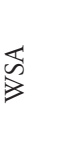 & $\begin{array}{l}\tilde{U} \\
\hat{w} \\
\hat{\tilde{n}} \\
\hat{z}\end{array}$ & n. & 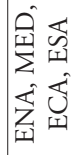 & 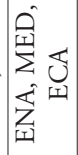 & 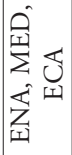 \\
\hline 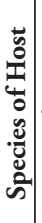 & 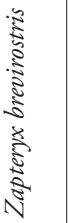 & 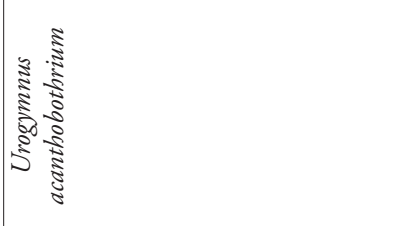 & 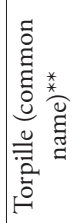 & 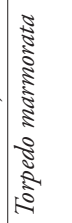 & 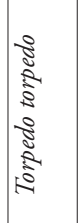 & 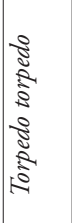 \\
\hline 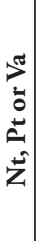 & 㟔 & 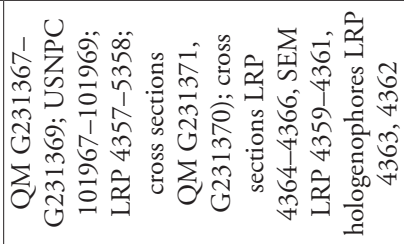 & $\tilde{z}$ & 觉 & $\tilde{z}$ & 咅 \\
\hline $\mathbf{\pm}$ & 孚 & 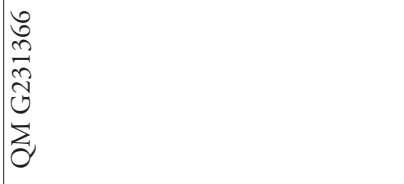 & 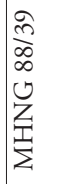 & 觉 & $\tilde{z}$ & $\tilde{z}$ \\
\hline 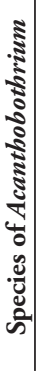 & 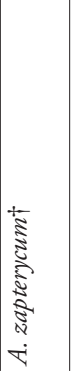 & 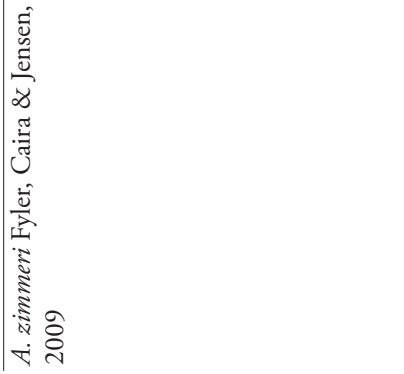 & 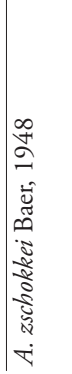 & 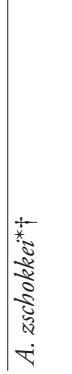 & 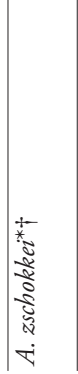 & 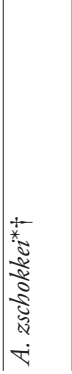 \\
\hline
\end{tabular}




\section{Results}

The information obtained from the metadata analysis (Table 1) is comprised of 336 reports of the 201 valid species of Acanthobothrium. The list includes the type host of each species, type locality, and additional hosts and/or localities. Five of the elasmobranchs that were reported as hosts of Acanthobothrium were only identified to genus and four others are reported as "cf." (= similar to) (see Table 1).

The type localities where species of Acanthobothrium have been reported is shown in Figure 1. The currently known diversity of sharks comprises 517 species (34 families); of these, 19 species of sharks (eight families) have been reported to be parasitized by species of Acanthobothrium (Fig. 2). Eighteen of the 201 valid species have been described from sharks. The families of sharks that have the highest number of reports are Orectolobidae (three different species of Acanthobothrium), Heterodontidae (five species) and Triakidae (six species) (Fig. 2B). In contrast, currently known diversity of rays comprises 637 species ( 26 families); of these, 95 species (18 families) have been reported to be parasitized by species of Acanthobothrium (Fig. 3). Of the 201 valid species of Acanthobothrium, 182 have been described from rays. The families of rays that have the highest number of reports are Rajidae (20 species of Acanthobothrium) and Dasyatidae (70 species) (Fig. 3B).

Species of Acanthobothrium are not evenly grouped in the different categories. In Category 1 there are 55 species, 44 in Category 2, 19 in Category 3, 37 in Category 4, 17 in Category 5, 14 in Category 6, four in Category 7, four in Category 8, and three in Category 9. Although there is a Category 10, species in that category also are in grouped with those in Category 8 because their characteristics are thought to fall into both categories (Table 1). The categories of four species of Acanthobothrium were classified as unknown ("?") because the original descriptions do not have sufficient information for assignment in one of the ten categories (Table 1).

\section{Discussion}

Currently, 517 species of sharks have been described worldwide with $3.7 \%$ (19 of the 517 species) have been reported as hosts for species of Acanthobothrium (Fig. 2C). In contrast, 637 species of rays have been described with 14.9\% (95 of the 637 species) have been reported as hosts for species of Acanthobothrium (Fig. 3C). Estimates of cestode diversity in elasmobranchs discussed by Caira (2011) assumes that the fauna of cestodes of a species of elasmobranchs does not vary substantially across in its distribution. Knowledge of life cycles are essential in understanding the distribution of species of Acanthobothrium; however, for this study it is assumed that the distribution of adults of these parasites normally is limited to that of its normal definitive host. Thus, it is hypothesized that the limits of the distribution of the host limits the species of its parasites to the same biogeographic regions proposed for the distribution of elasmobranchs by Last et al. (2016b). It is recognized that an infected elasmobranch 
A

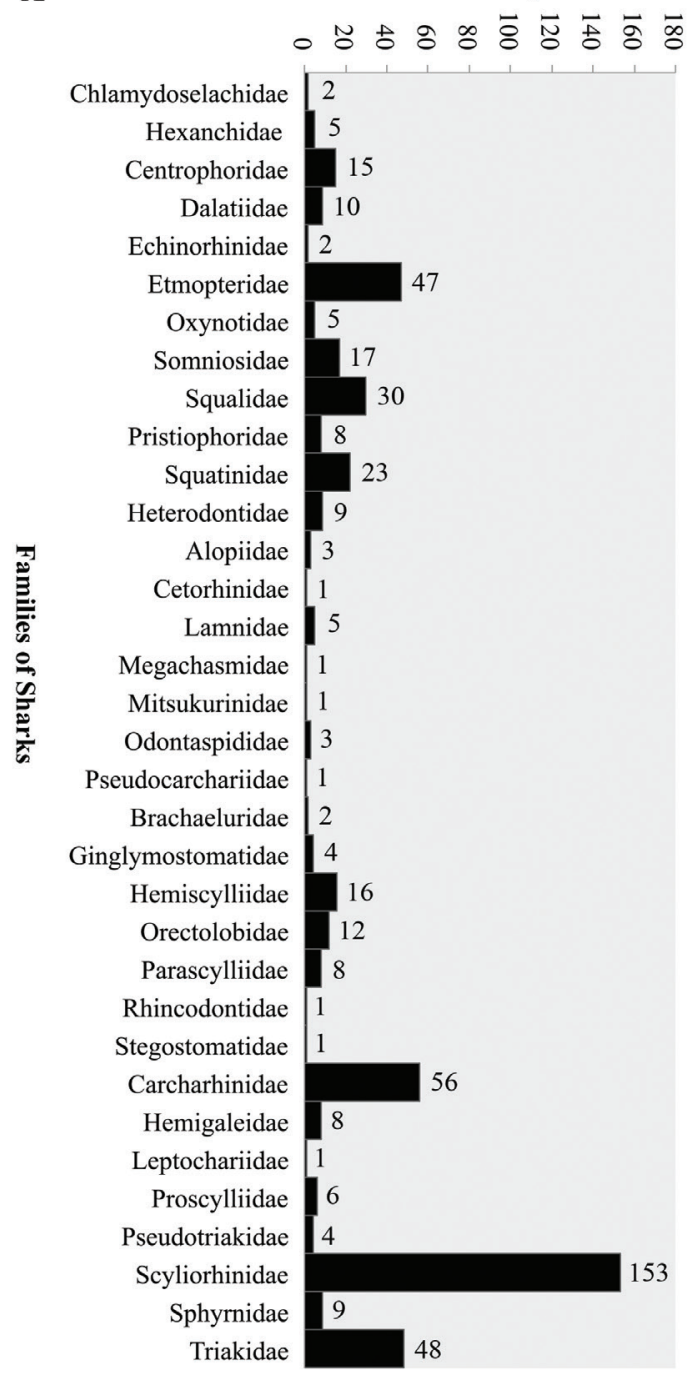

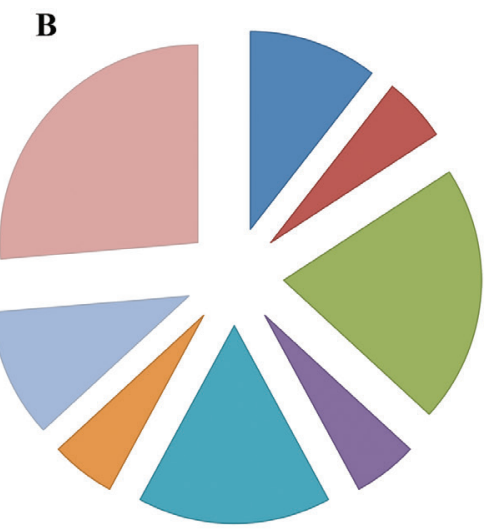

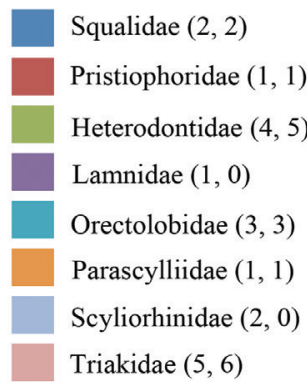

C

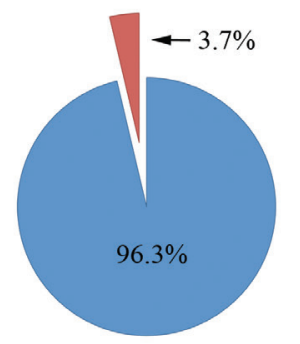

Figure 2. Families of sharks: A number of species of sharks per family B number of species of sharks parasitized by species of Acanthobothrium. Note: The first number within parentheses corresponds to the number of species of shark that have been reported as hosts of Acanthobothrium and the second is the number of species that have been described from that Family $\mathbf{C}$ percentage of species of shark reported to be parasitized within the total number of families of sharks- note: Red color = parasitized; Blue color = not parasitized.

could move outside of the region where it has been designated, but until an extension to its distribution has been reported, it must be assumed that the normal distribution for each species of parasite also is that same designated region. The information in the table will be subject to future research, not forgetting that there is a lack of knowledge of the life cycle of the species of Acanthobothrium; a partial life cycle of a single species 
A

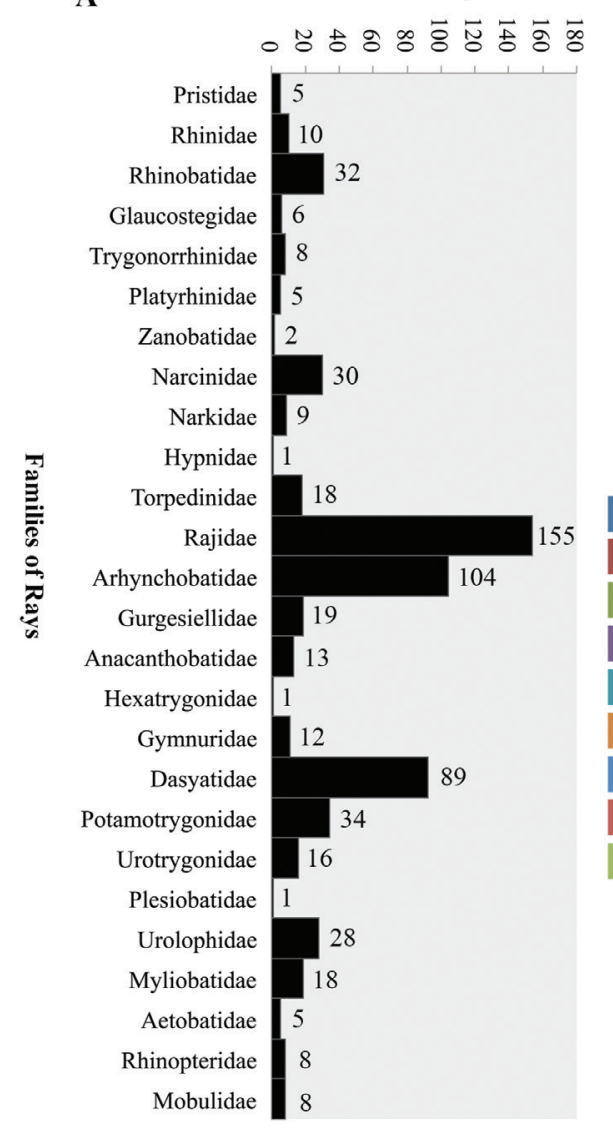

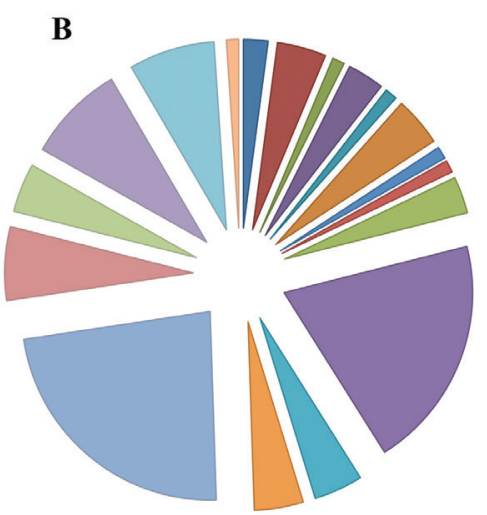

Rhinidae $(2,14)$

Rhinobatidae $(4,5)$ Glaucostegidae $(1,1)$ Trygonorrhinidae $(3,7)$ Platyrhinidae $(1,1)$ Narcinidae $(4,11)$ Narkidae $(1,1)$ Hypnidae $(1,1)$ Torpedinidae $(3,3)$

Rajidae $(19,20)$
Arhynchobatidae $(4,3)$
Gymnuridae $(4,9)$
Dasyatidae $(22,70)$
Potamotrygonidae $(6,8)$
Urotrygonidae $(4,5)$
Urolophidae $(8,4)$
Myliobatidae $(7,11)$
Aetobatidae $(1,8)$

C

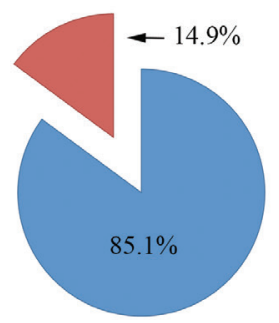

Figure 3. Families of rays: $\mathbf{A}$ number of species of rays per family $\mathbf{B}$ number of species of rays parasitized by species of Acanthobothrium. Note: The first number within parentheses corresponds to the number of species of ray that have been reported as hosts of Acanthobothrium and the second is the number of species that have been described from that Family $\mathbf{C}$ percentage of species of rays reported to be parasitized within the total number of families of rays- note: Red color = parasitized; Blue color = not parasitized.

has been reported (Holland and Wilson 2009). Publication of molecular sequences for more species will provide new discoveries in this subject.

The information in the Figures 1 and 4 indicates that there is an absence of reports from several regions of the world, such as ECA, ESA, WIO, ARC, and SOC. According to the percentages of species of elasmobranchs that have been reported as hosts of species of Acanthobothrium, we can infer that there are still many new species of Acanthobothrium to be discovered. In the GenBank database records, molecular sequences 


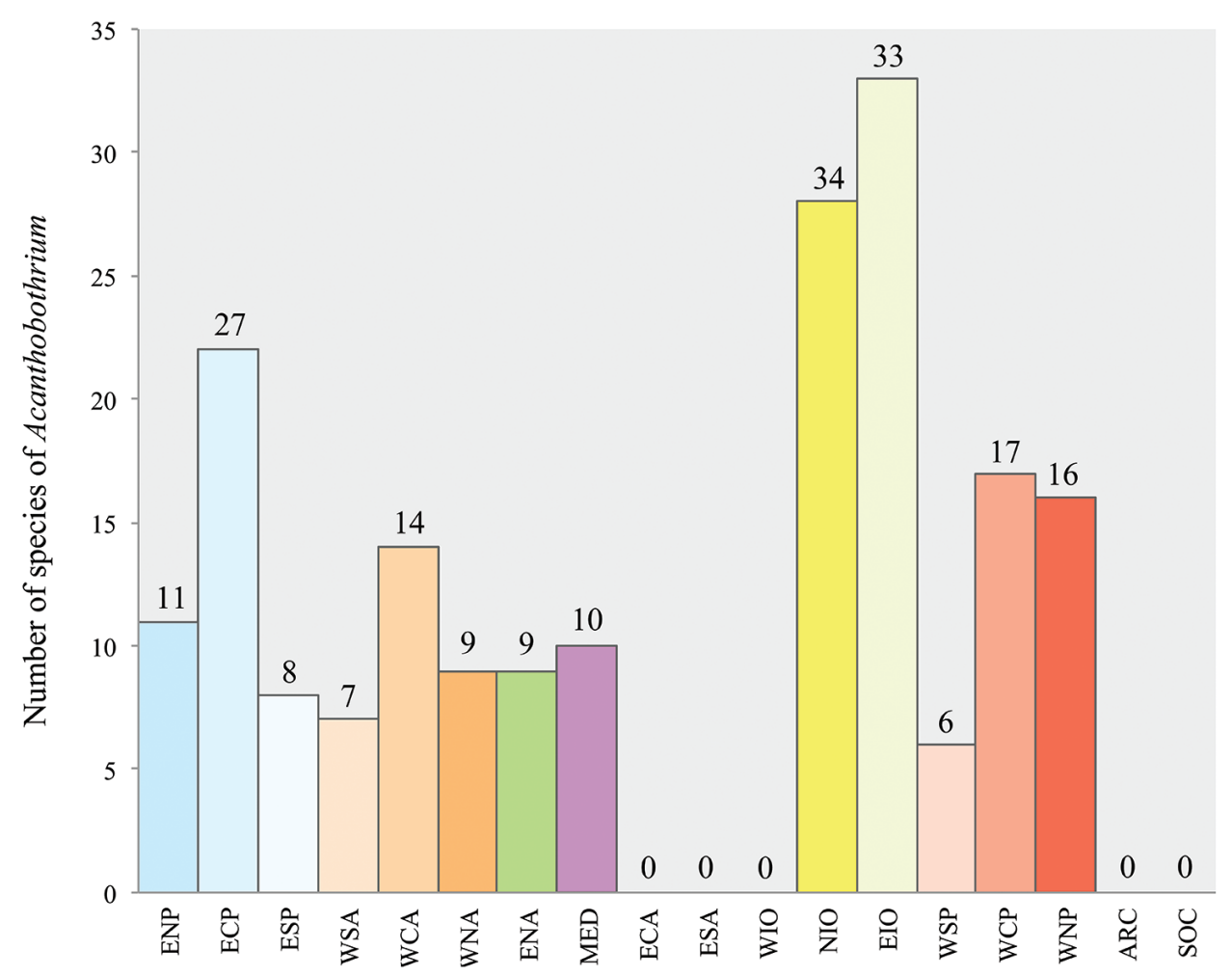

Biogeographic regions

Figure 4. Number of species of Acanthobothrium reported from elasmobranchs in each biogeographic region (Last et al. 2016b).

of only 16 of the 201 species of Acanthobothrium have been reported. However, more molecular information about species of Acanthobothrium is required for future analyzes, both for identification and life cycle studies; these would provide more solid information for delimiting distributions.

In Table 1, Acanthobothrium chilensis Rêgo, Vicente \& Herrera, 1968, was included for reference, although it was described from a fish, Sarda chiliensis (Cuvier, 1832) (Perciformes: Scombridae) (see Rêgo et al. 1968). Extensive recent studies of this species of fish (Chero et al. 2016; Luque et al. 2016) failed to report $A$. chilensis; there is only the report by Rêgo et al. (1968). The report of the host for this species of Acanthobothrium likely is an accidental infection and not a normal host.

According to Fyler et al. (2009) and Franzese and Ivanov (2018), species of Acanthobothrium appear to exhibit oioxenous specificity for their elasmobranch hosts. In the present metadata analysis, for species exclusively in elasmobranchs, $83 \%$ of the species of Acanthobothrium show remarkable host specificity for their definitive host, and thus, should be considered to be an oioxenous species. In contrast, 34 of the 200 
species (17\%) of Acanthobothrium have been reported in more than one species of elasmobranch (Table 1). However, with the metadata analysis of the distribution of the hosts and the reports of the species of Acanthobothrium, 45 of the type specimens of Acanthobothrium require confirmation of the host (Table 1) because some appear to be problematic identifications and other hosts were reported as "cf." or only as an unidentified member of a particular genus In addition, there are reports of species of Acanthobothrium that suggest misidentification of the parasites; these should reevaluated in future studies. To mention obvious cases, A. batailloni has been reported from the Mediterranean Sea and from the Pacific coast of Peru and Chile and A. brevissime has been reported from the Gulf of Mexico and the Pacific coast of Peru.

The categorical method developed by Ghoshroy and Caira (2001) was proposed in order to delimit the number of taxonomic comparisons when describing new species. Using the method of Ghoshroy and Caira (2001), which focused only on species from the Americas, Fyler and Caira (2006) later applied the same methodology to biodiversity data for species from other regions; those works are augmented by this study. Of the 201 known species of Acanthobothrium, 13 have been classified in more than one category (see category designations in Table 1) because some characteristics of those species overlap with those of more than one category (see descriptions found in Zschokke 1888; Linton 1890; Baer 1948; Alexander 1953; Euzet 1955; Riser 1955; Yamaguti 1959; Goldstein 1964; Williams 1969; Goldstein et al. 1969; Appy and Dailey 1973; Severino and Sarmiento 1979; Marques et al. 1997; Reyda 2008). This does not decrease the usefulness of the categorical method as a tool for the initial stages in identification.

Having more information, such as molecular sequences, could solve some problems in identification, such as the two cases mentioned above. A species of Acanthobothrium that has been assigned to more than one category suggests that the categories still need some refining, or it is an example of cryptic species that cannot be distinguished without molecular information. However, molecular information cannot replace morphological descriptions of species. One reason is the lack of material for sequencing of the vast majority of already-known species. Morphology also augments molecular data in studies of the phylogeny of platyhelminths (Zamparo et al. 2001; Littlewood 2008). A complete phylogenetic hypothesis based on total evidence (morphological and molecular data) such as that of Littlewood (2008) for any major group of cestodes is still distant. Until that time, a categorical method provides the easiest and most direct method for selection of a group of species similar to a new species of Acanthobothrium. This updated database includes the category designation for each species described to date will be an important tool for the future taxonomic studies.

\section{Acknowledgements}

The authors would like to thank to Luis García-Prieto (CNHE) for providing important bibliographic references and the Consejo Nacional de Ciencia y Tecnología (CONACYT) for a doctoral scholarship (no. 432427) to FZ-T. 


\section{References}

Alexander CG (1953) Five new species of Acanthobothrium (Cestoda: Tetraphyllidea) from southern California rays. Journal of Parasitology 39: 481-486. https://doi.org/10.2307/3273847

Alves PV, de Chambrier A, Scholz T, Luque JL (2017) Annotated checklist of fish cestodes from South America. ZooKeys 650: 1-205. https://doi.org/10.3897/zookeys.650.10982

Amaral CRL, Pereira F, Silva DA, Amorim A, de Carvalho EF (2018) The mitogenomic phylogeny of the Elasmobranchii (Chondrichthyes). Mitochondrial DNA A DNA Mapp Seq Anal 29: 867-878. https://doi.org/10.1080/24701394.2017.1376052

Appy RG, Dailey MD (1973) Two new species of Acanthobothrium (Cestoda: Tetraphyllidea) from elasmobranchs of the eastern Pacific. Journal of Parasitology 59: 817-820. https:// doi.org/10.2307/3278414

Baer JG (1948) Contributions a l'étude des cestodes de sélaciens I-IV. Bulletin de la Société des Sciences Naturelles de Neuchâtel 71: 63-122.

Baer JG, Euzet L (1962) Revision critique des Cestodes Tétraphyllides décrits par T. Southwell ( $1^{\text {ere }}$ Partie). Extrait du Bulletin de la Société neuchâteloise des Sciences naturelles 85: $143-172$.

Bilqees FM (1980) Three new species of Acanthobothrium Van Beneden (Cestoda: Tetraphyllidea: Onchobothriidae) in Myrmillo manazo (blk.) of Karachi coast. Pakistan Journal of Zoology 12: 239-246.

Brooks DR (1977) Six new species of tetraphyllidean cestodes, including a new genus, from a marine stingray Himantura schmardae (Werner, 1904) from Colombia. Proceedings of the Helminthological Society of Washington 44: 51-59.

Brooks DR, Mayes MA (1978) Acanthobothrium electricolum sp. n. and A. lintoni Goldstein, Henson, and Schlicht, 1969 (Cestoda: Tetraphyllidea) from Narcine brasiliensis (Olfers) (Chondrichthyes: Torpedinidae) in Colombia. Journal of Parasitology 64: 617-619. https://doi.org/10.2307/3279945

Brooks DR, Mayes MA (1980) Cestodes in four species of euryhaline stingrays from Colombia. Proceedings of the Helminthological Society of Washington 47: 22-29.

Brooks DR, Mayes MA, Thorson TB (1981) Systematic review of cestodes infecting freshwater stingrays (Chondrichthyes: Potamotrygonidae) including four new species from Venezuela. Proceedings of the Helminthological Society of Washington 48: 43-64.

Brooks DR, McCorquodale S (1995) Acanthobothrium nicoyaense n. sp. (Eucestoda: Tetraphyllidea: Onchobothriidae) in Aetobatus narinari (Euphrasen) (Chondrichthyes: Myliobatiformes: Myliobatidae) from the Gulf of Nicoya, Costa Rica. Journal of Parasitology 81: 244-246. https://doi.org/10.2307/3283927

Caira JN (2011) Synergy advances parasite taxonomy and systematics: an example from elasmobranch tapeworms. Parasitology 138: 1675-1687. https://doi.org/10.1017/ S0031182011000643

Caira JN, Burge AN (2001) Three new species of Acanthobothrium (Cestoda: Tetraphyllidea) from the ocellated electric ray, Diplobatis ommata, in the Gulf of California, Mexico. Comparative Parasitology 68: 52-65.

Caira JN, Jensen K, Barbeau E (2019) Global Cestode Database. http://tapewormdb.uconn.edu/ 
Caira JN, Jensen K, Ivanov VA (2017) Onchoproteocephalidea II. In: Caira JN, Jensen K (Eds) Planetary biodiversity inventory (2008-2017): tapeworms from vertebrate bowels of the earth Special Publication, Natural History Museum, The University of Kansas Lawrence, Kansas, 290-315.

Caira JN, Zahner SD (2001) Two new species of Acanthobothrium Beneden, 1849 (Tetraphyllidea: Onchobothriidae) from horn sharks in the Gulf of California, Mexico. Systematic Parasitology 50: 219-229. https://doi.org/10.1023/A:1012241913722

Campbell RA (1969) New species of Acanthobothrium (Cestoda: Tetraphyllidea) from Chesapeake Bay, Virginia. Journal of Parasitology 55:559-570. https://doi.org/10.2307/3277298

Campbell RA (1970) Notes on tetraphyllidean cestodes from the Atlantic Coast of North America, with descriptions of two new species. Journal of Parasitology 56: 498-508. https://doi.org/10.2307/3277613

Campbell RA, Beveridge I (2002) The genus Acanthobothrium (Cestoda: Tetraphyllidea: Onchobothriidae) parastic in Australian elasmobranch fishes. Invertebrate Systematics 16: 273-344. https://doi.org/10.1071/IT01004

Carvajal-G. J, Goldstein RJ (1969) Acanthobothrium psammobati n. sp. (Cestoda: Tetraphyllidea: Onchobothriidae) from the skate, Psammobatis scobina (Chondrichthyes: Rajidae) from Chile. Zoologischer Anzeiger 182: 432-435.

Carvajal-G. J, Goldstein RJ (1971) Acanthobothrium annapinkiensis n. sp. (Cestoda: Tetraphyllidea: Onchobothriidae) from the skate, Raja chilensis (Chondrichthyes: Rajidae) from Chile. Zoologischer Anzeiger 186: 158-162.

Carvajal-G. J, Jeges-G. J (1980) Cestodos parásitos de Myliobatis chilensis Phillipi (Pisces: Myliobatidae), con la descripción de una nueva especie de Acanthobothrium. Anales del Centro de Ciencias del Mar y Limnología 7: 51-56.

Chero J, Sáez G, Iannacone J, Cruces C, Alvariño L, Luque J (2016) Ecología comunitaria de metazoos parásitos del bonito Sarda chiliensis Cuvier, 1832 (Perciformes: Scombridae) de la Costa Peruana. Revista de Investigaciones Veterinarias del Perú 27: 539-555. https://doi. org/10.15381/rivep.v27i3.12008

Concha FJ, Caira JN, Ebert DA, Pompert JHW (2019) Redescription and taxonomic status of Dipturus chilensis (Guichenot, 1848), and description of Dipturus lamillai sp. nov. (Rajiformes: Rajidae), a new species of long-snout skate from the Falkland Islands 4590: 501-524. https://doi.org/10.11646/zootaxa.4590.5.1

Cornford EM (1974) Two tetraphyllidean cestodes from Hawaiian stingrays. Journal of Parasitology 60: 942-948. https://doi.org/10.2307/3278520

Dailey MD, Mudry DR (1968) Two new species of cestodes from California rays. Journal of Parasitology 54: 1141-1143. https://doi.org/10.2307/3276979

Del Moral-Flores LF, Morrone JJ, Alcocer-Durand J, Espinosa-Pérez H, Pérez-Ponce de León G (2015) Lista patrón de los tiburones, rayas y quimeras (Chondrichthyes, Elasmobranchii, Holocephali) de México. Arxius de Miscel-lània Zoològica 13: 47-163. https://doi. org/10.32800/amz.2015.13.0047

Dollfus RP (1926) Sur Acanthobothrium crassicolle K. Wedl 1855. Bulletin Société Zool France 51: 464-470. 
Drummond FH (1937) Cestoda. Lady Julia Percy Island. Reports of the expedition of the McCoy Society for Field Investigations and Research. Proceedings of the Royal Society of Victoria 49: 401-404.

Escalante-A. H (1986) Cestodes de elasmobranquios de la costa peruana. Revista de Ciencias Universidad Nacional Mayor de San Marcos 74: 70-74.

Euzet L (1952) Cestodes tétraphyllides de la côte Atlantique du Maroc et de Mauritanie. (Collection resemblée par R. Ph. Dollfus). Comptes Rendus de la Société des Sciences Naturelles de Maroc 5: 91-96.

Euzet L (1955) Quelques cestodes de Myliobatis aquila L. Receuil des Travaux des Laboratoires de Botanique, Géologie et Zoologie de la Faculté des Sciences de l' Université de Montpellier Série Zoologie 1: 18-27.

Euzet L (1959) Recherches sur les Cestodes Tétraphyllides des Sélaciens de côtes de France. Docteur ès Sciences Naturelles, Montpellier, France: University of Montpellier.

Franzese S, Ivanov VA (2018) Hyperapolytic species of Acanthobothrium (Cestoda: Onchoproteocephalidea) from batoids off Argentina. Parasitology International 67: 431443. https://doi.org/10.1016/j.parint.2018.04.001

Froese R, Pauly D (2019) FishBase. Version (08/2019). World Wide Web electronic. http:// www.fishbase.org

Friggens MM, Brown JH (2005) Niche partitioning in the cestode communities of two elasmobranchs. Oikos 108: 76-84. https://doi.org/10.1111/j.0030-1299.2005.13275.x

Fyler CA (2011) An extremely hyperapolytic Acanthobothrium species (Cestoda: Tetraphyllidea) from the Japanese wobbegong, Orectolobus japonicus (Elasmobranchii: Orectolobiformes) in Taiwan. Comparative Parasitology 78: 4-14. https://doi.org/10.1654/4454.1

Fyler CA, Caira JN (2006) Five new species of Acanthobothrium (Tetraphyllidea: Onchobothriidae) from the freshwater stingray Himantura chaophraya (Batoidea: Dasyatidae) in Malaysian Borneo. Journal of Parasitology 92: 105-125. https://doi.org/10.1645/GE-3522.1 Fyler CA, Caira JN (2010) Phylogenetic status of four new species of Acanthobothrium (Cestoda : Tetraphyllidea) parasitic on the wedgefish Rhynchobatus laevis (Elasmobranchii : Rhynchobatidae): implications for interpreting host associations. Invertebrate Systematics 24: 419-433. https://doi.org/10.1071/IS10034

Fyler CA, Caira JN, Jensen K (2009) Five new species of Acanthobothrium (Cestoda: Tetraphyllidea) from an unusual species of Himantura (Rajiformes: Dasyatidae) from northern Australia. Folia Parasitologica 56: 107-128. https://doi.org/10.14411/fp.2009.016

Ghoshroy S, Caira JN (2001) Four new species of Acanthobothrium (Cestoda: Tetraphyllidea) from the whiptail stingray Dasyatis brevis in the Gulf of California, Mexico. Journal of Parasitology 87: 354-372. https://doi.org/10.1645/0022-3395(2001)087[0354:FNSOA $\mathrm{C}] 2.0 . \mathrm{CO} ; 2$

Goldstein RJ (1964) Species of Acanthobothrium (Cestoda: Tetraphyllidea) from the Gulf of Mexico. Journal of Parasitology 50: 656-661. https://doi.org/10.2307/3276123

Goldstein RJ (1967) The genus Acanthobothrium van Benden, 1849 (Cestoda: Tetraphyllidea). Journal of Parasitology 53: 455-483. https://doi.org/10.2307/3276705 
Goldstein RJ, Henson RN, Schlicht FG (1969) Acanthobothrium lintoni sp. n. (Cestoda: Tetraphyllidea) from the electric ray, Narcine brasiliensis (Olfers) in the Gulf of Mexico. Zoologischer Anzeiger 181: 435-438.

Grace MA, Doosey MH, Denton JSS, Naylor GJP, Bart HLJ, Maisey JG (2019) A new Western North Atlantic Ocean kitefin shark (Squaliformes: Dalatiidae) from the Gulf of Mexico. 2019 4619: 109-120. https://doi.org/10.11646/zootaxa.4619.1.4

Hassan S (1983) Acanthobothrium manteri sp. n. a tetraphyllidean cestode (Onchobothriidae) from Dasyatis sephen. Journal of the Egyptian Society of Parasitology 13: 75-80.

Holland ND, Wilson NG (2009) Molecular Identification of Larvae of a Tetraphyllidean Tapeworm (Platyhelminthes: Eucestoda) in a Razor Clam as an Alternative Intermediate Host in the Life Cycle of Acanthobothrium brevissime. Journal of Parasitology 95: 1215-1217. https://doi.org/10.1645/GE-1946.1

Hornell J (1912) XVIII. New cestodes from Indian fishes. Records of the Indian Museum 7: 197-204. [110 plates] https://doi.org/10.5962/bhl.part.28231

Iannacone J, Avila-Peltroche J, Rojas-Perea S, Salas-Sierralta M, Neira-Cruzado K, PalomaresTorres R, Valdivia-Alarcón S, Pacheco-Silva A, Benvenutto-Vargas V, Ferrario-Bazalar V (2011) Dinámica poblacional de los parásitos metazoos del Pez Guitarra del pacífico Rhinobatos planiceps (Batoidea: Rajiformes) de la zona costera marina de Lima, Perú. Neotropical Helminthology 5: 265-278.

Ivanov VA (2005) A new species of Acanthobothrium (Cestoda: Tetraphyllidea: Onchobothriidae) from the ocellate river stingray, Potamotrygon motoro (Chondrichthyes: Potamotrygonidae), in Argentina. Journal of Parasitology 91: 390-396. https://doi.org/10.1645/ GE-354R1

Ivanov VA, Campbell RA (1998) A new species of Acanthobothrium van Beneden, 1849 (Cestoda: Tetraphyllidea) from Rioraja castelnaui (Chondrichthyes: Rajoidei) in coastal waters of Argentina. Systematic Parasitology 40: 203-212. https://doi.org/10.1023/A:1006049404646 Lacerda ACF, Takemoto RM, Pavanelli GC (2008) Digenea, Nematoda, Cestoda, and Acanthocephala, parasites in Potamotrygonidae (Chondrichthyes) from the upper Paraná River floodplain, states of Paraná and Mato Grosso do Sul, Brazil. Check List 4: 115-122. https://doi.org/10.15560/4.2.115

Last PR, Naylor GJP, Manjali-Matsumoto BM (2016a) A revised classification of the family Dasyatidae (Chondrichthyes: Myliobatiformes) based on new morphological and molecular insights. Zootaxa 4139: 345-368. https://doi.org/10.11646/zootaxa.4139.3.2

Last PR, White WT, de Carvalho MR, Séret B, Stehman MFW, Naylor GJP, McEachran JD (2016b) Rays of the World. Comstock Publishing Associates, a division of Cornell University Press; Clayton South VIC, Australia: CSIRO Publishing, Ithaca, New York, 790 pp.

Léon-Borcéa L (1934) Note préliminaire sur les cestodes de elasmobranchs ou sélaciens de la mer noire. Annales scientifiques de l'Université de Jassy 19: 345-369.

Léon-Borcéa L (1935) Novelle note sur Acanthobothrium ponticum L. Borcéa (n. sp.). Annales scientifiques de l'Université de Jassy 20: 480-481.

Linton E (1890) Notes on Entozoa of marine fishes of New England, with descriptions of several new species. Part II. Annual Report of the Commissioner of Fish and Fisheries for 1887. Washington, D.C., 719-899. https://doi.org/10.5962/bhl.title.995 
Linton E (1908) IX. Helminth fauna of the Dry Tortugas. I. Cestodes. Papers from the Tortugas Laboratory. Carnegie Institucion of Washington, Washington, D.C., 157-190.

Linton E (1916) Notes on two cestodes from the spotted stingray. Journal of Parasitology 3: 34-38. https://doi.org/10.2307/3270745

Littlewood DTJ (2008) Platyhelminth systematics and the emergence of new characters. Parasite 15: 333-341. https://doi.org/10.1051/parasite/2008153333

Lönnberg E (1889) Bidrag till Kanendomen om i Sverge förs Kommande Cestoder. Bihang till Kongl Svanska vetenskaps-akademiens Handlingar 14: 1-69.

Luque JL, Cruces C, Chero J, Paschoal F, Alves PV, Da Silva AC, Sanchez L, Iannacone J (2016) Checklist of Metazoan parasites of fishes from Peru. Neotropical Helminthology 10: 301-375.

MacCallum GA (1921) Studies in helminthology. Zoopathologica 1: 137-284.

Maheswari JU, Lakshmi CV, Rao KH (1985) Studies on a new species of Acanthobothrium from Dasyatis uarnak (forskal) from Waltair coast. Rivista de Parassitologia 2: 39-44.

Maheswari JU, Sanaka S, Lakshmi CV, Rao KH (1987) Acanthobothrium waltairensis n. sp. (Cestoda: Tetraphyllidea) parasite of Dasyatis uarnak (Pisces: Condrichtehyes) from India. Revista Ibérica de Parasitología 47: 33-36.

Maleki L, Malek M, Palm HW (2013) Two new species of Acanthobothrium (Tetraphyllidea: Onchobothriidae) from Pastinachus cf. sephen (Myliobatiformes: Dasyatidae) from the Persian Gulf and Gulf of Oman. Folia Parasitologica 60: 448-456. https://doi.org/10.14411/ fp.2013.048

Maleki L, Malek M, Palm HW (2015) Four new species of Acanthobothrium van Beneden, 1850 (Cestoda: Onchoproteocephalidea) from the guitarfish, Rhynchobatus cf. djiddensis (Elasmobranchii: Rhynchobatidae), from the Persian Gulf and Gulf of Oman. Folia Parasitologica 62: 012 (011-015). https://doi.org/10.14411/fp.2015.012

Maleki L, Malek M, Palm HW (2019) Five new species of Acanthobothrium (Cestoda: Onchoproteocephalidea) from the long-tailed butterfly ray, Gymnura cf. poecilura (Elasmobranchii: Gymnuridae), from the Persian Gulf and Gulf of Oman. Zootaxa 4609: 289307. https://doi.org/10.11646/zootaxa.4609.2.5

Maleki L, Malek M, Rastgoo A (2018) Acanthobothrium chabahariensis n. sp. (Cestoda: Onchoproteocephalidea) in the cowtail stingray Pastinachus cf. sephen (Myliobatiformes: Dasyatidae) from the Gulf of Oman, Iran. Journal of Genetic Resources 4: 114-121.

Manger BR (1972) Some cestode parasites of the elasmobranchs Raja batis and Squalus acanthias from Iceland. Bulletin of the British Museum of Natural History (Zoology) 24: 161-181.

Marques F, Brooks DR, Barriga R (1997a) Six species of Acanthobothrium (Eucestoda: Tetraphyllidea) in stingrays (Chondrichthyes: Rajiformes: Myliobatoidei) from Ecuador. Journal of Parasitology 83: 475-484. https://doi.org/10.2307/3284414

Marques F, Brooks DR, Monks S (1995) Five new species of Acanthobothrium van Beneden, 1849 (Eucestoda: Tetraphyllidea: Onchobothriidae) in stingrays from the Gulf of Nicoya, Costa Rica. Journal of Parasitology 81: 942-951. https://doi.org/10.2307/3284046

Marques F, Centritto R, Stewart AS (1997b) Two new species of Acanthobothrium in Narcine entemedor (Rajiformes: Narcinidae) from the Northwest Coast of Guanacaste Peninsula, Costa Rica. Journal of Parasitology 83: 927-931. https://doi.org/10.2307/3284291 
Mayes MA, Brooks DR (1981) Cestode Parasites of Some Venezuelan Stingrays. Proccedings of the Biological Society of Washington 93: 1230-1238.

Mayes MA, Brooks DR, Thorson TB (1978) Two new species of Acanthobothrium Van Beneden 1849 (Cestoda: Tetraphyllidea) from freshwater stingrays in South Americana. Journal of Parasitology 64: 838-841. https://doi.org/10.2307/3279513

Merlo-Serna AI, Garcia-Prieto L (2016) A checklist of helminth parasites of Elasmobranchii in Mexico. ZooKeys 2016(563): 73-128. https://doi.org/10.3897/zookeys.563.6067

Monks S, Brooks DR, Pérez-Ponce de León G (1996) A new species of Acanthobothrium Van Beneden, 1849 (Eucestoda: Tetraphyllidea: Onchobothriidae) in Dasyatis longus Garman (Chondrichthyes: Myliobatiformes: Dasyatididae) from Chamela Bay, Jalisco, Mexico. Journal of Parasitology 82: 484-488. https://doi.org/10.2307/3284090

Monks S, Pulido-Flores G, Lara-Sánchez M (2015) Distribution extension of Acanthobothrium cartagenensis Brooks \& Mayes, 1980 (Tetraphyllidea: Onchobothriidae) in Urobatis jamaicensis (Cuvier, 1816) (Myliobatiformes: Urotrygonidae) from Quintana Roo, México. Check List 11: 1-3. https://doi.org/10.15560/11.4.1707

Ostrowski de Núñez M (1971) Estudios preliminares sobre la fauna parasitaria de algunos elasmobranquios del litoral bonaerense, Mar del Plata, Argentina. I. Cestodes y trematodes de Psammobatis microps (Günther) and Zapteryx brevirostris (Müller and Henle). Physis 30: 425-446.

Perrenoud N (1931) Recherches anatomiques et histologiques sur quelques cestodes de sélaciens. Revue Suisse de Zoologie 38: 469-555. https://doi.org/10.5962/bhl.part.117651

Rao V (1977) Acanthobothrium humantharaoi sp. n. (Cestoda: Tetraphyllidea, Oncobothriidae) from the nieuhof's eagle ray, Myliobatus nieuhofii (Bloch and Schneider) of Waltair coast, Bay of Bengal. Rivista di Parassitologia 38: 277-283.

Rees G, Williams HH (1965) The functional morphology of the scolex and the genetalia of Acanthobothrium coronotum (Rud.) (Cestoda: Tetraphyllidea). Parasitology 55: 617-651. https://doi.org/10.1017/S0031182000086212

Rêgo AA, Luna Dias AP (1976) Estudos de cestóides de peixes do Brasil. 3. Nota: cestóides de raias fluviais Paratrygonidae. Revista Brasileira de Biologia 36: 941-956.

Rêgo AA, Vicente JJ, Herrera NI (1968) Sôbre dois novos parásitos de peixe da costa do Peru (Cestoda, Tetraphyllidea). Memorias do Instituto Oswaldo Cruz 66: 145-149. https://doi. org/10.1590/S0074-02761968000200002

Reyda FB (2008) Intestinal helminths of freshwater stingrays in southeastern Peru, and a new genus and two new species of cestode. Journal of Parasitology 94: 684-699. https://doi. org/10.1645/GE-1230.1

Reyda FB, Caira JN (2006) Five New Species of Acanthobothrium (Cestoda: Tetraphyllidea) from Himantura uarnacoides (Myliobatiformes: Dasyatidae) in Malaysian Borneo. Comparative Parasitology 73: 49-71. https://doi.org/10.1654/4194.1

Riser NW (1955) Studies on cestode parasites of sharks and skates. Journal of the Tennessee Academy of Science 30: 265-311.

Robinson ES (1959) Some new cestodes from New Zealand marine fishes. Transactions of the Royal Society of New Zealand 86: 381-392. 
Robinson ES (1965) Cestoda (Tetraphyllidea and Trypanorhyncha) from marine fishes of New South Wales. Records of the Australian Museum 26: 341-348. https://doi.org/10.3853 /j.0067-1975.26.1965.683

Rodriguez TJ, Tantaleán-Vidaurre M (1980) Estudio sobre helmintos de peces elasmobranquios de la costa Peruana. 1. Nuevos registros de Tetraphyllideos. Boletin Peruano de Parasitologia 2: 71-75.

Rodríguez-Ibarra E, Pulido- Flores G, Violante González J, Monks S (2018) A new species of Acanthobothrium (Eucestoda: Onchobothriidae) in Aetobatus cf. narinari (Myliobatidae) from Campeche, México. Revista Brasileira de Parasitologia Veterinária 27: 66-73. https:// doi.org/10.1590/s1984-29612018009

Rudolphi CA (1810) Entozoorum, sive Vermium intestinalium historia naturalis. Treuttel et Würtz, 386 pp.

Rudolphi CA (1819) Entozoorum synopsis, cui accedunt mantissa duplex et indices locupletissimi. Humboldt-Universität, Rücker, 811 pp. https://doi.org/10.5962/bhl.title.9157

Rutledge KM (2019) A New Guitarfish of the Genus Pseudobatos (Batoidea: Rhinobatidae) with Key to the Guitarfishes of the Gulf of California. Copeia 107: 451-463. https://doi. org/10.1643/CI-18-166

Sanaka S, Vijaya Lakshmi C, Hanumantha Rao K (1993) Description of the new species Acanthobothrium satyanarayanaraoi from Rhinobatus granulatus from Waltair Coast, India. Boletin Chileno de Parasitologia 48: 15-17.

Sanaka S, Vijaya Lakshmi C, Hanumantha Rao K (1993) Description of the New species Acanthobothrium giganticum from Gymnura micrura from Waltair Coast. Rivista di Parassitologia 54: 15-17.

Schmidt GD (1973) Acanthobothrium urolophi sp. n., a tetraphyllidean cestode (Onchobothriidae) from an Australian stingaree. Proceedings of the Helminthological Society of Washington 40: 91-93.

Severino LR, Sarmiento BI (1979) Neuva especie del genero Acanthobothrium Van Benedem [sic] 1849; Cestode: Tetraphyllidea de Myliobatis peruvianus Garman 1913. Revista de Ciencias Universidad Nacional Mayor de San Marcos 71: 38-43.

Severino LR, Verano MR (1980) Acanthobothrium lusarmientoi n. sp. (Cestoda: Tetraphyllidea: Onchobothriidae) [de] Psammobatis caudispina Hildebrand, 1941 (Chondrichtyes: Rajidae) de Peru. Revista de Ciencias Universidad Nacional Mayor de San Marcos 72: 21-27. Shipley AE (1900) A description of the Entozoa collected by Dr Willey during his sojourn in the Western Pacific (Ed. A. Wiley, Cambridge University Press, UK.). Zoological Results 5: 531-568.

Southwell T (1912) A description of ten new species of cestode parasites from marine fishes of Ceylon, with notes on other cestodes from the same region. Colombo, Ceylon, Printed, London, 259-278.

Southwell T (1925) A monograph on the Tetraphyllidea with notes on related cestodes (Liverpool University Press, UK.). Memoirs of the Liverpool School of Tropical Medicine (New Series) 2: 1-368.

Southwell T (1930) Cestoda. Taylor and Francis, London, , 250-251. 
Srivastav AK, Capoor VN (1980) On Acanthobothrium dighaensis sp. n. (Onchobothriidae Braun, 1900) from Trygon marginatus. Helminthologia 17: 165-170.

Srivastav AK, Lohia S, Mathur N (1995) Acanthobothrium myliomaculata sp. nov. (Onchobohriidae, Cestoda) from the Myliobates maculata from Madras (India). Flora and Fauna 1: 43-45.

Subhapradha CK (1955) Cestode parasites of fishes of Madras coast. Indian Journal of Helminthology 7: 41-132.

Tantaleán-Vidaurre M (1991) Nuevos helmintos parasitos en peces elasmobranquio de la costa peruana. Boletin de Lima 73: 25-28.

Tazerouti F, Kechemir-Issad N, Euzet L (2009) Acanthobothrium minus n. sp. (Tetraphyllidea: Onchobotriidae) parasite de Raja asterias (Elasmobranchii : Rajidae) en Méditerranée. Parasite 16: 203-207.

Twohig ME, Caira JN, Fyler CA (2008) Two new cestode species from the dwarf whipray, Himantura walga (Batoidea: Dasyatidae), from Borneo, with comments on site and mode of attachment. Journal of Parasitology 94: 1118-1127. https://doi.org/10.1645/GE-1475.1

van Beneden PJ (1850) Recherches sur la faune littorale de Belgique. Les vers cestoides, considérés sous le rapport physiologique, embryogénique et zooclassique. Mémoires de l'Academie Royale des Sciences, des Lettres et des Beaux-Arts de Belgique 25: 1-204. https://doi.org/10.5962/bhl.title.47103

Vardo-Zalik AM, Campbell RA (2011) Five new species of Acanthobothrium van Beneden, 1849 (Cestoda: Tetraphyllidea) in elasmobranchs from the northwest Atlantic and Gulf of Mexico with the first records from smooth-hound sharks and guitarfish. Zootaxa 2828: 41-64. https://doi.org/10.11646/zootaxa.2838.1.3

Vaz DFB, Carvalho MRd (2018) New Species of Squatina (Squatiniformes: Squatinidae) from Brazil, with Comments on the Taxonomy of Angel Sharks from the Central and Northwestern Atlantic. Copeia 106: 144-160. https://doi.org/10.1643/CI-17-606

Verma S-C (1928) Some Cestodes from Indian fishes including four new species of Tetraphyllidea and revised keys to the genera Acanthobothrium and Gangesia. Allahabad University Studies 4: 119-176.

Wang P-Q (1984) Notes on some cestodes of fishes in Fujian province, with a list of fish cestodes recorded from China. Wuyi Science Journal 4: 71-83.

Wang Y-H, Yang W-C (2001) Two New Species of Acanthobothrium from Marine Fishes in Xiaroen, Fujian, China (Cestoda: Tetraphyllidea: Onchobothridae). Journal of Xiamen University (Natural Science) 40: 943-948.

Williams HH (1960) A list of parasitic worms, including 22 new records, from marine fishes caught off the British Isles. Annals and Magazine of Natural History 2: 705-715. https:// doi.org/10.1080/00222935908655756

Williams HH (1962) Acanthobothrium sp. nov. (Cestoda: Tetraphyllidea) and a comment on the order Biporophyllaeidea. Parasitology 52: 67-76. https://doi.org/10.1017/ S0031182000024008

Williams HH (1968) Acanthobothrium quadripartitum sp. nov. (Cestoda: Tetraphyllidea) from Raja naevus in the North Sea and English Channel. Parasitology 58: 105-110. https://doi. org/10.1017/S0031182000073467 
Williams HH (1969) The genus Acanthobothrium van Beneden 1849 (Cestoda: Tetraphyllidea). Nytt Magasin for Zoologi 17: 1-56.

Yamaguti S (1934) Studies on the helminth fauna of Japan. Part 4. Cestodes of fishes. Japanese Journal of Zoology 6: 1-112.

Yamaguti S (1952) Studies on the helminth fauna of Japan. Part 49. Cestodes of fishes, II. Acta Medica Okayama 8: 1-97.

Yamaguti S (1959a) The cestodes of vertebrates. Wiley Interscience Publications, New York.

Yamaguti S (1959b) Systema Helminthum: Cestoda. Wiley Interscience Publications, New York, 860 pp.

Yang C, Sun Y, Zhi T, Iwaki T, Reyda FB, Yang T (2016) Two new and one redescribed species of Acanthobothrium (Cestoda: Onchoproteocephalidea: Onchobothriidae) from Dasyatis akajei (Myliobatiformes: Dasyatidae) in the China Sea. Zootaxa 4169: 286-300. https:// doi.org/10.11646/zootaxa.4169.2.3

Yang WLY (1994) Two New Species of Acanthobothrium Cestodes (Tetraphyllidea: Onchobothriidae) from Saltwater Fishes in Xiamen.South Fujian.China. Journal of Xiamen University (Natural Science) 33: 532-536.

Yokota L, Carvalho MRd (2017) Taxonomic and morphological revision of butterfly rays of the Gymnura micrura (Bloch \&amp; Schneider 1801) species complex, with the description of two new species (Myliobatiformes: Gymnuridae). 2017 4332: 1-74. https://doi. org/10.11646/zootaxa.4332.1.1

Yoshida S (1917) Some cestodes from Japanese selachians including five new species. Parasitology 9: 560-592. https://doi.org/10.1017/S003118200000620X

Young RT (1954) Cestodes of sharks and rays in Southern California. Proceedings of the Helminthological Society of Washington 21: 106-112.

Zamparo D, Brooks DR, Hoberg EP, McLennan DA (2001) Phylogenetic analysis of the Rhabdocoela (Platyhelminthes) with emphasis on the Neodermata and their relatives. Zoologica Scripta 30: 59-77. https://doi.org/10.1046/j.1463-6409.2001.00050.x

Zaragoza-Tapia F, Pulido-Flores G, Violante-González J, Monks S (2019) Two new species of Acanthobothrium Blanchard, 1848 (Onchobothriidae) in Narcine entemedor Jordan \& Starks, 1895 (Narcinidae) from Acapulco, Guerrero, Mexico. ZooKeys 852: 1-21. https:// doi.org/10.3897/zookeys.852.28964

Zaragoza-Tapia F, Pulido-Flores G, Monks S (2020) Three new species of Acanthobothrium Blanchard, 1848 (Cestoda: Onchoproteocephalidea) in Stingrays (Dasyatidae) from the Pacific coast in Mexico. Zootaxa 4766 (1): 139-172. https://doi.org/10.11646/zootaxa.4766.1.8

Zschoche M, Caira JN, Fyler CA (2011) A new species of Acanthobothrium van Beneden, 1850 (Tetraphyllidea: Onchobothriidae) from Pastinachus atrus (Macleay) (Batoidea: Dasyatidae) in Australian waters, with a reassessment of the host associations of Acanthobothrium spp. parasitising Pastinachus spp. Systematic Parasitology 78: 109-118. https://doi. org/10.1007/s11230-010-9279-2

Zschokke F (1888) Recherches sur la structure anatomique et histologique des Cestodes. Institut national genevois. 